\title{
Towards incorporating asexual fungi in a natural classification: checklist and notes 2012-2016
}

\author{
Wijayawardene $\mathrm{NN}^{1,2}$, Hyde $\mathrm{KD}^{2}$, Tibpromma $\mathrm{S}^{2}$, Wanasinghe $\mathrm{DN}^{2}$, \\ Thambugala $\mathrm{KM}^{2}$, Tian $\mathbf{Q}^{2}$, Wang $\mathbf{Y}^{3}$, Fu $\mathbf{L}^{1}$ \\ ${ }^{1}$ Shandong Institute of Pomologe, Taian, Shandong Province, 271000, China \\ ${ }^{2}$ Center of Excellence in Fungal Research, Mae Fah Luang University, Chiang Rai, 57100, Thailand \\ ${ }^{3}$ Department of Plant Pathology, Agriculture College, Guizhou University, Guiyang 550025, People's Republic of \\ China
}

Wijayawardene NN, Hyde KD, Tibpromma S, Wanasinghe DN, Thambugala KM, Tian Q, Wang Y 2017 - Towards incorporating asexual fungi in a natural classification: checklist and notes 20122016. Mycosphere 8(9), 1457-1555, Doi 10.5943/mycosphere/8/9/10

\begin{abstract}
Incorporating asexual genera in a natural classification system and proposing one name for pleomorphic genera are important topics in the current era of mycology. Recently, several polyphyletic genera have been restricted to a single family, linked with a single sexual morph or spilt into several unrelated genera. Thus, updating existing data bases and check lists is essential to stay abreast of these recent advanes. In this paper, we update the existing outline of asexual genera and provide taxonomic notes for asexual genera which have been introduced since 2012 . Approximately, 320 genera have been reported or linked with a sexual morph, but most genera lack sexual morphs.
\end{abstract}

Keywords - Article 59.1 - Coelomycetous - Hyphomycetous - One name - Pleomorphism

\section{Introduction}

The recent outlines and monographs of different taxonomic groups (including artificial groups i.e. coelomycetes and hyphomycetes) tried to incorporate asexual genera in a natural classification system (Hyde et al. 2013, Maharachchikumbura et al. 2015, 2016, Senanayake et al. 2015, Wijayawardene et al. 2016, 2017). However, many asexual genera still lack sequence data and thus their placements have not been determined. These fungi are treated as Ascomycota, genera incertae sedis. Nevertheless, several recent studies have re-collected historic genera, subjected then to morpho-molecular analyses and provided taxonomic placements (Dai et al. 2014, Wijayawardene et al. 2013, 2016, Crous et al. 2015a).

Seifert et al. (2011), Hyde et al. (2013), Johnston et al. (2014), Wijayawardene et al. (2014, 2017), Maharachchikumbura et al. (2015, 2016), Rosman et al. (2013, 2015a, b, 2016), Réblová et al. $(2015,2016)$ and Senanayake et al. (2015) have provided significant taxonomic and nomenclatural changes for different taxonomic groups based on DNA sequence analyses.

In this article, we incorporate asexual genera in a natural classification and update existing outlines i.e. Hyde et al. (2011) and Wijayawardene et al. (2012). 
The outline of classification system follows Lumbsch \& Huhndorf (2010) as a starting point. The generic names are extracted from Kirk et al. (2008, 2013), Index Fungorum (2017) and research articles since 2011 to end of 2016. Recent name changes of pleomorphic genera (according to Article 59.1) are also incorporated based on recent articles as in Table 1.

The present article lists asexual genera or '-like' genera under the appropriate fungal classification and the link to a sexual genus given where found. Dubious genera are excluded, but can be added if they are later found to be good genera. Fossil fungi are also excluded as it would be impossible with present techniques available to provide a natural classification for them. A question mark before the entry means that its placement in that family or order is not fully established and generally follows Lumbsch \& Huhndorf (2010). A question mark before asexual morph means it is not clear that this is the asexual morph of this genus, although there are many cases without a question mark where the link has not equivocally been proven. An asterisk following the names indicates an entry in the "Notes" section.

We have added $(\mathrm{H})=$ hyphomycete, $(\mathrm{C})=$ coelomycete,$(\mathrm{Y})=$ yeast and $(\mathrm{R})=$ Rust against entries where known. The asexual genera which are reported with or linked with sexual morphs are also included to the outline and indicated by \#.

Table 1. Articles used to update the name changes of pleomorphic genera

\begin{tabular}{ll}
\hline Taxonomic group & Reference \\
\hline Ascomycota & Wijayawardene et al. 2017 \\
Diaporthales & Rossman et al. 2013, 2015b \\
Dothideomycetes & Wijayawardene et al. 2014, Rossman et al. \\
& 2015a \\
Leotiomycetes & Johnston et al. 2014 \\
Magnaporthales & Zhang et al. 2016 \\
Orbiliaceae (Orbiliomycetes) & Baral et al. 2016, Ekanayaka et al. 2017 \\
Sordariomycetes & Maharachchikumbura et al. 2015, 2016, \\
& Réblová et al. 2016a \\
Xylariales & Wendt et al. 2017 \\
Xylariomycetidae & Senanayake et al. 2015 \\
Pezizomycetes & Healy et al. 2016 \\
\hline
\end{tabular}

\section{Index to Ascomycota}

Phylum ASCOMYCOTA Caval-Sm.

Subphylum TAPHRINOMYCOTINA O.E. Erikss. \& Winka

Class Taphrinomycetes O.E. Erikss. \& Winka

Taphrinales Gäum. \& C.W. Dodge

Protomycetaceae Gray

Saitoella Goto et al. 1987 (Y)

\section{Taphrinaceae Gäum.}

Lalaria R.T. Moore 1990 (Y)

Subphylum SACHAROMYCOTINA O.E. Erikss. \& Winka

Class Saccharomycetes O.E. Erikss. \& Winka

Saccharomycetales Kudrjanzev

Dipodascaceae Engl. \& E. Gilg

Blastobotrys Klopotek 1967 (H)
Geotrichum Link 1809 (H)

Saprochaete Coker \& Shanor 1939 (H)

Lipomycetaceae E.K. Novák \& Zsolt

Myxozyma Van der Walt et al. 1981 (Y)

Pichiaceae Zender

Brettanomyces N.H. Claussen ex Custers 1940 (Y)

(Y)

Hyphopichia Arx \& Van der Walt 1976

Saccharomycodaceae Kudrjanzev

Kloeckera Janke 1923 (Y)

Trigonopsidaceae M.A. Lachance \& C.P. Kurtzman

Botryozyma Shann \& M.T. Sm. 1992 (Y)

Trigonopsis Schachner 1929 (Y) 
Saccharomycetales genera incertae sedis Aciculoconidium D.S. King \& S.C. Jong 1976 (Y)

Candida Berkhout 1923 (Y)

Macrorhabdus Tomasz. et al. 2003 (Y)

Subphylum PEZIZOMYCOTINA O.E. Erikss. \& Winka

Class Arthoniomycetes O.E. Erikss. \& Winka Arthoniales Henssen ex D. Hawksw. \& O.E. Erikss.

Arthoniaceae Reichenb. ex Reichenb.

Briancoppinsia Diederich et al. 2012 (C)*

Helicobolomyces Matzer 1995 (C)

Reichlingia Diederich \& Scheid. 1996 (H)

Tylophoron Nyl. ex Stizenb. $1862(\mathbf{H})^{\# *}$

Lichenostigmatales Ertz et al.

Phaeococcomycetaceae McGinnis \& Schell Etayoa Diederich \& Ertz 2014 (C) ${ }^{\# *}$ Phaeococcomyces de Hoog 1979 (H)

Class Dothideomycetes sensu O.E. Erikss. \& Winka

Subclass Dothideomycetidae P.M. Kirk et al.

Capnodiales Woron.

Antennulariellaceae Woron.

Antennariella Bat. \& Cif. 1963 (C)

Capnodiaceae (Sacc.) Höhn. ex Theiss.

Capnodium Mont. 1848 (C)

Fumiglobus D.R. Reynolds \& G.S. Gilbert $2006(\mathbf{C}) *$

Leptoxyphium Speg. 1918 (H)

Phragmocapnias Theiss. \& Syd. 1918 (C) ${ }^{\#}$

Cladosporiaceae Nann.

(H)

Acroconidiella J.C. Lindq. \& Alippi 1964

Cladosporium Link $1816(\mathbf{H})^{\#}$

Graphiopsis Trail 1889 (H)

Hoornsmania Crous 2007 (H)

Rachicladosporium Crous et al. 2007 (H)

Toxicocladosporium Crous \& U. Braun 2007 (H)

Verrucocladosporium K. Schub. et al. 2007 (H)

Dissoconiaceae Crous \& de Hoog

Dissoconium de Hoog et al. 1983 (H)

(H)*
Ramichloridium ex de Hoog 1977 (H)

Uwebraunia Crous \& M.J. Wingf. 1996 (H)

Euantennariaceae Hughes \& Corlett*

Capnokyma S. Hughes 1975 (H)

Hormisciomyces Bat. \& Nascim. 1957 (H)

Plokamidomyces Bat. et al. 1957 (H)

Racodium Fr. 1829 (H)

Trichothallus F. Stevens 1925 (H)

Extremaceae Quaedvl. \& Crous

Extremus Quaedvl. \& Crous 2014 (H)*

Petrophila de Hoog \& Quaedvl. 2014 (H)*

Pseudoramichloridium Cheew. \& Crous 2009 (H)

Saxophila Selbmann \& de Hoog 2015 (H)*

Staninwardia B. Sutton 1971 (C)

Vermiconia Egidi \& Onofri 2014 (H)*

Metacapnodiaceae S. Hughes \& Corlett

Capnobotrys S. Hughes 1970 (H)

Capnophialophora S. Hughes 1966 (H)

Metacapnodium Speg. 1918 (H)

Mycosphaerellaceae Lindau

Acervuloseptoria Crous \& Jol. Roux 2014

(C)*

Acrodontium de Hoog 1972 (H)

Annellosympodiella Crous \& Assefa 2014 (H)*

Apseudocercosporella Videira \& Crous 2016 (H)*

Asperisporium Maubl. 1913 (H)

Brunneosphaerella Crous 2009 (C)

Camptomeriphila Crous \& M.J. Wingf. $2016(\mathbf{C})^{*}$

Caryophylloseptoria Verkley et al. 2013

(C)*

Cercospora Fresen. $1863(\mathbf{H})^{\#_{*}}$

Cercosporella Sacc. 1880 (H)

Colletogloeum Petr. 1953 (C)

Cytostagonospora Bubák 1916 (C)*

Davisoniella H.J. Swart 1988 (C)

Dictyosporina Abreu et al. 2017 (H)*

Distocercospora N. Pons \& B. Sutton 1988

(H)

Dothistroma Hulbary 1941 (C)

$(\mathbf{H})^{\# *}$

Epicoleosporium Videira \& Crous 2016

Filiella Videira \& Crous 2016 (H)*

Fusoidiella Videira \& Crous 2016 (H)* 
Geastrumia Bat. 1960 (C)*

Janetia M.B. Ellis 1976 (H)*

Lecanosticta Syd. 1922 (C)

Microcyclosporella Jana Frank et al. 2010

(H)

$(\mathbf{H})^{\# *}$

Miuraea Hara 1948 (H)

Mycosphaerelloides Videira \& Crous 2016

Mycovellosiella Rangel 1918 (H)

Neoceratosperma Crous \& Cheew. 2014 (H)*

Neocercospora M. Bakhshi et al. 2015 (H)*

Neodeightoniella Crous \& W.J. Swart 2013 (H)* (H)*

Neopenidiella Quaedvl. \& Crous 2014

Neopseudocercospora Crous 2013 (H)*

Neopseudocercosporella Videira \& Crous $2016(\mathbf{H})^{\# *}$

Neoramichloridium Phook. et al. 2017 (H)*

Neoseptoria Quaedvl. et al. 2013 (C)*

Pallidocercospora Crous $2012(\mathbf{H})^{\# *}$

Pantospora Cif. 1938 (H)

Paracercospora Deighton 1979 (H)

Parastenella J.C. David 1991 (H)

Passalora Fr. 1849 (H)

Periconiella Sacc. 1885 (H)

Phaeocercospora Crous 2012 (H)

Phaeophleospora Rangel 1916 (C)

Phaeothecoidea Crous 2007 (H)

Phloeospora Wallr. 1833 (C)

(C)*

Polyphialoseptoria Quaedvl. et al. 2013

Polythrincium Kunze $1817(\mathbf{H})^{\# *}$

Protostegia Cooke 1880 (C)*

Pseudocercospora Speg. 1910 (H)

Pseudocercosporella Deighton 1973 (H)

Pseudocercosporidium Deighton 1973 (H)

Ramularia Unger 1833 (H)

Ramulispora Miura 1920 (H)

Ruptoseptoria Quaedvl. et al. 2013 (C)*

Scolecostigmina U. Braun 1999 (H)

Septoria Sacc. 1884 (C)

Sonderhenia H.J. Swart \& J. Walker 1988

(C)

Sphaerulina Sacc. 1878 (H)

Stromatoseptoria Quaedvl. et al. 2013

(C)*

Trochophora R.T. Moore 1955 (H)

Uwemyces Hern.-Restr. et al. 2016 (H)*
Xenoramularia Videira et al. 2016 (H)*

Zasmidium Fr. 1849 (H)

Zymoseptoria Quaedvl. \& Crous 2011 (C)

Neodevriesiaceae Quaedvl. \& Crous

$(\mathbf{H})^{\# *}$

Neodevriesia Quaedvl. \& Crous 2014

Tripospermum Speg. 1918 (H)

Phaeothecoidiellaceae K.D. Hyde \& Hongsanan*

Houjia G.Y. Sun \& Crous 2010 (H)

Phaeothecoidiella Batzer \& Crous 2010

(H)

Teratosphaeriaceae Crous \& U. Braun

Acidomyces B.J. Baker et al. 2008 (H)

Acrodontium de Hoog 1972 (H)

Apenidiella Quaedvl. \& Crous 2014 (H)*

Batcheloromyces Marasas et al. 1975 (H)

Baudoinia J.A. Scott \& Unter. 2007 (H)

Bryochiton Döbbeler \& Poelt $1978(\mathbf{H})^{\# *}$

Camarosporula Petr. 1954 (C) ${ }^{\#}$

Capnobotryella Sugiy. 1987 (H)

(H)*

Catenulomyces Egidi \& de Hoog 2014

Catenulostroma Crous \& U. Braun 2007

(H)

Constantinomyces Egidi \& Onofri 2014

(H)*

Devriesia Seifert \& N.L. Nick. 2004 (H)

Elasticomyces Zucconi \& Selbmann 2008

(H)

Eupenidiella Quaedvl. \& Crous 2014 (H)*

Fodinomyces Vázquez-Campos \& Neilan $2014(\mathbf{H})^{*}$

Friedmanniomyces Onofri 1999 (H)

Hispidoconidioma Tsuneda \& Davey 2010

(H)

Hortaea Nishim. \& Miyaji 1984 (H)

Hyweljonesia R.G. Shivas et al. 2016 (H)*

Incertomyces Egidi \& Zucconi 2014 (H)*

(H)*

Lapidomyces de Hoog \& Stielow 2014

Leptomelanconium Petr. 1923 (C)

Meristemomyces Isola \& Onofri 2014 (H)*

Microcyclospora J. Frank et al. 2010 (H)

Monticola Selbmann \& Egidi 2014 (H)*

Myrtapenidiella Quaedvl. \& Crous 2014

(H)*

Neocatenulostroma Quaedvl. \& Crous $2014(\mathbf{H})^{*}$ 
Neophaeothecoidea Quaedvl. \& Crous $2014(\mathbf{H})^{*}$

Neotrimmatostroma Quaedvl. \& Crous $2014(\mathbf{H})^{*}$

Oleoguttula Selbmann \& de Hoog 2014 (H)*

Parapenidiella Crous \& Summerell 2012 (H)

Penidiella Crous \& U. Braun 2007 (H)

Penidiellopsis Sandoval-Denis et al. 2016 (H)*

Phaeothecoidea Crous 2007 (H)

Pseudotaeniolina J.L. Crane \& Schokn. 1986 (H)

Queenslandipenidiella Quaedvl. \& Crous 2014 (H)*

Ramopenidiella Crous \& R.G. Shivas 2014

(H)*

Readeriella Syd. \& P. Syd. 1908 (C)

Recurvomyces Selbmann \& de Hoog 2008 (H)

Stenella Syd. 1930 (H)

Teratoramularia Videira et al. 2016 (H)*

Teratosphaeria Syd. \& P. Syd. 1912 (C)

Xenoconiothyrium Crous \& Marinc. 2011

(C) (H)*

Xenopenidiella Quaedvl. \& Crous 2014

Capnodiales, genera incertae sedis

Arthrocatena E. Egidi \& L. Selbmann 2014 (H)*

Eriosporella Höhn. 1916 (C)*

Hyphoconis Egidi \& Quaedvl. 2014 (H)*

Neohortaea Quaedvl. \& Crous 2014 (H)*

Phaeotheca Sigler et al. 1981 (H)

Pseudoepicoccum M.B. Ellis 1971 (H)

Ramimonilia B. Stielow. \& Quaedvl. 2014

(H)*

Readerielliopsis Crous \& Decock 2015 (C)*

Septocyta Petr. 1927 (C)

Xenosonderhenia Crous 2012 (C)*

Dothideales Lindau

Dothideaceae Chevall.

Dothiora Fr. 1849 (C)

Endoconidioma Tsuneda et al. 2004 (C)

Hormonema Lagerb. \& Melin 1927 (H)

Kabatina R. Schneid. \& Arx 1966 (C)

Neocylindroseptoria Thambug. \& K.D. Hyde 2014 (C)*
$(\mathbf{C})^{\# *}$

Neophaeocryptopus Wanas. et al. 2016

Rhizosphaera L. Mangin \& Har. 1907 (C)

Saccotheciaceae Bonord.

(H)

Aureobasidium Viala \& G. Boyer 1891

Kabatiella Bubák 1907 (H)

Pseudoseptoria Speg. 1910 (C)

Saccothecium Fr. $1836(\text { C })^{\# *}$

Selenophoma Maire 1907 (C)

Dothideales, genera incertae sedis

Celosporium Tsuneda \& Davey 2010 (H)

Coniozyma Crous 2008 (C)

Myriangiales Starbäck

Elsinoaceae Höhn. ex Sacc. \& Trotter

Elsinö̈ Racib. 1900 (C)

Subclass Pleosporomycetidae C.L. Schoch et al.

Hysteriales Lindau

Hysteriaceae Chevall.

Coniosporium Link 1809 (H)

Gloniopsis De Not. 1847 with aposphaeria-

like asexual morph (C) ${ }^{\#}$

Hysterobrevium E. Boehm \& C.L. Schoch

2010 with aposphaeria-like asexual morph (C) ${ }^{\#}$

Rhytidhysteron Speg. 1881 (C)*

Mytilinidiales Boehm et al.

Gloniaceae (Corda) Boehm et al.

Cenococcum Moug. \& Fr. 1829 (C)

Purpurepithecium Jayasiri \& K.D. Hyde 2017

$(\mathbf{H})^{\# *}$

Mytilinidiaceae Kirschst.

Septonema Corda 1837 (H)

Taeniolella S. Hughes 1958 (H)

Pleosporales Luttrell ex M.E. Barr

Acrocalymmaceae Crous \& Trakun.

Acrocalymma Alcorn \& J.A.G. Irwin 1987

(C)

Amniculicolaceae. Zhang et al.

Amniculicola Y. Zhang ter \& K.D. Hyde $2008(\mathbf{H})^{\#}$

Murispora Y. Zhang bis et al. 2009 (C) ${ }^{\# *}$

Vargamyces Tóth 1980 (H) 
Amorosiaceae Thambug. \& K.D. Hyde

Amorosia Mantle \& D. Hawksw. 2006 (H) $(\mathbf{H})^{\# *}$

Angustimassarina Thambug. et al. 2015

Aquasubmersaceae A. Hashim. \& Kaz. Tanaka $(\mathbf{C})^{\# *}$

Aquasubmersa K.D. Hyde \& Huang Zhang

Bambusicolaceae D.Q. Dai \& K.D. Hyde $(\mathbf{C})^{\# *}$

Bambusicola D.Q. Dai \& K.D. Hyde 2012 $(\mathbf{C})^{\# *}$

Palmiascoma Phook. \& K.D. Hyde 2015

Biatriosporaceae K.D. Hyde

$(\mathbf{C})^{\#}$

Biatriospora K.D. Hyde \& Borse 1986

Camarosporiaceae Wanas. et al.

Camarosporium Schulzer 1870 (C) ${ }^{\#}$

Camarosporomyces Crous 2017 (C)*

Coniothyriaceae W.B. Cooke

Coniothyrium Corda 1840 (C)

Staurosphaeria Rabenh. 1858 (C) ${ }^{\# *}$

Corynesporascaceae Sivan.

Corynespora Güssow 1906 (H)

Cryptocoryneaceae A. Hashim. \& Kaz. Tanaka Cryptocoryneum Fuckel 1870 (H)*

Cucurbitariaceae G. Winter

Cucurbitaria Gray 1821 (C) ${ }^{\# *}$

Neocucurbitaria Wanas. et al. 2017 (C) ${ }^{\# *}$

Pyrenochaeta De Not. 1849 (C)

Pyrenochaetopsis Gruyter et al. 2010 (C)

Syncarpella Theiss. \& Syd. 1915 (C) ${ }^{\# *}$

Cyclothyriellaceae Jaklitsch \& Voglmayr

$(\mathbf{C})^{\# *}$

Cyclothyriella Jaklitsch \& Voglmayr 2016

Dacampiaceae Körb.

Munkovalsaria Aptroot 1995 (H) ${ }^{\# *}$

Dictyosporiaceae Boonmee \& K.D. Hyde

(H)

Cheirosporium L. Cai \& K.D. Hyde 2008

Dendryphiella Bubák \& Ranoj. 1914 (H)*
(H)*

Dictyocheirospora D’souza et al. 2016

Dictyopalmispora Pinruan et al. 2016 (H)*

Dictyosporium Corda $1836(\mathbf{H})^{*}$

Digitodesmium P.M. Kirk 1981 (H)*

Jalapriya D'souza et al 2016 (H)*

Pseudocoleophoma Kaz. Tanaka \& K. Hiray. 2015 (C) ${ }^{\# *}$

Pseudodictyosporium Matsush. 1971 (H)

Vikalpa D'souza et al 2016 (H)*

Didymellaceae Gruyter et al.

Allophoma Q. Chen \& L. Cai 2015 (C)*

Ascochyta Lib. 1830 (C) ${ }^{\# *}$

Boeremia Aveskamp et al. 2010 (C) ${ }^{\#_{*}}$

Calophoma Q. Chen \& L. Cai 2015 (C)*

Chaetasbolisia Speg. 1918 (C)

Dactuliochaeta G.L. Hartm. \& J.B. Sinclair 1988 (C)

Didymellocamarosporium Wijayaw. \& K.D. Hyde 2016 (C)*

(H)*\#

Didysimulans Tibpromma et al. 2017

Endocoryneum Petr. 1922 (C)*

Epicoccum Link 1815 (H)

Heterophoma Q. Chen \& L. Cai 2015 (C)*

Leptosphaerulina McAlpine 1902 (H)

$(\mathbf{C})^{\# *}$

Neoascochyta Q. Chen \& L. Cai 2015 $(\mathrm{C})^{\# *}$

Neodidymelliopsis Q. Chen \& L. Cai 2015

Neomicrosphaeropsis Thambug. et al. $2016(\mathbf{C})^{*}$

Nothophoma Q. Chen \& L. Cai 2015 (C)* $(\mathbf{C})^{\# *}$

Paraboeremia Q. Chen \& L. Cai 2015

Peyronellaea Gold. ex Togliani 1952 (C)

Phaeomycocentrospora Crous et al. 2012

(H)*

Phoma Sacc. 1880 (C)

Phomatodes Q. Chen \& L. Cai 2015 (C)*

Platychora Petr. 1925 (C) \#* $^{\# *}$

Pseudoascochyta Valenzuela-Lopez et al. $2016(\mathbf{C})^{*}$

Pseudohendersonia Crous \& M.E. Palm 1999 (C)*

Stagonosporopsis Died. $1912(\mathbf{C})^{\# *}$ $(\mathbf{C})^{\# *}$

Xenodidymella Q. Chen \& L. Cai 2015

Didymosphaeriaceae Munk

Alloconiothyrium Verkley et al. 2014 (C)* 
Austropleospora R.G. Shivas \& L. Morin $2010(\mathbf{C})^{\# *}$

Kalmusia Niessl $1872(\mathbf{C})^{\# *}$

(C)*

Paracamarosporium Wijayaw. et al. 2014

Paraconiothyrium Verkley 2004 (C)

Paraphaeosphaeria O.E. Erikss. 1967 (C)

Pseudocamarosporium Wijayaw. \& K.D. Hyde 2014 (C)*

Pseudopithomyces Ariyaw. \& K.D. Hyde 2015 (H)*

Verrucoconiothyrium Crous 2015 (C)*

Xenocamarosporium Crous \& M.J. Wingf. $2015(\mathbf{C})^{*}$

Fuscostagonosporaceae Jayasiri et al.*

Fuscostagonospora Kaz. Tanaka \& K. Hiray. 2015 (C)*

Hermatomycetaceae Locq. ex A. Hashim. \& Kaz. Tanaka

Hermatomyces Speg. 1910 (H)*

Hypsostromataceae Huhndorf

Hypsostroma Huhndorf $(\mathbf{C})^{\# *}$

\section{Latoruaceae Crous}

Latorua Crous 2015 (H)*

Matsushimamyces Rahul Sharma \& Rohit Sharma 2015 (H)*

Pseudoasteromassaria M. Matsum. \& Kaz. Tanaka $2016(\mathbf{C})^{\# *}$

Lentitheciaceae Yin. Zhang et al.

Lentithecium K.D. Hyde et al. 2009 (C) ${ }^{\# *}$ Murilentithecium Wanas. et al. 2014 (C) ${ }^{\# *}$

Phragmocamarosporium Wijayaw. et al. 2015 (C)*

Pleurophoma Höhn. 1914 (C)*

Setoseptoria Quaedvl. 2013 (C)*

Towyspora Wanas. et al. $2016(\mathbf{C})^{\# *}$

Leptosphaeriaceae M.E. Barr

Acicuseptoria Quaedvl. et al. 2013 (C)*

Alternariaster E.G. Simmons 2007 (H)*

Chaetoplea (Sacc.) Clem. 1931 (C)

Heterosporicola Crous 2017 (C)*

Leptosphaeria Ces. \& De Not. $1863(\text { C })^{\#_{*}}$

$(\mathbf{C})^{\# *}$

Paraleptosphaeria Gruyter et al. 2012

Plenodomus Preuss 1851 (C)*

Querciphoma Crous 2017 (C)*
Sphaerellopsis Cooke 1883 (C)

Subplenodomus Gruyter et al. 2012 (C)*

Libertasomycetaceae Crous*

Libertasomyces Crous \& Roets 2016 (C)*

Neoplatysporoides Crous \& M.J. Wingf. $2015(\mathbf{C})^{\# *}$

Lindgomycetaceae K. Hiray. et al.

Clohesyomyces K.D. Hyde 1993 (C)

Lolia Abdel-Aziz \& Abdel-Wahab 2010

(C)

Longipedicellataceae Phukhams. et al.

Longipedicellata H. Zhang et al. 2016 (H)

Lophiostomataceae Sacc.

Coelodictyosporium Thambug. \& K.D. Hyde $2015(\mathbf{C})^{\# *}$

Dimorphiopsis Crous 2013 (C)*

Lophiostoma Ces. \& De Not. 1863 (C) ${ }^{\# *}$

Lophiotremataceae K. Hiray. \& Kaz. Tanaka $(\mathbf{C})^{\# *}$

Atrocalyx A. Hashim. \& Kaz. Tanaka 2017

Crassimassarina A. Hashim. \& Kaz. Tanaka $2017(\mathbf{C})^{\# *}$

Cryptoclypeus A. Hashim. \& Kaz. Tanaka $2017(\mathbf{C})^{\# *}$

Galeaticarpa A. Hashim. \& Kaz. Tanaka $2017(\mathbf{C})^{\# *}$

Pseudocryptoclypeus A. Hashim. \& Kaz. Tanaka 2017 (C) $)^{\# *}$

Macrodiplodiopsidaceae Voglmayr et al.

Macrodiplodiopsis Petr. 1922 (C) ${ }^{\# *}$

Pseudomonodictys Doilom et al. 2015

(H)*

Massarinaceae Munk

Helminthosporiella Hern.-Restr. et al. 2016 (H)*

Helminthosporium Link $1809(\mathbf{H})^{\# *}$

Pseudodidymosphaeria Thambug. \& K.D. Hyde 2015 (C) ${ }^{\# *}$

Pseudosplanchnonema Chethana et al. $2015(\mathbf{C})^{\# *}$

Stagonospora (Sacc.) Sacc. 1884 (C)

Suttonomyces Wijayaw. et al. 2015 (C)*

Melanommataceae G. Winter 
Aposphaeria Sacc. 1880 (C)*

Beverwykella Tubaki 1975 (H)

Exosporiella P. Karst. 1892 (H)

Fusiconidium J.F. Li et al. 2017 (H)*

Melanocamarosporium Wijayaw. et al. $2016(\mathbf{C})^{*}$

$\#_{*}$

Melanomma Nitschke ex Fuckel 1870 (C)

Phragmocephala E.W. Mason \& S. Hughes 1951 (H)

Pleotrichocladium Hern.-Restr. et al. 2017 (H)*

Pseudotrichia Kirschst. 1939 (C) ${ }^{\# *}$

Seifertia Partr. \& Morgan-Jones 2002 (H)*

Sporidesmiella P.M. Kirk 1982 (H)

Microsphaeropsidaceae Q. Chen et al.

Microsphaeropsis Höhn. 1917 (C)*

Neocamarosporiaceae Wanas. et al.*

Neocamarosporium Crous \& M.J. Wingf. $2014(\mathbf{C})^{*}$

Neohendersoniaceae Giraldo \& Crous

Neohendersonia Petr. 1921 (C)

Neophaeosphaeriaceae Ariyaw. et al.

Neophaeosphaeria M.P.S. Câmara et al 2003 (C) $)^{\#}$

Nigrogranaceae Jaklitsch \& Voglmayr

Nigrograna Gruyter et al. 2012 (C)

Occultibambusaceae D.Q. Dai \& K.D. Hyde

Occultibambusa D.Q. Dai \& K.D. Hyde $2016(\mathbf{C})^{\# *}$

Seriascoma Phook. et al. $2016(\text { C })^{\# *}$

Versicolorisporium Sat. Hatak. et al. 2008

(C)

Ohleriaceae Jaklitsch \& Voglmayr

Ohleria Fuckel. 1868 (C) ${ }^{\#}$

Parabambusicolaceae Kaz. Tanaka \& K. Hiray.

Parabambusicola Kaz. Tanaka \& K. Hiray. $2015(\mathbf{H})^{\# *}$

Paradictyoarthriniaceae Doilom et al.

Paradictyoarthrinium Matsush. 1996 (H)*

Periconiaceae (Sacc.) Nann.
Periconia Tode 1791 (H)

Phaeosphaeriaceae M.E. Barr $(\mathbf{C})^{\# * *}$

Allophaeosphaeria Ariyaw. et al. 2015

Amarenographium O.E. Erikss. 1982 (C)

Ampelomyces Ces. ex Schltdl. 1852 (C)

Banksiophoma Crous 2017 (C)*

Camarosporioides W.J. Li \& K.D. Hyde

$2016(\mathbf{C})^{*}$

Chaetosphaeronema Moesz 1915 (C)

Mauginiella Cavara 1925 (H)

Melnikia Wijayaw. et al. 2016 (C)*

$(\mathbf{C})^{\# *}$

Muriphaeosphaeria Phukhams. et al. 2016

Neosetophoma Gruyter et al. 2010 (C)

Neosphaerellopsis Crous \& Trakun. 2014

(C)*

(C)*

Neostagonospora Quaedvl. et al. 2013

Neosulcatispora Crous \& M.J. Wingf. 2015 (C)*

Paraphoma Morgan-Jones \& J.F. White 1983 (C)

Parastagonospora Quaedvl. et al. 2013

(C)*

Phaeopoacea Thambug. et al. 2017 (C) ${ }^{\# *}$

Phaeosphaeria I. Miyake 1909 (C) ${ }^{\#}$

Phaeosphaeriopsis M.P.S. Câmara et al. 2003 (C) ${ }^{\#}$

Phaeostagonospora A.W. Ramaley 1997

Pleoseptum A.W. Ramaley \& M.E. Barr 1995 (C)

Poaceicola W.J. Li et al. 2015 (C) ${ }^{\# *}$

Sclerostagonospora Höhn. 1917 (C)

Scolicosporium Lib. ex Roum. 1880 (C)

Septoriella Oudem. 1889 (C)*

Setophoma Gruyter et al. 2010 (C)

Tiarospora Sacc. \& Marchal 1885 (C)

Vrystaatia Quaedvl. et al. 2013 (C)*

Wojnowiciella Crous et al. 2015 (C)*

Xenoseptoria Quaedvl. et al. 2013 (C)*

Pleomassariaceae M.E. Barr

Myxocyclus Riess 1852 asexual morph of

Splanchnonema sensu lato $(\mathbf{C})$

Prosthemium Kunze 1817 (C)\#

Pleomonodictydaceae Hern.-Restr. et al.

Pleomonodictys Hern.-Restr. et al. 2017 (H)* 
Pleosporaceae Nitschke

Alternaria Nees $1816(\mathbf{H})^{\#}$

Bipolaris Shoemaker 1959 (H)

Clathrospora Rabenh. $1857(\mathbf{H})^{\# *}$

Curvularia Boedijn 1933 (H)

Edenia M.C. González et al. 2007 (H)

(H) ${ }^{\#}$

Exserohilum K.J. Leonard \& Suggs 1974

Johnalcornia Y.P. Tan \& R.G. Shivas 2014 (H)*

Marielliottia Shoemaker 1999 (H)

Paradendryphiella Woudenberg \& Crous 2013 (H)*

Porocercospora Amaradasa et al. 2014

(H)*

Pithomyces Berk. \& Broome 1875 (H)

Pyrenophora Fr. 1849 (H)

Stemphylium Wallr. 1833 (H)

Tamaricicola Thambug. et al. $2016(\mathbf{C})^{\# *}$

Pseudocoleodictyosporaceae Doilom \& K.D. Hyde

Pseudocoleodictyospora Doilom \& K.D. Hyde 2016 (H)*

Pseudodidymellaceae A. Hashim. \& Kaz.

Tanaka

Petrakia Syd. \& P. Syd. 1913 (H) ${ }^{\# *}$

Pseudodidymella C.Z. Wei et al. 1997 (H)

Xenostigmina Crous 1998 (H)*

Roussoellaceae J.K. Liu et al.

Cytoplea Bizz. \& Sacc. 1885 (C)

Immotthia M.E. Barr 1987 (C) ${ }^{\# *}$

Roussoella Sacc. $1888(\text { C) })^{\# *}$ $(\mathrm{C})^{\# *}$

Roussoellopsis I. Hino \& Katum. 1965

\section{Sporormiaceae Munk}

Forliomyces Phukhams. et al. 2016 (C)*

Westerdykella Stolk 1955 (C) ${ }^{\#}$

Sparticola Phukhams. et al. 2016 (H) ${ }^{\# *}$

Sulcatisporaceae Kaz. Tanaka \& K. Hiray.

Magnicamarosporium Kaz. Tanaka \& K.

Hiray. 2015 (C)*

Neobambusicola Crous \& M.J. Wingf. $2014(\mathbf{C})^{*}$

Sulcatispora Kaz. Tanaka \& K. Hiray. $2015(\mathbf{C})^{\#_{*}}$

Teichosporaceae M.E. Barr
Magnibotryascoma Thambug. \& K.D. Hyde 2015 (C)*

Paulkirkia Wijayaw. et al. 2016 (C)*

Teichospora Fuckel 1870 (C)

Tetraplosphaeriaceae Kaz. Tanaka \& K. Hiray Polyplosphaeria Kaz. Tanaka \& K. Hiray. $2009(\mathbf{H})^{\#}$

Pseudotetraploa Kaz. et al. 2009 (H)

Quadricrura Kaz. Tanaka et al. 2009 (H)

Shrungabeeja V.G. Rao \& K.A. Reddy $1981(\mathbf{H})^{*}$

Tetraploa Berk. \& Broome 1850 (H)

Torulaceae Corda*

Dendryphion Wallr. 1833 (H)*

Neotorula Ariyaw. et al. 2016 (H)*

Sporidesmioides J.F. Li et al. 2016 (H)*

Torula Pers. 1795 (H)

Trematosphaeriaceae K.D. Hyde et al.

Medicopsis Gruyter et al. 2012 (C)*

Trematosphaeria Fuckel 1870 (C) ${ }^{\# *}$

Zopfiaceae G. Arnaud ex D. Hawksw. 1992

Zopfia Rabenh. 1874 (H) ${ }^{\#}$

Pleosporales, genera incertae sedis

Aegeanispora E.B.G. Jones \& Abdel-

Wahab 2017 (C)*

Antealophiotrema A. Hashim. \& Kaz.

Tanaka $2017(\mathbf{H})^{\# *}$

Bactrodesmium Cooke 1883 (H)*

Bahusandhika Subram. 1956 (H)

Berkleasmium Zobel 1854 (H)

Briansuttonia R.F. Castañeda et al. 2004

(H)

Camarographium Bubák 1916 (C)

Cerebella Ces. 1851 (H)

Chaetodiplodia P. Karst. 1884 (C)

Chaetophoma Cooke 1878 (C)

Cheiromoniliophora Tzean \& J.L. Chen 1990 (H)

Cyclothyrium Petr. 1923 (C)

Dokmaia I. Promputtha 2003 (H)*

Foliophoma Crous 2017 (C)*

Fusculina Crous \& Summerell 2006 (C)

Gordonomyces Crous \& Marinc. 2011 (C)

(H)*

Megacapitula J.L. Chen \& Tzean 1993

Mycocentrospora Deighton 1972 (H)

Noosia Crous et al. 2011 (H) 
Ochrocladosporium Crous \& U. Braun 2007 (H)

Paraepicoccum Matsush. 1993 (H)*

Parameliola Hongsanan et al. 2016 (H)*

Perthomyces Crous 2017 (C)*

Phialophorophoma Linder 1944 (C)

Pleiochaeta (Sacc.) S. Hughes 1951 (H)

Polyschema H.P. Upadhyay 1966 (H)

Pseudochaetosphaeronema Punith. 1979

(C)

Pseudopassalora Crous 2011 (H)

Pseudoxylomyces Kaz. Tanaka \& K. Hiray. 2015 (H)*

Repetophragma Subram. 1992 (H)

Rutola J.L. Crane \& Schokn. 1978 (H)*

Scolecobasidium E.V. Abbott 1927 (H)

Scleroramularia Batzer \& Crous 2011 (H)

Setophaeosphaeria Crous \& Y. Zhang ter $2014(\mathbf{C})^{\# *}$

Sirodesmium De Not. 1849 (C)

Spiroplana Voglmayr et al. 2011 (H)

Thyrostroma Höhn. 1911 (H)

Pleosporomycetidae, genera incertae sedis

Acrogenospora M.B. Ellis 1971 (H)

Dothideomycetes orders incertae sedis

Acrospermales Minter et al.

Acrospermaceae Fuckel

Acrospermum Tode 1783 with dactylarialike asexual morph (H) ${ }^{\#}$

Gonatophragmium Deighton 1969

Acrospermales genera incertae sedis

Paramycoleptodiscus Crous \& M.J. Wingf. $2016(\mathbf{C})^{*}$

Asterinales M.E. Barr ex D. Hawksw. \& O.E.

Erikss.

Asterinaceae Hansf.

Blastacervulus H.J. Swart 1988 (C)

Discopycnothyrium Hongsanan \& K.D.

Hyde 2017 (C)*

Eupelte Syd. $1924(\mathbf{H})^{\# *}$

Pirozynskiella S. Hughes 2007 (H)

Prillieuxina G. Arnaud 1918 (C) ${ }^{\#_{*}}$

Uleothyrium Petr. 1929 (C) ${ }^{\#_{*}}$

Botryosphaeriales C.L. Schoch et al.

Aplosporellaceae Slippers et al.

Aplosporella Speg. 1880 (C)
Botryosphaeriaceae Theiss. \& H. Syd.

Alanphillipsia Crous \& M.J. Wingf. 2013

(C)*

Barriopsis A.J.L. Phillips et al. 2008 (C)

Botryosphaeria Ces. \& De Not. 1863 (C) ${ }^{\# *}$

Cophinforma Doilom et al. 2012 (C)

Diplodia Fr. 1834 (C)

Dothiorella Sacc. 1880 (C)

Endomelanconiopsis E.I. Rojas \& Samuels 2008 (C)

Eutiarosporella Crous 2015 (C) \#* $^{*}$

Lasiodiplodia Ellis \& Everh. 1896 (C)

Macrophomina Petr. 1923 (C)

Marasasiomyces Crous 2015 (C)*

Microdiplodia Allesch. 1901 (C)

Mucoharknessia Crous et al. 2015 (C)*

Neodeightonia C. Booth 1970 (C) ${ }^{\# *}$

Neofusicoccum Crous et al. 2006 (C)

Phaeobotryon Theiss. \& Syd. 1915 (C)

Sakireeta Subram. \& K. Ramakr. 1957

(C)*

Sardiniella Linaldeddu et al. 2016 (C)*

Spencermartinsia A.J.L. Phillips et al. 2008 (C) $)^{\#}$

Sphaeropsis Sacc. $1880(\mathbf{C})^{\#}$

Tiarosporella Höhn. 1919 (C)

Planistromellaceae M.E. Barr

Comminutispora A.W. Ramaley 1996 (H)

Kellermania Ellis \& Everh. 1885 (C)

Microcyclus Sacc. et al. 1904 with aposphaeria-like asexual morph (C) ${ }^{\#}$

Umthunziomyces Crous \& M.J. Wingf. $2016(\mathbf{C})^{*}$

Saccharataceae Slippers et al.

Neoseptorioides Crous et al. 2015 (C)*

Saccharata Denman \& Crous 2004 (C)

Septorioideaceae Wyka \& Broders

Septorioides Quaedvl. et al. 2013 (C)*

Collemopsidiales Pérez-Ortega

Xanthopyreniaceae Zahlbr.

Collemopsidium Nyl. 1881 (C)

Jahnulales Pang et al.

Aliquandostipitaceae Inderbitzin

Brachiosphaera Nawawi 1976 (H)

Xylomyces Goos et al. 1977 (H)

Kirschsteiniotheliales Hern.-Restr. et al. 
Kirschsteiniotheliaceae S. Boonmee \& K.D. Hyde* $(\mathbf{H})^{\# *}$

Kirschsteiniothelia D. Hawksw. 1985

Solicorynespora R.F. Castañeda \& W.B. Kendr. 1990 (H)

Kirschsteiniotheliales genera incertae sedis Brachysporiella Bat. 1952 (H)*

Lichenoconiales Diederich et al.

Lichenoconiaceae Diederich \& Lawrey Lichenoconium Petr. \& Syd. 1927 (C)

Microthyriales G. Arnaud

Micropeltidaceae Clem. \& Shear

Heliocephala V.G. Rao et al. 1984 (H)

Stomiopeltis Theiss. 1914 (as

Akaropeltella M.L. Farr 1972) with sporidesmium-like asexual morph $(\mathbf{H})^{\#}$

Microthyriaceae Sacc.

Asterinella Theiss. 1912 with asteromellalike asexual morph (C) ${ }^{\#}$

Hansfordiella S. Hughes 1951 (H)

Leptothyrium Kunze 1823 (C)

Microthyriales genera incertae sedis

Neoscolecobasidium Crous 2016 (H)*

Stigmatodiscales Voglmayr \& Jaklitsch

Stigmatodiscaceae Voglmayr \& Jaklitsch

Asterodiscus Voglmayr et al. 2016 (C) ${ }^{\# *}$ $(\mathbf{C})^{\# *}$

Stigmatodiscus Voglmayr \& Jaklitsch 2016

Valsariales Jaklitsch et al.

Valsariaceae Jaklitsch et al.

Valsaria Ces. \& De Not. 1863 (C) ${ }^{\# *}$

Venturiales Yin. Zhang et al.

Venturiaceae E. Müll. \& Arx ex M.E. Barr

Apiosporina Höhn. 1910 with

cladosporium-like asexual morph $\mathbf{( H )}{ }^{\#}$

Dictyodochium Sivan. 1984 (H)

Fusicladosporium Partr. \& Morgan-Jones

2003 (H)

Magnohelicospora R.F. Castañeda et al. 2013 (H)*

Protoventuria Berl. \& Sacc. 1887 (H) ${ }^{\#}$

Spilocaea Fr. 1819 (C)
Sympoventuriaceae Yin. Zhang et al. Acroconidiellina (Berk. \& Broome) M.B. Ellis 1971 (H) ${ }^{\#}$

Fusicladium Bonord. 1851 (H)

Mycosisymbrium Carris 1994 (H)

Ochroconis de Hoog \& Arx 1974 (H) ${ }^{\# *}$

Sympoventuria Crous \& Seifert with sympodiella-like asexual morph of $\mathbf{( H )}{ }^{\#}$

Veronaeopsis Arzanlou \& Crous 2007 (H)

Verruconis Samerp. et al. 2014 (H)*

Venturiales genera incertae sedis

Cylindrosympodioides Crous \& M.J. Wingf. 2016 (H) *

Cylindrosympodium W.B. Kendr. \& R.F. Castañeda 1990 (H)

Superstratomycetales van Nieuwenhuijzen et al.

Superstratomycetaceae van Nieuwenhuijzen et al.

Superstratomyces van Nieuwenhuijzen et al. 2017 (C)*

Tubeufiales Boonmee \& K.D. Hyde*

Tubeufiaceae M.E. Barr

Acanthohelicospora Boonmee \& K.D. Hyde $2014(\mathbf{H})^{\# *}$

Aquaphila Goh et al. 1998 (H)

Chlamydotubeufia Boonmee \& K.D. Hyde $2011(\mathbf{H})^{\# *}$

$(\mathbf{H})^{\# *}$

Dictyospora Brahamanage et al. 2017

Helicangiospora Boonmee et al. $2014(\mathbf{H})^{\#}$

Helicoma Corda $1837(\mathbf{H})$

Helicomyces Link 1809 (H)

Helicosporium Nees $1816(\mathbf{H})^{\#_{*}}$

Manoharachariella Bagyan. et al. 2009

(H)

Moorella P. Rag. Rao \& D. Rao 1964 (H)

Muripulchra Z.L. Luo et al. 2017 (H)*

Neoacanthostigma Boonmee et al. 2014 (H)*

Neohelicomyces Z.L. Luo et al. 2017 (H)*

Paranectriella (P. Henn. \& Sacc.) Höhn. $1910(\mathbf{H})^{\#}$

Tamhinispora K.C. Rajeshk. \& Rahul Sharma 2013 (H)*

Tubeufia Penz. \& Sacc. $1898(\mathbf{H})^{\#_{*}}$

Wiesneriomycetaceae Suetrong et al.* 
Parawiesneriomyces Crous \& M.J. Wingf. 2016 (H)*

Pseudogliophragma Phadke \& V.G. Rao 1980 (H)

Wiesneriomyces Koord. 1907 (H)*

Zeloasperisporiales Hongsanan \& K.D. Hyde

Zeloasperisporiaceae Crous (H)*

Zeloasperisporium R.F. Castañeda 1996

Dothideomycetes, families incertae sedis

Ascoporiaceae Kutorga \& D. Hawksw.

Pseudosolidum Lloyd 1923 with

plectophomella-like asexual morph $(\mathbf{H})^{\#}$

Englerulaceae Henn.

Capnodiastrum Speg. 1886 (C)

Digitosarcinella S. Hughes 1984 (H)

Sarcinella Sacc. 1880 (H)

Eremomycetaceae Malloch \& Cain

Arthrographis G. Cochet ex Sigler \& J.W.

Carmich. 1976 (H)

Homortomycetaceae Thambug. et al.

Homortomyces Crous \& M.J. Wingf. 2012

(C)

Leptopeltidaceae Höhn. ex Trotter

Leptopeltis Höhn. 1917 with leptothyriumlike asexual morph (C) ${ }^{\#}$

Paranectriellaceae S. Boonmee \& K.D. Hyde Araneomyces Höhn. $1909(\mathbf{H})^{\#}$

Puttemansia Henn. 1902 (H)

Perisporiopsidaceae E. Müll. \& Arx ex R. Kirschner \& T.A. Hofm.

Neoparodia Petr. \& Cif. 1932 with sarcinella-like asexual morph $\mathbf{( H )}{ }^{\#}$

Schizothyriaceae Höhn. ex Trotter et al. Zygophiala E.W. Mason 1945 (H)

\section{Vizellaceae H.J. Swart}

Blasdalea Sacc. \& P. Syd. 1902 (C)

Licopolia Sacc. et al. $1900(\mathbf{C})^{\# *}$

Dothideomycetes, genera incertae sedis Acanthorus Bat. \& Cavalc. 1967 (C) Ampullifera Deighton 1960 (H)
Anguillosporella U. Braun 1995 (H)

Arxiella Papendorf 1967 (H)

Asterodothis Theiss. 1912 (C)

Asteromella Pass. \& Thüm. 1880 (C)

Bahusakala Subram. 1958 (H)

Botryohypoxylon Samuels \& J.D. Rogers $1986(\mathbf{C})^{\#}$

Brachyconidiella R.F. Castañeda \& W.B. Kendr. 1990 (H)

Brooksia Hansf. 1956 (H)

Ceratophoma Höhn. 1917 (C)

Chaetosticta Petr. \& Syd. 1925 (C)

Chionomyces Deighton \& Piroz. 1972 (H)

Chuppia Deighton 1965 (H)

Cladoriella Crous 2006 (H)

Clavariopsis De Wild. 1895 (H)

Cryomyces Selbmann et al. 2005 (H)*

Cyclopeltella Petr. 1953 (C) ${ }^{\#}$

Dactuliophora C.L. Leakey 1964 (H)

Dilophospora Desm. 1840 (C)

Dothichiza Lib. ex Roum. 1880 (C)

Endosporium Tsuneda 2008 (H)

Eriomycopsis Speg. 1910 (H)

Eriothyrium Speg. 1888 (C)

Excipulariopsis P.M. Kirk \& Spooner 1982 (H) ${ }^{\#}$

Funbolia Crous \& Seifert 2011 (H)

Fusicladiella Höhn. 1919 (H)

Gibbago E.G. Simmons 1986 (H)

Hansfordiellopsis Deighton 1960 (H)

Heptaster Cif. et al. 1956 (H)

Heteroconium Petr. 1949 (H)

Hobsoniopsis D. Hawksw. 2001 (H)

Hormiokrypsis Bat. \& Nascim. 1957 (H)

Kabatia Bubák 1904 (C)

Krishnamyces Hosag. 2003 (C)

Lignosphaeria Boonmee et al. $2015(\mathbf{C})^{\# *}$

Manginula G. Arnaud 1918 (C)

Monodictys S. Hughes 1958 (H)

Neodactylaria Guevara-Suarez et al. 2017

(H)*

Neoovularia U. Braun 1992 (H)

Neoramularia U. Braun 1991 (H)

Neosporidesmium Mercado \& J. Mena 1988 (H)

Neottiosporina Subram. 1961 (C)

Oncopodiella G. Arnaud ex Rifai 1965 (H)

Ophiotrichum Kunze 1849 (H)

Pazschkeella Syd. \& P. Syd. 1901 (C)

Peltaster Syd. \& P. Syd. 1917 (C)

Peltasterella Bat. \& H. Maia 1959 (C)

Pendulispora M.B. Ellis 1961 (H) 
Perusta Egidi \& Stielow 2014 (H)*

Phaeoramularia Munt.-Cvetk. 1960 (H)

Phaeosclera Sigler et al. 1981 (H)

Phanerococculus Cif. 1954 (C)

Pirozynskia Subram. 1972 (H)

Placosphaeria (De Not.) Sacc. 1880 (C)

Plectopycnis Bat. \& A.F. Vital 1959 (C)

Plenotrichaius Bat. \& Valle 1961 (C)

Podoplaconema Petr. 1921 (C)

Prathoda Subram. 1956 (H)

Proliferosphaera T.P. Devi 2012 (C)*

Pseudodidymella C.Z. Wei et al. 1997 (H)

Pseudorobillarda M. Morelet 1968 (C)

Pseudovirgaria H.D. Shin et al. 2007 (H)

Quasiphloeospora B. Sutton et al. 1996 (H)

Radulidium Arzanlou et al. 2007 (H)

Ramulariopsis Speg. 1910 (H)

Rhabdospora (Durieu \& Mont. ex Sacc.)

Sacc. 1884 (C)

Rupestriomyces Lei Su et al. 2015 (H)*

Sarcinomyces Lindner 1898 (H)

Saxomyces L. Selbmann \& D. Isola 2014

(H)*

Scleroconidioma Tsuneda et al. 2000 (H)

Scolecoxyphium Cif. \& Bat. 1956 (C)

Septoidium G. Arnaud 1921 (H)

Shearia Petr. 1924 (C)

Shivamyces Hosag. 2004 (H)

Sirosporium Bubák \& Serebrian. 1912 (H)

Soloacrosporiella Crous \& M.J. Wingf. 2015 (H)*

Speiropsis Tubaki 1958 (H)

Spermatoloncha Speg. 1908 (H)

Spissiomyces Lei Su et al. 2015 (H)*

Sporidesmajora Batzer \& Crous 2010 (H)

Tetracrium Henn. 1902 (H)

Torulopsiella Bender 1932 (H)

Tretospora M.B. Ellis 1976 (H)

Troposporella P. Karst. 1892 (H)

Verrucisporota D.E. Shaw \& Alcorn 1993

(H)

(C)

Vouauxiomyces Dyko \& D. Hawks. 1979

Xenophacidiella Crous 2011 (H)

Xenosporium Penz. \& Sacc. 1901 (H)

Yoshinagaia Henn. 1904 (C)

Class Eurotiomycetes Tehler ex O.E. Eriksson \& K. Winka

Subclass Chaetothyriomycetidae Doweld

Chaetothyriales M.E. Barr

Chaetothyriaceae Hansf. ex M.E. Barr
Aphanophora Réblová \& Unter. 2013 (H)*

Camptophora Réblová \& Unter. 2013 (H)*

Ceramothyrium Bat. \& H. Maia 1956 (H)

Cyphellophoriella Crous \& A. J. Smith 2015 (H)*

Vonarxia Bat. 1960 (H)

Coccodiniaceae Höhn. ex O.E. Erikss.

Microxiphium (Harv. ex Berk. \& Desm.) Thüm. 1879 (H)

Cyphellophoraceae Réblová \& Unter.

Cyphellophora G.A. de Vries 1962 (H)*

Herpotrichiellaceae Munk

Cladophialophora Borelli 1980 (H)

Exophiala J.W. Carmich. 1966 (H)

Fonsecaea Negroni 1936 (H)

Melanoctona Qing Tian et al. $2016(\mathbf{H})^{\# *}$

Metulocladosporiella Crous et al. 2006 (H)

Minimelanolocus R.F. Castañeda \&

Heredia 2001 (H)*

Nadsoniella Issatsch. 1914 (H)

Phialophora Medlar 1915 (H)

Rhinocladiella Nannf. 1934 (H)

Sorocybe Fr. 1849 (H)

Thysanorea Arzanlou et al. 2007 (H)

Veronaea Cif. \& Montemart. 1957 (H)

Lyrommataceae Lücking

Lyromma Bat. \& H. Maia 1965 (C)

Strelitzianaceae Crous \& M.J. Wingf.

Neophaeococcomyces Crous \& M.J.

Wingf. 2015 (H)*

Strelitziana Arzanlou \& Crous 2006 (H)*

Trichomeriaceae Chomnunti \& K.D. Hyde

Arthrocladium Papendorf 1969 (H)*

Bradymyces Hubka et al. 2014 (Y)*

Brycekendrickomyces Crous \& M.J.

Wingf. 2009 (H)

Knufia L.J. Hutchison \& Unter. 1996 (H)

Lithohypha Selbmann \& Isola 2017 (H)*

Chaetothyriales, genera incertae sedis Atrokylindriopsis Y.R. Ma \& X.G. Zhang 2015 (H)*

(C)

Lichenodiplis Dyko \& D. Hawksw. 1979

Melnikomyces Crous \& U. Braun 2014

(H)* 
Neostrelitziana Crous \& M.J. Wingf. 2015 (H)*

Paracladophialophora Crous 2016 (H)* $1979(\mathbf{H})$

Uncispora R.C. Sinclair \& Morgan-Jones

Phaeomoniellales K.H. Chen et al.

Phaeomoniellaceae P.M. Kirk

Aequabiliella Crous 2015 (C)*

Celerioriella Crous 2015 (H)*

Minutiella Crous 2015 (H)*

Neophaeomoniella Rooney-Latham \& Crous 2015 (C)*

Paraphaeomoniella Crous 2015 (C)*

Phaeomoniella Crous \& W. Gams 2000

(H)

(C)*

Pseudophaeomoniella Nigro et al. 2015

Xenocylindrosporium Crous \& Verkley 2009 (C)

Chaetothyriomycetidae, genera incertae sedis

Dolabra C. Booth \& W.P. Ting 1964 with rhabdospora-like asexual morph $(\mathbf{C})^{\#}$

Subclass Eurotiomycetidae Geiser \& Lutzoni Arachnomycetales Gibas et al. Arachnomycetaceae Gibas et al.

Onychocola Sigler 1990 (H)

Eurotiales G.W. Martin ex Benny \& Kimbr. Aspergillaceae Link

Aspergillus P. Micheli ex Link 1809 (H)

Hamigera Stolk \& Samson 1971 (H)

Penicillium Link 1809 (H) ${ }^{\#}$

Phialomyces P.C. Misra \& P.H.B. Talbot 1964 (H)

Monascaceae J. Schröt.

Fraseriella Cif. \& A.M. Corte 1957 (H)

Monascus Tiegh. 1884 (H)

Thermoascaceae Apinis

Paecilomyces Bainier 1907 (H) ${ }^{\# *}$

Trichocomaceae E. Fisch.

Sagenomella W. Gams 1978 (H)

Talaromyces C.R. Benj. 1955 (H)

Thermomyces Tsikl. 1899 (H)

Onygenales Cif. ex Benny \& Kimbr.

Ajellomycetaceae Untereiner et al.
(H)

Blastomyces Gilchrist \& W.R. Stokes 1898

Histoplasma Darling 1906 (H)

Paracoccidioides F.P. Almeida 1930 (H)

Arthrodermataceae Currah

Microsporum Gruby 1843 (H)

Trichophyton Malmsten 1848 (H)

Ascosphaeraceae L.S. Olive \& Spiltoir

Ascosphaera L.S. Olive \& Spiltoir 1955 with chrysosporium-like asexual morph (H) ${ }^{\#}$

Bettsia Skou 1972 with chrysosporium-like asexual morph $\mathbf{( H )}{ }^{\#}$

Gymnoascaceae Baran.

Oncocladium Wallr. 1833 (H)

Orromyces B. Sur \& G.R. Ghosh 1987

with chrysosporium-like asexual morph (H) ${ }^{\#}$

Nannizziopsidaceae Guarro et al.

Nannizziopsis Currah 1985 (H)

Onygenaceae Berk.

Chrysosporium Corda 1833 (H)

Coccidioides G.W. Stiles 1896 (H)

Malbranchea Sacc. 1882 (H)

Myriodontium Samson \& Polon. 1978 (H)

Ophidiomyces Sigler et al. 2013 (H)*

Paranannizziopsis Sigler et al. 2013 (H)*

Sporendonema Desm. 1827 (H)

Spiromastigaceae Hirooka et al.

Sigleria Hirooka et al. 2016 (H)*

Spiromastix Kuehn \& G.F. Orr 1962 (H)

Onygenales, genera incertae sedis

Arthropsis Sigler et al. 1982 (H)

Lacazia Taborda et al. 1999 (H)

Sphaerosporium Schwein. 1832 (H)

Subclass Mycocaliciomycetidae Tibell

Mycocaliciales Tibell \& Wedin

Mycocaliciaceae A.F.W. Schmid

Chaenothecopsis Vain. 1927 (H)

Mycocalicium Vain. ex Reinke 1890 (H) ${ }^{\#_{*}}$

Eurotiomycetes, genera incertae sedis

Cirrosporium S. Hughes 1980 (C)

Neocladophialophora Crous \& R.K.

Schumach. 2014 (H)* 
Class Laboulbeniomycetes Engler

Laboulbeniales genera incertae sedis

Coreomycetopsis Thaxt. 1920 (H)

Muiaria Thaxt. 1914 (H)

Pyxidiophorales P.F. Cannon

Pyxidiophoraceae Arnold

Gliocephalis Matr. 1899 (H)

Pleurocatena G. Arnaud ex Aramb. et al. 2007 (H)

Class Lecanoromycetes O.E. Erikss. \& Winka

Subclass Ostropomycetidae Reeb et al.

Ostropales Nannf.

Stictidaceae Fr.

Acarosporina Sherwood 1977 with phacidiella-like asexual morph $(\mathbf{C}){ }^{\#}$

Cyanodermella O.E. Erikss. 1981 (C)

Glomerobolus Kohlm. \& Volkm.-Kohlm. 1996 (H)

Stictis Pers. 1800 (C)

Stictophacidium Rehm 1888 with coleophoma-like asexual morph (C) ${ }^{\#}$

Subclass Lecanoromycetidae P.M. Kirk et al.

Lecanorales Nannf.

Stereocaulaceae Chevall.

Lepraria Ach. 1803 (C)

Lecanorales genera incertae sedis

Lichenosticta Zopf 1898 (C)

Leprocaulales Lendemer \& B.P. Hodk.

Leprocaulaceae Lendemer \& B.P. Hodk.

Leprocaulon Nyl. 1878 (C)

Ostropales Nannf.

Graphidaceae Dumort.

Rubikia H.C. Evans \& Minter 1985 (C)

Ostropales genera incertae sedis

Mulderomyces Crous et al. 2016 (C)*

Peltigerales Walt. Watson

Collemataceae Zenker

Paracollema Otálora \& Wedin $2014(\mathbf{C})^{\# *}$

Class Leotiomycetes O.E. Erikss. \& Winka

Erysiphales Gwynne-Vaughan

Erysiphaceae Tul. \& C. Tul.

Blumeria Golovin ex Speer 1975 (H)

Erysiphe R. Hedw. ex DC. 1805 (H)
Leveillula G. Arnaud 1921 (H)

Phyllactinia Lév. 1851 (H)

Helotiales Nannf.

Ascodichaenaceae D. Hawksw. \& Sherwood Ascodichaena Butin 1977 (C)

Bloxamiaceae Locq.

Bloxamia Berk. \& Broome 1854 (H)

Calloriaceae Marchand

Calloria Fr. $1836(\mathbf{H})^{\# *}$

Cenangiaceae Rehm

Crumenulopsis J.W. Groves 1969 (C) ${ }^{\# *}$

Rhabdocline Syd. 1922 (H)

Chaetomellaceae Baral et al.

Chaetomella Fuckel 1870 (C) \#

Pilidium Kunze 1823 (C) ${ }^{\#}$

Sphaerographium Sacc. 1884 (C) ${ }^{\#}$

(H)

Synchaetomella Decock \& Seifert 2005

Chlorociboriaceae Baral \& P.R. Johnst.

Chlorociboria Seaver ex Ramamurthi et al. $1958(\mathbf{C})^{\#}$

Cordieritidaceae Sacc.

Diplolaeviopsis Giralt \& D. Hawksw. 1991

(C)*

Unguiculariopsis Rehm 1909 (C)

Dermateaceae Fr.

Coleophoma Höhn. 1907 (C)

Corniculariella P. Karst. 1884 (C)

Dermea Fr. 1825 (C) ${ }^{\# *}$

Neofabraea H.S. Jacks. 1913 (C)

Parafabraea Chen Chen et al. 2015 (C) ${ }^{\# *}$

Pezicula Tul. \& C. Tul. 1865 (C)

Phialocephala W.B. Kendr. 1961 (H)

Phlyctema Desm. 1847 (C) $(\mathbf{C})^{\# *}$

Verkleyomyces Y. Marín \& Crous 2016

Xenochalara M.J. Wingf. \& Crous 2000

(H)

Drepanopezizaceae Bat. \& H. Maia

Blumeriella Arx $1961(\mathbf{C})^{\# *}$

Diplocarpon F.A. Wolf 1912 (C) or $(\mathbf{H})^{\# *}$

Leptotrochila P. Karst. 1871 (C) ${ }^{\# *}$ 
Pseudopezicula Korf 1975 with phialophora-like asexual morph (H) ${ }^{\#}$

Gelatinodiscaceae S.E.Carp

Ascocoryne J.W. Groves \& D.E. Wilson $1967(\mathbf{H})^{\# *}$

Neobulgaria Petr. 1921 with myrioconium-like or phialophora-like asexual morph (H) ${ }^{\#}$

Xerombrophila Baral $2013(\mathbf{H})^{\#_{*}}$

\section{Godroniaceae Baral}

Ascocalyx Naumov 1926 (C)

Godronia Moug. \& Lév. 1846 (C)

Gremmeniella M. Morelet 1969 (C)

Gloeotinia M. Wilson et al. 1954 (H)

\section{Helotiaceae Rehm}

Articulospora Ingold 1942 (H)

Ascoconidium Seaver $1942(\mathbf{H})^{\# *}$

Dimorphospora Tubaki 1958 (H)

Filosporella Nawawi 1976 (H)*

Fontanospora Dyko 1978 (H)

Geniculospora Sv. Nilsson ex Marvanová \& Sv. Nilsson $1971(\mathbf{H})$

Glarea Bills \& Paláez 1999 (H)

Helicodendron Peyronel 1918 (H)

Hymenoscyphus Gray 1821 (H) ${ }^{\#}$

Mycofalcella Marvanová et al. 1993 (H)*

Neocrinula Crous 2016 (H)*

Scytalidium Pesante 1957 (H)

Symphyosirinia E.A. Ellis 1956 (H)

Tricladium Ingold 1942 (H)

\section{Heterosphaeriaceae Rehm}

Heterosphaeria Grev. 1824 (C)

Hyaloscyphaceae Nannf.

(H)

Arbusculina Marvanová \& Descals 1987

Cistella Quél. $1886(\mathbf{H})^{\# *}$

Cladochasiella Marvanová 1997 (H)

Clathrosphaerina Beverw. 1951 (H)

Crucellisporiopsis Nag Raj 1983 (C)*

Dematioscypha Svrček 1977 (H)

Hyalopeziza Fuckel 1870 (H) ${ }^{\# *}$

Hyaloscypha Boud. $1885(\mathbf{H})^{\# *}$

Hyphodiscus Kirschst. 1906 (H)

Meliniomyces Hambl. \& Sigler 2005 (H)

Mycoarthris Marvanová \& P.J. Fisher $2002(\mathbf{H})$
(H)

Leptodontidiaceae Hern.-Restr. et al. Leptodontidium de Hoog. 1979 (H)

Mollisiaceae Rehm

Cystodendron Bubák 1914 (H)

Fuscosclera Hern.-Restr. et al. 2017 (H)*

Trimmatostroma Corda 1837 (H)*

Variocladium Descals \& Marvanová (H)

Pezizellaceae Velen.

Calycellina Höhn 1918 (H)

Chalara (Corda) Rabenh. 1844 (H)

Ciliolarina Svrcek 1977 (H) ${ }^{*} *$

Scleropezicula Verkley $1999(\mathbf{C})^{\# *}$

Ploettnerulaceae Kirschst.

Cadophora Lagerb. \& Melin (H)

Mycochaetophora Hara \& Ogawa (H)

Oculimacula Crous \& W. Gams 2003 (H)

Pyrenopeziza Fuckel $1870(\mathbf{C})^{\# *}$

Rhynchosporium Heinsen ex A.B. Frank 1897 (H)

Rutstroemiaceae Holst-Jensen et al.

Rutstroemia P. Karst. 1871 with phialophora-like asexual morph (H) ${ }^{\#}$

Sclerotiniaceae Whetzel ex Whetzel

Amphobotrys Hennebert 1973 (H)

Botrytis P. Micheli ex Pers. 1794 (H) ${ }^{\#}$

Cristulariella Höhn. 1916 (H)

Grovesinia M.N. Cline et al. 1983 (H)

Haradamyces Masuya et al. 2009 (H)

Monilinia Honey 1928 (H) ${ }^{\#}$

Mycopappus Redhead \& G.P. White 1985

(H)

Myriosclerotinia N.F. Buchw. 1947 (H) ${ }^{\#}$

Ovulinia F.A. Weiss 1940 (H) ${ }^{\#}$

(C) ${ }^{\#}$

Pycnopeziza W.L. White \& Whetzel 1938

Sclerotium Tode 1790 (H)

Seaverinia Whetzel 1945 (H)

Septotinia Whetzel ex J.W. Groves \& M.E. Elliott $1961(\mathbf{H})^{\#}$

Streptotinia Whetzel 1945 (H)

Valdensia Peyronel 1923 (H)

Vibrisseaceae Korf

Acephala Grünig \& T.N. Sieber 2005 (H) 
Chlorovibrissea L.M. Kohn 1989 with phialophora-like asexual morph (H) ${ }^{\#}$

Vibrissea Fr. 1822 (H) ${ }^{\#}$

Helotiales, genera incertae sedis

Acidea Hujslová \& M. Kolařík 2014 (H)*

Acidomelania E. Walsh \& N. Zhang 2014 (H)*

Apiculospora Wijayaw. et al. 2016 (C)*

Barrenia E. Walsh \& N. Zhang 2015 (H)*

Bioscypha Syd. 1927 with rhinocladiellalike asexual morph (H) ${ }^{\#}$

(H)

Brachyalara Réblová \& W. Gams 2011

Brefeldochium Verkley 2005 (H)

Cheirospora Moug. \& Fr. 1825 (C)

Clathrosporium Nawawi \& Kuthub. 1987

(H)

Collembolispora Marvanová \& Pascoal $2003(\mathbf{H})^{*}$

Crucellisporium M.L. Farr 1968 (C)

Dactylaria Sacc. 1880 (H)

Fulvoflamma Crous 2006 (H)

$(\mathbf{C})^{\# *}$

Godroniopsis Diehl \& E.K. Cash 1929

Gyoerffyella Kol 1928 (H)

Humicolopsis Cabral \& S. Marchand 1976

(H)

Hyalodendriella Crous 2007 (H)

Infundichalara Réblová \& W. Gams 2011 (H)

Lareunionomyces Crous \& M.J. Wingf. $2016(\mathbf{H})^{*}$

Lemonniera De Wild. 1894 (H)

Leptodontidium de Hoog 1979 (H)

Libartania Nag Raj 1979 (C)

Margaritispora Ingold 1942 (H)

Mastigosporium Riess 1852 (H)*

Monochaetiellopsis B. Sutton \& DiCosmo 1977 (C) ${ }^{\#}$

Phacidiella P. Karst. 1884 (C)

Rhexocercosporidium U. Braun 1994 (H)

Rhizocladosporium Crous \& U. Braun 2007 (H)

Rhizothyrium Naumov 1915 (C)

)

(H)*

Sabahriopsis Crous \& M.J. Wingf. 2015

Soosiella Hujslová \& M. Kolařík 2014 (H)*

Spirosphaera Beverw. 1953 (H)

Stamnaria Fuckel 1870 (C)
Strossmayeria Schulzer 1881 (H)

Tetracladium De Wild. 1893 (H)

Thedgonia B. Sutton 1973 (H)

Triposporium Corda 1837 (H)

Varicosporium W. Kegel 1906 (H)

Xenopolyscytalum Crous 2010 (H)

Lauriomycetales Hern.-Restr. et al.

Lauriomycetaceae Hern.-Restr. et al. Lauriomyces R.F. Castañeda 1990 (H)

Leotiales Carpenter

Leotiaceae Corda

Alatospora Ingold 1942 (H)

Flagellospora Ingold 1942 (H)

Halenospora E.B.G. Jones 2009 (H)

Miniancora Marvanová \& Bärl. 1989 (H)

Phacidiales C.E. Bessey

Helicogoniaceae Baral

Eleutheromycella Höhn. 1908 (C)

Eleutheromyces Fuckel 1870 (C)

$(\mathbf{H})^{\# *}$

Gelatinipulvinella Hosoya \& Y. Otani 1995

Phacidiaceae Fr.

Allantophomopsiella Crous 2014 (C)*

Allantophomopsis Petr. 1925 (C)

Darkera H.S. Whitney et al. (C)

Phacidiopycnis Potebnia 1912 (C)

Phacidium Fr. 1815 (C)

Tympanidaceae Baral \& Quijada

Claussenomyces Kirschst. $1923(\mathbf{H})^{\# *}$

Collophorina Damm \& Crous 2017 (C)

Holwaya Sacc. 1889 (H)

Micraspis Darker 1963 (C)

Pragmopora A. Massal $1855(\mathbf{C})^{\#}$

Tympanis Tode 1790 (C)

Phacidiales genera incertae sedis

Coma Nag Raj \& W.B. Kendr. 1972 (C)

Rhytismatales M.E. Barr ex Minter

Rhytismataceae Chevall.

Hypohelion P.R. Johnst. (C)

Ploioderma Darker. 1967 (C)

Rhytisma Fr. 1818 (C)

Leotiomycetes, families incertae sedis

Amorphothecaceae Parbery 
(H)

Hormoconis Arx \& G.A. de Vries 1973

Myxotrichaceae Currah

Geomyces Traaen 1914 (H)

Gymnostellatospora Udagawa et al. 1993

(C)

Oidiodendron Robak 1932 (H)

Leotiomycetes, genera incertae sedis

Epicladonia D. Hawksw. 1981 (C)

(H)

Exochalara W. Gams \& Hol.-Jech. 1976

$(\mathbf{H})^{\# *}$

Gelatinomyces N. Sanoamuang et al. 2013

(H)

Gorgomyces M. Gönczöl \& Révay 1985

Helicocentralis Sri-indr. et al. 2015 (H)*

Leohumicola N.L. Nick. 2005 (H)

(C) ${ }^{\#}$

Ocotomyces H.C. Evans \& Minter 1985

Tetrachaetum Ingold 1942 (H)

Trullula Ces. 1852 (C)

Class Orbiliomycetes O.E. Erikss. \& Baral

Orbiliales Baral et al.

Orbiliaceae Nannf.

Arthrobotrys Corda 1939 (H)

Dactylella Grove 1884 (H)

Dactylellina M. Morelet 1968 (H)

Drechslerella Subram. 1964 (H)

Gamsylella M. Scholler et al. 1999 (H)

Helicoön Morgan 1892 (H)

Hyalorbilia Baral \& G. Marson 2001 (H)

Orbilia Fr. 1836 (H)

Pseudotripoconidium Z.F. Yu \& K.Q. Zhang 2011 (H)

Retiarius D.L. Olivier 1978 (H)

Vermispora Deighton \& Piroz. 1972 (H)

Orbiliales, genera incertae sedis

Microdochiella Hern.-Restr. \& Crous 2015 (H)*

Class Pezizomycetes sensu O.E. Erikss. \& Winka

Pezizales J. Schröt.

Ascobolaceae Boud. ex Sacc.

Cubonia Sacc. 1889 with papulospora-like asexual morph $\mathbf{( H )}{ }^{\#}$

Caloscyphaceae Harmaja

Caloscypha Boud. 1885 (H)
Chorioactidaceae Pfister

Desmazierella Lib. 1829 (H)

Morchellaceae Reichenb.

Morchella Dill. ex Pers.1794 (H) ${ }^{\#}$

Pezizaceae Dumort.

Chromelosporium Corda 1833 (H)

Glischroderma Fuckel 1870 (C)

Oedocephalum Preuss 1851 (H)

Pyronemataceae Corda

Ascorhizoctonia Chin S. Yang \& Korf 1985 (H)

Heydenia Fresen. 1852 (H)

Micronematobotrys Xiang Sun \& L.D. Guo 2010 (H)

Miladina Svrček $1972(\mathbf{H})^{\#}$

Miladina Svrček 1972 (H)

Trichophaea Boud. 1885 (H)

Rhizinaceae Bonord.

Phymatotrichopsis Hennebert 1973 (H)

Sarcoscyphaceae LeGal ex Eckblad

Sarcoscypha (Fr.) Boud. 1885 (H)

Sarcosomataceae Kobayasi

Conoplea Pers. 1797 (H)

Pezizales, genera incertae sedis

Cephaliophora Thaxt. 1903 (H)

Class Sordariomycetes sensu O.E. Erikss. \& Winka

Subclass Diaportheomycetidae Senan. et al.

Annulatascales D'souza et al.

Annulatascaceae S.W. Wong et al.

Teracosphaeria Réblová \& Seifert 2007

with phialophora-like asexual morph $(\mathbf{H}){ }^{\#}$

Atractosporales H. Zhang et al.

Conlariaceae $\mathrm{H}$. Zhang et al.

Conlarium F. Liu \& L. Cai $2012(\mathbf{H})^{\# *}$

Calosphaeriales M.E. Barr (= Jobellisiales M.J. D'souza \& K.D. Hyde)

Calosphaeriaceae Munk

Calosphaeria Tul. \& C. Tul. $1863(\mathbf{H})^{\# *}$

Togniniella Réblová et al. 2004 (H) 
Pleurostomataceae Réblová et al.

Pleurostoma Tul. \& C. Tul. 1863 (H)

Diaporthales Nannf.

Apiosporopsidaceae Senan. et al.

$(\mathbf{C})^{\# *}$

Apiosporopsis (Traverso) Mariani. 1911

Apoharknessiaceae Senan. et al.

(C)*

Apoharknessia Crous \& S.J. Lee 2004

Lasmenia Speg. 1886 (C)

Asterosporiaceae Senan. et al.

Asterosporium Kunze 1819 (C)*

Auratiopycnidiellaceae Senan. et al.

Auratiopycnidiella Crous \& Summerell $2012(\mathbf{C})^{*}$

Coryneaceae Corda (= Pseudovalsaceae M.E. Barr)

Coryneum Nees $1816(\mathbf{C})^{\#}$

Cryphonectriaceae Gryzenh. \& M.J. Wingf.

Aurantioporthe G. Beier \& R.A. Blanchette $2015(\mathbf{H})^{\# *}$

Aurantiosacculus Dyko \& B. Sutton 1979

(C)*

Aurapex Gryzenh. \& M.J. Wingf. 2006 (C)

Celoporthe Nakab. et al. 2006 (C) (C)*

Chrysofolia Crous \& M.J. Wingf. 2015

Chrysoporthe Gry-zenh. \& M.J. Wingf. 2004 (C)

Cryphonectria Sacc. \& D. Sacc. 1905 (C)

Diversimorbus S.F. Chen \& Jol. Roux $2013(\mathbf{C})^{\# *}$

Foliocryphia Cheew. \& Crous 2010 (C)

Luteocirrhus C.F. Crane et al. 2013 (C)*

Mastigosporella Höhn. 1914 (C)

(C)

Ursicollum Gryzenh. \& M.J. Wingf. 2006

Cytosporaceae Fr. (= Valsaceae Tul. \& C. Tul.)

Cytospora Ehrenb. $1818(\text { C })^{\# *}$

Waydora B. Sutton 1976 (C)

Diaporthaceae Höhn. ex Wehm.

Chaetoconis Clem. 1909 (C)*

Chiangraiomyces Senan. \& K.D. Hyde $2017(\mathbf{C})^{\# *}$

Diaporthe Nitschke 1870 (C)
Hyaliappendispora Senan. et al. 2017 (C) ${ }^{\#}$

Mazzantia Mont. 1855 (C) ${ }^{\#}$

Ophiodiaporthe Y.M. Ju et al. 2013 (C)

Phaeocytostroma Petr. 1921 (C)

Pustulomyces D.Q. Dai et al. 2014 (C)*

Stenocarpella Syd. \& P. Syd. 1917 (C)

Erythrogloeaceae Senan. et al.

Chrysocrypta Crous \& Summerell 2012

(C)*

Disculoides Crous et al. 2012 (C)*

Erythrogloeum Petr. 1953 (C)

Gnomoniaceae G. Winter

Amphiporthe Petr. 1971 (C) ${ }^{\#}$

Apiognomonia Höhn. 1917 (C) ${ }^{\# *}$

Asteroma DC. 1815 (C)

Cryptosporella Sacc. 1877 (C)

Cylindrosporella Höhn. 1916 (C)

Gloeosporidina Petr. 1921 (C)

Millerburtonia Cif. 1951 (C)

Plagiostoma Fuckel 1870 (C)

Sirococcus Preuss 1855 (C)

Zythia Fr. 1825 (C)

Harknessiaceae Crous

Harknessia Cooke 1881 (C) ${ }^{\#}$

(C)

Dwiroopa Subram. \& Muthumary 1986

Juglanconidaceae Voglmayr \& Jaklitsch $(\mathbf{C})^{\# *}$

Juglanconis Voglmayr \& Jaklitsch 2017

Lamproconiaceae C. Norphanphoun et al.

Lamproconium (Grove) Grove 1937 (C)*

Macrohilaceae Crous*

Macrohilum H.J. Swart 1988 (C)

Melanconidaceae G. Winter

Melanconis Tul. \& C. Tul. 1863 (C) ${ }^{\#}$

Melanconiellaceae Senan. et al.

Greeneria Scribn. \& Viala 1887 (C)

Melanconiella Sacc. 1882 (C) ${ }^{\#}$

Tubakia B. Sutton 1973 (C)

Prosopidicolaceae Senan. \& K.D. Hyde

Prosopidicola Crous \& C.L. Lennox 2004 (C)*

Pseudoplagiostomaceae Cheew. et al. 
(C) $^{\text {Pseudoplagiostoma Cheew. et al. } 2010}$

Schizoparmeaceae Rossman

Coniella Höhn. 1918 (C)

Stilbosporaceae Link

Crinitospora B. Sutton \& Alcorn 1985

(C)*

Stegonsporium Corda 1827 (C)

Stilbospora Pers. 1794 (C)

Sydowiellaceae Lar. N. Vassiljeva

$(\mathbf{C})^{\# *}$

Breviappendix Senan. \& K.D. Hyde 2017

$(\mathbf{C})^{\# *}$

Hapalocystis Auersw. ex Fuckel 1863

Lambro Racib. 1900 (C) ${ }^{\#}$

Uleoporthe Petr. 1941(C) ${ }^{\#}$ $(\mathbf{C})^{\# *}$

Paragnomonia Senan. \& K.D. Hyde 2017

Tenuiappendicula Senan. et al. $2017(\mathbf{C})^{\# *}$

Tirisporellaceae Suetrong et al.

Thailandiomyces Pinruan et al. $2008(\mathbf{H})^{\#_{*} *}$

Tirisporella E.B.G. Jones et al. $1996(\mathbf{H})^{\# *}$

Diaporthales, genera incertae sedis

Bagadiella Cheew. \& Crous 2009 (H)

Caudospora Starbäck 1889 with phomopsis-like asexual morph (C) ${ }^{\#}$

Gyrostroma Naumov 1914 (C)

Massariovalsa Sacc. 1882 (C)

Melanconiopsis Ellis \& Everh. 1900 (C)

Natarajania Pratibha \& Bhat 2006 (H)

Pachytrype Berl. ex M.E. Barr et al. 1993

with cytospora-like asexual morph $(\mathbf{C})^{\#}$

Rabenhorstia Fr. 1849 (C)

Magnaporthales Thongk. et al.

Magnaporthaceae P.F. Cannon

(H)*

Budhanggurabania P. Wong et al. 2015

Bussabanomyces Klaubauf et al. 2014 (H)*

Clasterosporium Schwein. $1832(\mathbf{H})^{\#}$

Falciphora J. Luo \& N. Zhang 2015 (H)*

Falciphoriella M. Hern.-Restr. \& Crous 2016 (H)*

Gaeumannomycella M. Hern.-Restr. \& Crous 2016 (H)*
Gaeumannomyces Arx \& D.L. Olivier $1952(\mathbf{H})^{\# *}$

Kohlmeyeriopsis Klaubauf et al. 2014 (H) ${ }^{\#}$ $(\mathbf{H})^{\# *}$

Magnaporthiopsis J. Luo \& N. Zhang 2013

Mycoleptodiscus Ostaz. 1968 (H)

Nakataea Hara 1939 (H)

Pseudophialophora J. Luo \& N. Zhang 2014 (H)*

Pyriculariopsis M.B. Ellis 1971 (H)

Slopeiomyces Klaubauf et al. $2014(\mathbf{H})^{\# *}$

Pyriculariaceae Klaubauf et al.

Bambusicularia Klaubauf et al. 2014 (H)*

Barretomyces Klaubauf et al. 2014 (H)*

Deightoniella S. Hughes 1952 (H)

(H)*

Macgarvieomyces Klaubauf et al. 2014

(H)*

Neocordana Hern.-Restr. \& Crous 2015

Neopyricularia Klaubauf et al. 2014 (H)*

Proxipyricularia Klaubauf et al. 2014 (H)*

Pseudopyricularia Klaubauf et al. 2014 (H)*

Pyricularia Sacc. 1880 (H)

Xenopyricularia Klaubauf et al. 2014 (H)*

Myrmecridiales Crous

Myrmecridiaceae Crous

Myrmecridium Arzanlou et al. 2007 (H)

Ophiostomatales Benny \& Kimbr.

Kathistaceae Malloch \& M. Blackw.

Mattirolella S. Colla 1929 (C)

Ophiostomataceae Nannf.

Fragosphaeria Shear 1923 with acremonium-like asexual morph $(\mathbf{H}){ }^{\#}$

Grosmannia Gold. 1936 (H)

Hawksworthiomyces Z.W. de Beer et al. $2016(\mathbf{H})^{*}$

Hyalorhinocladiella H.P. Upadhyay \&

W.B. Kendr. 1975 (H)

Klasterskya Petr. 1940 with hyalorhinocladiella-like asexual morph $(\mathbf{H})^{\#}$

Leptographium Lagerb. \& Melin 1927 (H)

Pesotum J.L. Crane \& Schokn. 1973 (H)

Raffaelea Arx \& Hennebert 1965 (H)

Spumatoria Massee \& E.S. Salmon 1901

(H)

Togniniales I.C. Senanayake et al. 
Togniniaceae Réblová et al. $(\mathbf{H})^{\#}$

Phaeoacremonium W. Gams et al. 1996

Diaportheomycetidae, families incertae sedis

Barbatosphaeriaceae $\mathrm{H}$. Zhang et al.*

Barbatosphaeria Réblová 2007 (H)

Distoseptisporaceae K.D. Hyde \& McKenzie

Distoseptispora K.D. Hyde et al. 2016

(H)*

Lentomitellaceae $\mathrm{H}$. Zhang et al.

Lentomitella Höhn. 1906 (H)

Papulosaceae Winka \& O.E. Erikss.

Platytrachelon Réblová 2013 (H)

Sporidesmiaceae Fr.

Sporidesmium Link $1809(\mathbf{H})^{\# *}$

Thyridiaceae O.E. Erikss \& J.Z. Yue

Pleurocytospora Petr. 1923 (C)

Trichosphaeriaceae G. Winter

Brachysporium Sacc. 1886 (H)

Koorchaloma Subram. 1953 (H)

Stromatographium Höhn. 1907 (H)

Woswasiaceae $\mathrm{H}$. Zhang et al.

Woswasia Jaklitsch et al. $2013(\mathbf{H})^{\# *}$

Xylochrysis Réblová et al. $2014(\mathbf{H})^{\#_{*}}$

Diaportheomycetidae, genera incertae sedis

Aquapteridospora J. Yang et al. 2015 (H)*

Dictyosporella Abdel-Aziz 2015 (H)*

Pseudostanjehughesia J. Yang \& K.D.

Hyde 2017 (H)*

Subclass Hypocreomycetidae O.E. Erikss. \& Winka

Coronophorales Nannf.

Ceratostomataceae G. Winter

Gonatobotrys Corda $1839(\mathbf{H})$

Melanospora Corda 1837 (H)\#

Nitschkiaceae (Fitzp.) Nannf.

Acanthonitschkea Speg. 1908 with acremonium-like asexual morph $(\mathbf{H}){ }^{\#}$

Coronophorales, genera incertae sedis
Pseudocatenomycopsis Crous \& L.A. Shuttlew. 2013 (H)*

Falcocladiales R.H. Perera et al.

Falcocladiaceae Somrithipol et al.

Falcocladium S.F. Silveira et al. 1994 (H)

Glomerellales Chadef. ex Réblová et al.

Australiascaceae Réblová \& W. Gams Monilochaetes Halst. ex Harter 1916 (H)

Glomerellaceae Locq. ex Seifert \& W. Gams Colletotrichum Corda 1831 (C)

Plectosphaerellaceae W. Gams et al. Acrostalagmus Corda 1838 (H)

Chordomyces Bilanenko et al. $2016(\mathbf{H})^{*}$

Gibellulopsis Bat. \& H. Maia 1959 (H)

Lectera P.F. Cannon 2012 (C)*

Longitudinalis Tibpromma \& K.D. Hyde $2017(\mathbf{H})^{*}$

Musicillium Zare \& W. Gams 2007 (H)

Plectosphaerella Kleb. 1929 (H) ${ }^{\#} *$ $(\mathbf{H})^{\# *}$

Sodiomyces Grum-Grzhimaylo et al. 2013

Stachylidium Link 1809 (H)

Verticillium Nees 1816 (H)

Reticulascaceae Réblová \& W. Gams

Blastophorum Matsush. 1971 (H)*

Cylindrotrichum Bonord.1851 (H)

Sporoschismopsis Hol.-Jech. \& Hennebert $1972(\mathbf{H})^{\#}$

Glomerellales genera incertae sedis $(\mathbf{H})^{\# *}$

Malaysiasca Crous \& M.J. Wingf. 2016

Hypocreales Lindau

Bionectriaceae Samuels \& Rossman

Acremonium Link 1809 (H)

Bullanockia Crous 2016 (H)*

Clonostachys Corda 1839 (H)

Dimerosporiella Speg. 1908 with acremonium-like asexual morph $(\mathbf{H})^{\#}$

Fusariella Sacc. 1884 (H)

Gliomastix Guég. 1905 (H)

Gracilistilbella Seifert 2000 (H)

Heleococcum C.A. Jørg. 1922 with trichothecium-like asexual morph $(\mathbf{H})^{\#}$

Hydropisphaera Dumont 1822 with acremonium-like asexual morph (H) 
Ijuhya Starbäck 1899 with acremoniumlike asexual morph $\mathbf{( H )}{ }^{\#}$

Lasionectria (Sacc.) Cooke 1884 with acremonium-like asexual morph (H) ${ }^{\#}$

Mycoarachis Malloch \& Cain 1970 with acremonium-like asexual morph $(\mathbf{H}){ }^{\#}$

Mycocitrus Moller 1901 with acremoniumlike asexual morph (H) ${ }^{\#}$

Nectriella Nitschke ex Fuckel 1870 with acremonium-like and dendrodochium-like asexual morph $\mathbf{( H )}{ }^{\#}$

Nectriopsis Maire 1911 with acremoniumlike asexual morph (H) ${ }^{\#}$

Nectriopsis Maire 1911 with verticilliumlike asexual morph of $\mathbf{( H )}{ }^{\#}$

Nigrosabulum Malloch \& Cain 1970 with acremonium-like asexual morph $(\mathbf{H}){ }^{\#}$

(H)*
Protocreopsis Yoshim. Doi 1977 with

Paracylindrocarpon Crous et al. 2016 acremonium-like asexual morph (H) ${ }^{\#}$

Roumegueriella Speg. 1880 with gliocladium-like asexual morph $(\mathbf{H}){ }^{\#}$

Selinia P. Karst. 1876 with acremoniumlike asexual morph $\mathbf{( H )}{ }^{\#}$

Selinia P. Karst. 1876 with tubercularialike asexual morph (H) ${ }^{\#}$

Spicellum Nicot \& Roquebert 1976 (H)

Stilbocrea Pat. 1900 with acremonium-like asexual morph (H) ${ }^{\#}$

Synnemellisia N.K. Rao et al. 1989 (H)

Verrucostoma Hirooka et al. 2010 with acremonium-like asexual morph (H) ${ }^{\#}$

Clavicipitaceae (Lindau) Earle ex Rogerson

Aciculosporium I. Miyake 1908 (C)

Aphanocladium W. Gams 1971 (H)

Collarina Giraldo et al. 2014 (H)*

Corallocytostroma Y.N. Yu \& Z.Y. Zhang 1980 (C)

Diploöspora Grove 1916 (H)

Flammocladiella Crous et al. 2015 (H)*

Hypocrella Sacc. 1878 (C) ${ }^{\#}$ $(\mathbf{H})^{\# *}$

Metacordyceps G.H. Sung et al. 2007

Metapochonia Kepler et al. 2014 (H)*

Metarhiziopsis D.W. Li et al. 2008 (H)

Metarhizium Sorokīn 1879 (H)

Moelleriella Bres. $1897(\mathbf{C})^{\# *}$

Mycophilomyces Crous \& M.J. Wingf. $2016(\mathbf{H})^{*}$
Neobarya Lowen 1986 with acremoniumlike asexual morph $\mathbf{( H )}{ }^{\#}$

Neotyphodium Glenn et al. 1996 (H)

Nomuraea Maubl. 1903 (H)

Pochonia Bat. \& O.M. Fonseca 1965 (H)

Pseudogibellula Samson \& H.C. Evans

1973 (H)

Rotiferophthora G.L. Barron 1991 (H)

Sphacelia Lév. 1827 (H)

Ustilaginoidea Bref. 1895 (H)

Cordycipitaceae Kreisel ex G.M. Sung et al.

Akanthomyces Lebert 1858 (H)

Amphichorda Fr. 1825 (H)

Beauveria Vuill. 1912 (H)

Engyodontium de Hoog 1978 (H)

Gibellula Cavara 1894 (H)

(H)

Granulomanus de Hoog \& Samson 1978

Isaria Pers. 1794 (H)

Lecanicillium W. Gams \& Zare 2001 (H)

Microhilum H.Y. Yip \& A.C. Rath 1989

(H)

Simplicillium W. Gams \& Zare 2001 (H)

Hypocreaceae De Not.

Aphysiostroma Barrasa et al. 1986 with verticillium-like asexual morph $\mathbf{( H )}{ }^{\#}$

Arachnocrea Z. Moravec 1956 (C)

Cladobotryum Nees 1816 (H)

Cosmospora Rabenh. 1862 with verticillium-like asexual morph (H) ${ }^{\#}$

Escovopsioides H.C. Evans \& J.O. Augustin $2012(\mathbf{H})^{\# *}$

Escovopsis J.J. Muchovej \& Della Lucia 1990 (H)

Hypomyces (Fr.) Tul. \& C. Tul. 1860 with trichothecium-like asexual morph $(\mathbf{H})^{\#}$

Mycogone Link 1809 (H)

Sarawakus Lloyd 1924 with gliocladiumlike and trichoderma-like asexual morph $(\mathbf{H})^{\#}$

Sepedonium Link 1809 (H)

Sibirina G.R.W. Arnold 1970 (H)

Sphaerostilbella (Henn.) Sacc. \& D. Sacc. 1905 (H)

Stephanoma Wallr. 1833 (H)

Trichoderma Pers. 1794 (H)

Verticimonosporium Matsush. 1971 (H)

Nectriaceae Tul. \& C. Tul. $(\mathbf{H})^{\# *}$ 
Atractium Link 1809 (H)

Bisifusarium L. Lombard et al. $2015(\mathbf{H})^{\# *}$

Calonectria De Not. 1867 (H)

Calostilbe Sacc. \& Syd. 1902 (H)

Campylocarpon Halleen et al. 2005

Chaetopsina Rambelli 1956 (H)

Coccinonectria L. Lombard \& Crous 2015 $(\mathbf{H})^{\# *}$

Corallomycetella Henn. 1904 (H)

Corallonectria C. Herrera \& P. Chaverri 2013 (H)

Curvicladiella Decock \& Crous 2006 (H)

Cyanochyta Höhn. 1915 (C)

Cyanophomella Höhn. 1918 (C)

Cylindrocarpostylus R. Kirschner \& Oberw. 1999 (H)*

Cylindrocladiella Boesew. $1982(\mathbf{H})^{\#}$

Cylindrodendrum Bonord. 1851 (H)*

Dacryoma Samuels 1988 (H) $(\mathbf{H})^{\# *}$

Dactylonectria L. Lombard \& Crous 2014

Dematiocladium Allegr. et al. 2005 (H)

Fusarium Link 1809 (H)

Fusicolla Bonord. 1851 (H)*

Gliocephalotrichum J.J. Ellis \& Hesselt. $1962(\mathbf{H})^{\#}$

Gliocladiopsis S.B. Saksena 1954 (H)

Macroconia (Wollenw.) Gräfenhan et al.

(H)

Mariannaea G. Arnaud ex Samson 1974

(H)

Microcera Desm. 1848 (H)

Nalanthamala Subram. 1956 (H) ${ }^{\#}$

Nectria (Fr.) Fr. 1849 (H)

Neonectria Wollenw. 1917 (H)

Hypomyces (Fr.) Tul. \& C. Tul. 1860 with papulaspora-like asexual morph $(\mathbf{H})^{\#}$

Paracremonium L. Lombard \& Crous $2015(\mathbf{H})^{\# *}$

Penicillifer Emden 1968 (H)

Pleiocarpon L. Lombard \& D. Aiello 2017 (H)*

Pleonectria Sacc. $1876(\mathbf{H})^{\#}$

Pseudocosmospora C. Herrera \& P. Chaverri $2013(\mathbf{H})^{\# *}$ $(\mathbf{H})^{\# *}$

Rectifusarium L. Lombard et al. 2015 (H) ${ }^{\#}$

Rugonectria P. Chaverri \& Samuels 2011

Sarcopodium Ehrenb. 1818 (H)

Thyronectria Sacc. 1875 (C)
$(\mathbf{H})^{\# *}$

Varicosporella Lechat \& J. Fourn. 2015

Volutella Fr. 1832 (H)

Xenoacremonium L. Lombard \& Crous $2015(\mathbf{H})^{\# *}$

$(\mathbf{H})^{\#}$

Xenocylindrocladium Decock et al. 1997

Xenoleptographium Marinc. et al. 2015 (H)*

Niessliaceae Kirschst.

Eucasphaeria Crous 2007 (C)

Myrtacremonium Crous 2017 (H)*

Niesslia Auersw. 1869 (H)

Trichosphaerella E. Bommer et al. 1891

with acremonium-like asexual morph $(\mathbf{H}){ }^{\#}$

Valetoniellopsis Samuels \& M.E. Barr 1998 with acremonium-like asexual morph (H)

Ophiocordycipitaceae G.H. Sung et al.

Drechmeria W. Gams \& H.B. Jansson (H)

Elaphocordyceps G.H. Sung \& Spatafora 2007 with verticillium-like asexual morph (H) ${ }^{\#}$

Harposporium Lohde 1874 (H) ${ }^{\#}$

Ophiocordyceps Petch 1931 (H)

Polycephalomyces Kobayasi 1941 (H)

Purpureocillium Luangsa-ard et al. 2011

(H)

Tolypocladium W. Gams 1971 (H)

Stachybotryaceae L. Lombard \& Crous

(H)*

Achroiostachys L. Lombard \& Crous 2016

Albifimbria L. Lombard \& Crous 2016 (H)*

Albosynnema E.F. Morris 1967 (H)

Alfaria Crous et al. $2014(\mathbf{C})^{\# *}$

Alfariacladiella Crous \& R.K. Schumach.

$2016(\mathbf{H})^{*}$

(H)*

Brevistachys L. Lombard \& Crous 2016

Capitofimbria L. Lombard \& Crous 2016

(H)*

(H)*

Cymostachys L. Lombard \& Crous 2016

Didymostilbe Henn. 1902 (H)

Dimorphiseta L. Lombard \& Crous 2016

(H)*

Globobotrys L. Lombard \& Crous 2016

(H)*

Grandibotrys L. Lombard \& Crous 2016 (H)* 
Gregatothecium L. Lombard \& Crous $2016(\mathbf{H})^{*}$ (H)*

Inaequalispora L. Lombard \& Crous 2016

Kastanostachys L. Lombard \& Crous 2016 $(\mathbf{H})^{\# *}$

Koorchalomella Chona et al. 1958 (H)*

Melanopsamma Niessl 1876 (H)

Memnoniella Höhn. 1923 (H)

Myrothecium Tode 1790 (H)

Myxospora L. Lombard \& Crous 2016 (H)*

Neomyrothecium L. Lombard \& Crous 2016 (H)*

Paramyrothecium L. Lombard \& Crous $2016(\mathbf{H})^{*}$

Parasarcopodium Mel'nik et al. 2004 (H)

Parvothecium L. Lombard \& Crous 2016 (H)*

Septomyrothecium Matsush. 1971 (H)

Sirastachys L. Lombard \& Crous 2016 (H)* (H)*

Smaragdiniseta L. Lombard \& Crous 2016

Stachybotrys Corda $1837(\mathbf{H})^{\#}$ $(\mathbf{H})^{\# *}$

Striatibotrys L. Lombard \& Crous 2016

Striaticonidium L. Lombard \& Crous 2016 (H)*

Tangerinosporium L. Lombard \& Crous $2016(\mathbf{H})^{*}$

Virgatospora Finley 1967 (H)

Xenomyrothecium L. Lombard \& Crous 2016 (H)*

Xepicula Nag Raj 1993 (C)

Xepiculopsis Nag Raj 1993 (C)

Tilachlidiaceae Lombard \& Crous

Septofusidium W. Gams 1971 (H)

Tilachlidium Preuss 1851 (H)

Hypocreales, genera incertae sedis

Acremoniopsis Giraldo et al. 2014 (H)*

Cylindrium Bonord. 1851 (H)*

Emericellopsis J.F.H. Beyma 1940 (H) ${ }^{\# *}$

Geosmithia J.Pitt 1979 (H)

Hapsidospora Malloch \& Cain 1970 (C)

Haptospora G.L. Barron 1991 (H)

Harzia Costantin 1888 (H)

Hymenopsis Sacc. 1886 (C)

Illosporiopsis D. Hawksw. 2001 (H)

Illosporium Mart. 1817 (H)
Leucosphaerina Arx 1987 with acremonium-like asexual morph of $(\mathbf{H})^{\#}$

Munkia Speg. 1886 (H)

Neomunkia Petr. 1947 (H)

Neothyronectria Crous \& Thangavel 2016 (C)*

Sarocladium W. Gams \& D. Hawksw. 1976 (H)

Stanjemonium W. Gams et al. 1999 (H)

Stilbella Lindau 1900 (H)

Trichothecium Link 1809 (H)

Melanosporales N. Zhang \& M. Blackw.

Ceratostomataceae G. Winter

Ambrosiella Brader ex Arx \& Hennebert 1965 (H)

Ceratocystis Ellis \& Halst. 1890 (H)\#

Chalaropsis Peyronel 1916 (H)\#

Davidsoniella Z.W. de Beer et al. 2014

(H) \#*

Huntiella Z.W. de Beer et al. 2014 (H)\#*

Thielaviopsis Went 1893 (H)

Chadefaudiellaceae Faurel \& Schotter ex Benny \& Kimbr.

Faurelina Locq.-Lin. 1975 with arthrographis-like asexual morph (H) ${ }^{\#}$

Gondwanamycetaceae Réblová et al.

Custingophora Stolk et al. 1968 (H)

Graphiaceae De Beer

Graphium Corda 1837 (H)

Halosphaeriaceae E. Müll. \& Arx ex Kohlm.

Cirrenalia Meyers \& R.T. Moore 1960 (H)

Corollospora Werderm. 1922 with clavariopsis-like asexual morph (H) ${ }^{\#}$

Clavatospora Sv. Nilsson ex Marvanová \&

Sv. Nilsson 1971 (H)

Corollospora Werderm. $1922(\mathbf{H})^{\#}$

Halosphaeriopsis T.W. Johnson 1958 (H) ${ }^{\#}$

Sigmoidea J.L. Crane 1968 (H)

Microascaceae Luttr. ex Malloch

Acaulium Sopp 1912 (H)

Brachyconidiellopsis Decock et al. 2004

(H)

Cephalotrichum Link 1809 (H)

Echinobotryum Corda 1831 (H)

Gamsia M. Morelet 1969 (H)

Parascedosporium Gilgado et al. 2007 (H) 
Pseudoscopulariopsis M. Sandoval-Denis et al. $2015(\mathbf{H})^{\#} *$

Rhinocladium Sacc. \& Marchal 1885 (H)

Scedosporium Sacc. ex Castell. \& Chalm. 1919 (H)

Wardomyces F.T. Brooks \& Hansf. 1923

(H)

(H)

Wardomycopsis Udagawa \& Furuya 1978

Yunnania H.Z. Kong 1998 (H)

Microascales genera incertae sedis

Cephalotrichiella Crous 2014 (H)*

Ceratocystis Ellis \& Halst. 1890 with chalara-like asexual morph $(\mathbf{H}){ }^{\#}$

Cornuvesica C.D. Viljoen et al. 2000 with chalara-like asexual morph $(\mathbf{H}){ }^{\#}$

Sphaeronaemella P. Karst. 1884 (H)

Sporendocladia G. Arnaud ex Nag Raj \& W.B. Kendr. 1975 (H)

(H)*

Triadelphia Shearer \& J.L. Crane 1971

Torpedosporales E.B.G. Jones et al.

Juncigenaceae E.B.G. Jones et al.

Moheitospora Abdel-Wahab et al. 2010

Torpedosporaceae E.B.G. Jones \& K.L. Pang

Torpedospora Meyers 1957 (H)\#

Hypocreomycetidae genera incertae sedis

Campylospora Ranzoni 1953 (H)*

Dendroclathra Voglmayr \& G. Delgado

2001 (H)

Subclass Lulworthiomycetidae Dayarathne et al.

Lulworthiales Kohlm.

Lulworthiaceae Kohlm.

Cumulospora I. Schmidt 1985 (H)

Halazoon Abdel-Aziz et al. 2010 (C)

Hydea K.L. Pang \& E.B.G. Jones 2010 (H)

Matsusporium E.B.G. Jones \& K.L. Pang

2010 (H)

Moleospora Abdel-Wahab et al. 2010 (H)

Moromyces Abdel-Wahab et al. 2010 (H)

Subclass Savoryellomycetidae Hongsanan et al.

Conioscyphales Réblová \& Seifert

Conioscyphaceae Réblová \& Seifert
Conioscypha Höhn. 1904 (H)\#

Fuscosporellales J. Yang et al.

Fuscosporellaceae J. Yang et al.

Fuscosporella J. Yang et al. 2016 (H)*

Mucispora J. Yang et al. 2016 (H)*

Parafuscosporella J. Yang \& K.D. Hyde

$2016(\mathbf{H}) *$

Pseudoascotaiwania J. Yang et al. 2016

(H)*

Pleurotheciales Réblová \& Seifert

Pleurotheciaceae Réblová \& Seifert

(H)\#* Anapleurothecium Hern.-Restr. et al. 2017

Melanotrigonum Réblová 2015 (H)\#*

Phaeoisaria Höhn. 1909 (H)*

Pleurotheciella Réblová et al. 2012 (H)\#*

Pleurothecium Höhn. 1919 (H)\#

Sterigmatobotrys Oudem. 1886 (H)

Savoryellales Boonyuen et al.

Savoryellaceae Jaklitsch \& Réblová

Canalisporium Nawawi \& Kuthub. 1989

(H)\#

Monotosporella S. Hughes 1958 (H)

(H) $\#^{*}$

Neoascotaiwania Hern.-Restr. et al. 2017

Subclass Sordariomycetidae O.E. Erikss. \&

Winka

Chaetosphaeriales Huhndorf et al.

Chaetosphaeriaceae Réblová et al.

Adautomilanezia Gusmão et al. 2016 (H)*

Anacacumisporium Y. R. Ma \& X. G.

Zhang 2016 (H)*

Brunneodinemasporium Crous \& R.F.

Castañeda 2012 (C)*

Catenularia Grove 1886 (H)

Chaetosphaeria Tul. \& C. Tul. 1863 with

phialophora-like asexual morph $(\mathbf{H})$ \#

Chaetosphaerides Matsush. 2003

ramichloridium-like asexual morph $(\mathbf{H})$ \#

Chloridium Link 1809 (H) \#

Codinaea Maire 1937 (H)

Conicomyces R. C. Sinclair et al. 1983

(H)*

Craspedodidymum Hol.-Jech. 1972 (H)

Cryptophiale Piroz. 1968 (H)

Dendrophoma Sacc. 1880 (C)*

Dictyochaeta Speg. 1923 (H) 
Dinemasporium Lév. 1846 (C)

Eucalyptostroma Crous \& M.J. Wingf. 2016

(C)*

Exserticlava S. Hughes 1978 (H)

Gonytrichum Nees \& T. Nees 1818 (H)

Hemicorynespora M.B. Ellis 1972 (H)

Infundibulomyces Plaingam et al. 2003 (C)

Kionochaeta P.M. Kirk \& B. Sutton 1986

(H)

Menispora Pers. 1822 (H)\#

Menisporopsis S. Hughes 1952 (H)\#*

Neopseudolachnella A. Hashim. \& Kaz.

Tanaka 2015 (C)*

(H)

Pseudobotrytis Krzemien. \& Badura 1954

Pseudodinemasporium A. Hashim. \& Kaz.

Tanaka 2015 (C)*

Pseudolachnea Ranoj. 1910 (C)

Pseudolachnella Teng 1936 (C)*

Pyrigemmula D. Magyar \& R. Shoemaker

2011 (H)

Rattania Prabhugaonkar \& Bhat 2009 (H)

Sporoschisma Berk. \& Broome 1847 (H)\#

Tainosphaeria F.A. Fernández \& Huhndorf

2005 (H)

Thozetella Kuntze 1891 (H)

1965 (H)

Zanclospora S. Hughes \& W.B. Kendr.

Helminthosphaeriaceae Samuels et al.

Diplococcium Grove 1885 (H)

Endophragmiella B. Sutton 1973 (H)

Ruzenia O. Hilber 2002 with

selenosporella-like asexual morph $(\mathbf{H})$ \#

Chaetosphaeriales genera incertae sedis

Calvolachnella Marinc. et al. 2016 (C)*

Nawawia Marvanová 1980 (H)

Coniochaetales Huhndorf et al. (= Cordanales M.

Hern.-Rest. \& Crous)

Coniochaetaceae Malloch \& Cain

Coniochaeta (Sacc.) Cooke $1887(\mathbf{H}) \#$

Barrina A. W. Ramaley 1997 with

phialophora-like asexual morph $(\mathbf{H})$

Cordana Preuss 1851 (H) \#

Rhexodenticula W.A. Baker \& MorganJones 2001 (H)

Meliolales Gäum. ex D. Hawksw. \& O.E.

Erikss.

Meliolaceae G.W. Martin ex Hansf.

Asteridiella McAlpine 1897 (H)\#*
(H)\#*

Cryptomeliola S. Hughes \& Piroz. 1997

Endomeliola S. Hughes \& Piroz. 1994

(H) \#*

Irenopsis F. Stevens 1927 (H)\#*

Meliola Fr. 1825 (H)\#*

Phyllachorales M.E. Barr

Phyllachoraceae Theiss. \& H. Syd.

Cyclodomus Höhn. 1909 (C)

Diachora Müll. 1893 (C)

Linochora Höhn. 1910 (C)

Mycohypallage B. Sutton 1963 (C)

Pseudothiella Petr. 1928 (C)

Rhodosticta Woron. 1911 (C)

Sordariales Chad. ex D. Hawksw. \& O.E. Erikss.

Chaetomiaceae G. Winter

Botryotrichum Sacc. \& Marchal 1885 (H)

Chaetomidium (Zopf) Sacc. 1882 with phialophora-like asexual morph (H) ${ }^{\#}$

Chaetomium Kunze 1817 with papulaspora-like asexual morph (H) ${ }^{\#}$

Crassicarpon Y. Marín et al. $2015(\mathbf{H})^{\# *}$

Humicola Traaen 1914 (H)

Madurella Brumpt 1905 (H)

Myceliophthora Costantin 1892 (H)

Staphylotrichum J. Mey. \& Nicot 1957 (H) $(\mathbf{H})^{\# *}$

Thermothelomyces Y. Marín et al. 2015

Trichocladium Harz 1871 (H)

Lasiosphaeriaceae Nannf.

Cercophora Fuckel 1870 with phialophora-like asexual morph $(\mathbf{H})^{\#}$

Cladorrhinum Sacc. \& Marchal 1885 (H)

Eosphaeria Höhn. 1917 with phialophoralike asexual morph $\mathbf{( H )})^{\#}$

Lasiosphaeria Ces. \& De Not. 1863 with phialophora-like asexual morph (H)

Mammaria Ces. ex Rabenh. 1854 (H)

Podospora Ces. 1856 with phialophoralike asexual morph $(\mathbf{H})^{\#}$

Sordariaceae G. Winter

Neurospora Shear \& B.O. Dodge 1927 (H)

Sordariales, genera incertae sedis

Ascolacicola Ranghoo \& K.D. Hyde 1998

with trichocladium-like asexual morph $(\mathbf{H})^{\#}$ 
Ascotaiwania Sivan. \& H.S. Chang 1992 with trichocladium-like asexual morph $(\mathbf{H})^{\#}$

Cancellidium Tubaki 1975 (H)

Lasiosphaeris Clem. 1909 (H)

Lunulospora Ingold 1942 (H)

Ramophialophora M. Calduch et al. 2004

(H)

Remersonia Samson \& Seifert 1997 (H)

Ypsilonia Lév. 1846 (H)

Sordariomycetidae families incertae sedis Cephalothecaceae Höhn.

Albertiniella Kirschst. 1936 with acremonium-like asexual morph $(\mathbf{H})$ \# (H)

Phialemonium W. Gams \& McGinnis 1983

Sordariomycetidae, genera incertae sedis

Acrodictys M.B. Ellis 1961 (H)

Myxocephala G. Weber et al. 1989 (H)

Linocarpon Syd. \& P. Syd. 1917 with phialophora-like asexual morph (H)

Rhodoveronaea Arzanlou et al. 2007 (H)

Spadicoides S. Hughes 1958 (H)

Subclass Xylariomycetidae sensu O.E. Erikss. \& Winka

Amphisphaeriales D. Hawksw. \& O.E. Erikss.

Amphisphaeriaceae G. Winter

Amphisphaeria Ces. \& De Not. 1863 (C) ${ }^{\# *}$

Griphosphaerioma Höhn. 1918 (C)

Apiosporaceae K.D. Hyde et al.

Arthrinium Kunze 1817 (C)

Endocalyx Berk. \& Broome 1876 (H)

Scyphospora L.A. Kantsch. 1928 (C)

Beltraniaceae Nann.

Beltrania Penz. 1882 (H)

Beltraniella Subram. 1952 (H)

Hemibeltrania Piroz. 1963 (H)

Parapleurotheciopsis P.M. Kirk 1982 (H)

Porobeltraniella Gusmão 2004 (H)

Pseudobeltrania Henn. 1902 (H)

Subramaniomyces Varghese \& V.G. Rao $1980(\mathbf{H})$

Clypeophysalosporaceae Giraldo \& Crous Bagadiella Cheew. \& Crous 2009 (H)

Neophysalospora Crous \& M.J. Wingf. $2014(\mathbf{C})^{\# *}$

Plectosphaera Theiss. 1917 (H)
Hyponectriaceae Petr.

Discosphaerina Höhn 1917 with hormonema-like asexual morph (H)

Phlogicylindriaceae Senan. \& K.D. Hyde Phlogicylindrium Crous et al. 2006 (H)

Sporocadaceae Corda

Annellolacinia B. Sutton 1964 (C)

Bartalinia Tassi 1900 (C)

Broomella Sacc 1883 (C)

Ciliochorella Syd. 1935 (C)

Discosia Lib. 1837 (C)

Doliomyces Steyaert 1961 (C)

Dyrithiopsis L. Cai et al. 2003 (C)

Hyalotiella Papendorf 1967 (C)

Immersidiscosia Kaz. Tanaka et al. 2010

(C)

Monochaetia (Sacc.) Allesch. 1902 (C)*

Morinia Berl. \& Bres. 1889 (C)

Neopestalotiopsis Maharachch. et al. 2014

(C)*

Neotruncatella Hyang B. Lee \& T.T.T. Nguyen 2016 (C)*

Pestalotiopsis Steyaert 1949 (C)

Pseudopestalotiopsis Maharachch. et al. $2014(\mathbf{C})^{*}$

Robillarda Sacc. 1880 (C)

Sarcostroma Cooke 1871 (C)

Seimatosporium Corda 1833 C) ${ }^{\# *}$

Seiridium Nees 1816 (C)

Truncatella Steyaert 1949 (C)

Zetiasplozna Nag Raj 1993 (C)

Xylariales Nannf.

Castanediellaceae Hern.-Restr. et al.

Castanediella Hern.-Restr. et al. 2015 (H)*

Diatrypaceae Nitschke

Cryptovalsa Ces. \& De Not. ex Fuckel $1870(\mathbf{C})^{\# *}$

Diatrype Fr. $1849(\mathbf{C})^{\#_{*}}$

Graphostromataceae M.E. Barr et al. Biscogniauxia Kuntze 1891 (H)\#* Camillea Fr. 1849 (H)\#

Graphostroma Piroz. 1974 with

nodulisporium-like asexual morph (H)\#

Obolarina Pouzar 1986

rhinocladiella-like asexual morph $(\mathbf{H})$ \# with 
Hypoxylaceae DC

Jackrogersella L. Wendt et al. 2017 (H)\#*

Lopadostomataceae Daranag. \& K.D. Hyde

Creosphaeria Theiss. 1910 with libertella-

like asexual morph (C)\#

Lopadostoma (Nitschke) Traverso 1906

with libertella-like asexual morph (C)\#

Microdochiaceae Hern.-Restr. et al.

Idriella P.E. Nelson \& S. Wilh. 1956 (H)

Microdochium Syd. 1924 (H) ${ }^{\# *}$

Selenodriella R.F. Castañeda \& W.B.

Kendr. 1990 (H)

Polystigmataceae Höhn. ex Nannf.

Polystigma DC. 1815 (C)

Xylariaceae Tul. \& C. Tul. $(\mathbf{H})^{\# *}$

Annulohypoxylon Y.M. Ju et al. 2005

Ascotricha Berk. 1838 (H)

$(\mathbf{H})^{\# *}$

Brunneiperidium Daranagama et al. 2015

Circinotrichum Nees 1816 (H)*

Collodiscula I. Hino \& Katum. 1955 (H)

Coniolariella D. Garcı́ et al. with nodulisporium-like and rhinocladiella-like asexual morph $(\mathbf{H})$ \# (H)

Cryptostroma P.H. Greg. \& S. Waller 1952

Daldinia Ces. \& De Not. 1863 (H)

Emarcea Duong et al. $2004(\mathbf{H})^{\# *}$

Halorosellinia Whalley et al. 2000 with geniculosporium-like asexual morph $(\mathbf{H}){ }^{\#}$

Hypocreodendron Henn. 1897 (H)

Hypoxylon Bull. $1791(\mathbf{H})^{\# *}$

Jumillera J.D. Rogers et al. 1997 with geniculosporium-like and libertella-like asexual morph $(\mathbf{C})^{\#}$ or $(\mathbf{H})^{\#}$

Kretzschmaria Fr. 1849 with geniculosporium-like asexual morph $(\mathbf{H}){ }^{\#}$

Kretzschmariella Viégas 1944 with mirandina-like asexual morph $(\mathbf{H})^{\#}$

Libertella Desm. 1830 (C) $(\mathbf{C})^{\# *}$

Lunatiannulus Daranagama et al. 2015

Nemania Gray $1821(\mathbf{H})^{\#}$

Poronia Willd. $1787(\mathbf{H})^{\#}$

Rhopalostroma D. Hawksw. 1977 with nodulosporium-like asexual morph $(\mathbf{H})^{\#}$

Rosellinia De Not. 1844 (H)

Rostrohypoxylon J. Fourn. \& M. Stadler 2010 with sporothrix-like asexual morph $(\mathbf{H})^{\#}$
Ruwenzoria J. Fourn. et al. 2010 with nodulosporium-like or sporothrix-like asexual morph (H) ${ }^{\#}$

Surculiseries Okane et al. 2001 (H)

Vamsapriya Gawas \& Bhat 2006 (H)*

Virgaria Nees $1816(\mathbf{H}){ }^{\#}$

Xylaria Hill ex Schrank $1789(\mathbf{H})^{\#}$

Xylariales, genera incertae sedis

Anungitea B. Sutton 1973 (H)

Ascotrichella Valldos. \& Guarro 1988 with humicola-like asexual morph (H) ${ }^{\#}$

Barrmaelia Rappaz 1995 with libertella-like asexual morph $(\mathbf{C}) \#$

(H)*

Beltraniopsis Bat. \& J.L. Bezerra 1960

Hadrotrichum Fuckel 1865 (H)

Idriellopsis Hern.-Restr. \& Crous 2015

$(\mathbf{C})^{*}$

Kirstenboschia Quaedvl. et al. 2013 (C)*

(H)*

Paraidriella Hern.-Restr. \& Crous 2015

Polyancora Voglmayr \& C. Yule 2006 (H)

Pseudophloeospora Crous \& R.G. Shivas 2010 (C)

Sporidesmina Subram. \& Bhat 1989 (H)

Synnemadiella Crous \& M.J. Wingf. 2016 (H)*

Synnemapestaloides T. Handa \& Y. Harada 2004 (H)

$(\mathbf{H})^{\# *}$

Tristratiperidium Daranagama et al. 2016

Xyladictyochaeta Hern.-Restr. et al. 2017 (H)*

Xylariomycetidae families incertae sedis

Cainiaceae J.C. Krug*

Cainia Arx \& E. Müll. 1955 (C)

Xylariomycetidae genera incertae sedis

Pseudosporidesmium K.D. Hyde \&

McKenzie 2016 (H)*

Sordariomycetes, orders incertae sedis

Amplistromatales D'souza et al.

Amplistromataceae Huhndorf et al.

Acidothrix Hujslová \& M. Kolařík 2014 (H)*

Amplistroma Huhndorf et al. 2009 with acrodontium-like asexual morph $(\mathbf{H}){ }^{\#}$

Wallrothiella Sacc. 1882 (H) 
Parasympodiellales Hern.-Restr. et al.

Parasympodiellaceae Hern.-Restr. et al. Parasympodiella Ponnappa1975 (H)

Vermiculariopsiellales Hern.-Restr. et al.

Vermiculariopsiellaceae Hern.-Restr. et al.

Vermiculariopsiella Bender 1932 (H)

Xenospadicoidales Hern.-Restr. et al.

Xenospadicoidaceae Hern.-Restr et al.

Pseudodiplococcium Hern.-Restr. et al. $2017(\mathbf{H})^{*}$

Xenospadicoides Hern.-Restr. et al. 2017

(H)*

Sordariomycetes, genera incertae sedis

Botryosporium Corda $1831(\mathbf{H})^{*}$

Calcarisporium Preuss 1851 (H)

Cryptomycella Höhn. 1925 (C)

Didymobotryum Sacc. 1886 (H)

Ellisembia Subram. 1992 (H)

Fantasmomyces DongHyeon Lee et al. $2016(\mathbf{H})^{\# *}$

Hyalotiopsis Punith. 1970 (C)

Liberomyces Pažoutová et al. 2012 (C)*

Melanographium Sacc. 1913 (H)

Neoeriomycopsis Crous \& M.J. Wingf. $2015(\mathbf{H})^{*}$

$(\mathbf{H})^{*}$

Neoidriella Hern.-Restr. \& Crous 2015

Nigrospora Zimm. 1902 (H)

(H)*

Paradiplococcium Hern.-Restr. et al. 2017

Paramicrodochium Hern.-Restr. \& Crous 2015 (H)*

Phialemoniopsis Perdomo et al. 2013 (C)*

Phomatospora Sacc. 1875 with sporothrixlike asexual morph $(\mathbf{H})^{\#}$

Pleurophragmium Costantin 1888 (H)*

Selenosporella G. Arnaud ex MacGarvie 1969 (H)

Stanjehughesia Subram. 1992 (H) ${ }^{\#}$

Tectonidula Réblová 2009 with sporothrixlike and ramichloridium-like asexual morph (H) ${ }^{\#}$

Xenodium Syd. 1935 (H)

Zalerion R.T. Moore \& Meyers 1962 (H)

Xylonomycetes R. Gazis \& P. Chaverri Xylonomycetales R. Gazis \& P. Chaverri Xylonomycetaceae R. Gazis \& P. Chaverri
Symbiotaphrina Kühlw. \& Jurzitza ex W. Gams \& Arx 1980 (C)

Trinosporium Crous \& Decock 2012 (C)*

Xylona Gazis \& P. Chaverri 2012 (C)

ASCOMYCOTA families incertae sedis

Batistiaceae Samuels \& K.F. Rodrigues Batistia Cif. 1958 (H)

Pseudeurotiaceae Malloch \& Cain

Teberdinia Sogonov et al. 2005 (H)

Seuratiaceae Vuill. ex M.E. Barr Atichia Flot. 1850 (H)

ASCOMYCOTA, genera incertae sedis

Abropelta B. Sutton 1986 (C)

Acarella Syd. 1927 (C)

Acarellina Bat. \& H. Maia 1960 (C)

Acaroconium Kocourk. \& D. Hawksw.

2008 (C)

Acarocybe Syd. 1937 (H)

Acarocybella M.B. Ellis 1960 (H)

Acarocybellina Subram. 1992 (H)

Acarocybiopsis J. Mena et al. 1999 (H)

Acaropeltis Petr. 1937 (C)

Acerviclypeatus Hanlin 1990 (C)

Achoropeltis Syd. 1929 (C)

Acleistia Bayl. Ell. 1917 (C)

Acontium Morgan 1902 (H)

Acrodictyella W.A. Baker \& Partr. 2001

(H)

Acrodictyopsis P.M. Kirk 1983 (H)

Acrodontiella U. Braun \& Scheuer 1995

(H)

Acrophialophora Edward 1961 (H)

Acrophragmis Kiffer \& Reisinger 1970

(H)

Acrospeira Berk. \& Broome 1857 (H)

Acrostaurus Deighton \& Piroz. 1972 (H)

Actinocladium Ehrenb. 1819 (H)

Actinotexis Arx 1960 (C)

Actinothecium Ces. 1854 (C)

Actinothyrium Kunze 1823 (C)

Acumispora Matsush. 1980 (H)

Aenigmatospora R.F. Castañeda et al. 1999

(H)

Agaricodochium X.J. Liu 1981 (H)

Agarwalomyces R.K. Verma \& Kamal

1987 (H)

Agrabeeja Subram. 1995 (H)

Agyriella Sacc. 1884 (H) 
Agyriellopsis Höhn. 1903 (C)

Ahmadia Syd. 1939 (C)

Ajrekarella Kamat \& Kalani 1964 (C)

Alatosessilispora K. Ando \& Tubaki 1984

(H)

Alciphila Harmaja 2002 (H)

Algonquinia R.F. Castañeda \& W.B.

Kendr. 1991 (H)

Alloneottiosporina Nag Raj 1993 (C)

Allothyriella Bat. et al. 1959 (C)

Allothyrina Bat. \& J.L. Bezerra 1964 (C)

Allothyriopsis Bat. et al. 1959 (C)

Alpakesa Subram. \& K. Ramakr. 1954 (C)

Alpakesiopsis Abbas et al. 2003 (C)

Alveariospora Meir. Silva et al. 2012 (H)*

Alveophoma Alcalde 1952 (C)

Alysidiopsis B. Sutton 1973 (H)

Amallospora Penz. 1897 (H)

Amblyosporium Fresen. 1863 (H)

Ameroconium U. Braun \& Zhurb. 2013

(H)*

Amerodiscosiella M.L. Farr 1961 (C)

Amerodiscosiellina Bat. \& Cavalc. 1966

(C)

Amerosporiopsis Petr. 1941 (C)

Amerosporium Speg. 1882 (C)

Amerosympodula Matsush. 1996 (H)

Amoenodochium Peláez \& R.F. Castañeda

1996 (H)

Amoenomyces R.F. Castañeda et al. 1996

(H)

Amphichaetella Höhn. 1916 (H)

Amphichordia Fr. 1825 (H)

Amphophialis R.F. Castañeda et al. 1998

(H)

Amphoropycnium Bat. 1963 (C)

Ampullicephala R.F. Castañeda et al. 2009

(H)

Ampulliferina B. Sutton 1969 (H)

Anabahusakala Carmo et al. 2014 (H)*

Anacoronospora J.S. Monteiro et al. 2016

(H)*

Anacraspedodidymum C.R. Silva et al. 2014 (H)*

Anaexserticlava T.S. Santa Izabel et al. 2015 (H)*

Anaphysmene Bubák 1906 (C)

Anarhyma M.H. Pei \& Z.W. Yuan 1986

(C)

Anaselenosporella Heredia et al. 2010 (H)

Anaseptoidium R.F. Castañeda et al. 2012

(H)*
Anaverticicladus P.O. Costa et al. 2016 (H)*

Ancoraspora Mig. Rodr. 1982 (H)

Angiopomopsis Höhn. 1912 (C)

Angulimaya Subram. \& Lodha 1964 (H)

Angulospora Sv. Nilsson 1962 (H)

Annellodentimyces Matsush. 1985 (H)

Annellodochium Deighton 1969 (H)

Annellophora S. Hughes 1952 (H)

Annellophorella Subram. 1962 (H)

Annellophragmia Subram. 1963 (H)

Annellospermosporella P.R. Johnst. 1999

(H)

(H)

Annellosympodia McTaggart et al. 2007

Antennatula Fr. ex F. Strauss 1850 (H)

Antennopsis R. Heim 1952 (H)

Anthopsis Fil. March. et al. 1977 (H)

Anthracoderma Speg. 1888 (C)

Antromyces Fresen. 1850 (H)

Anulohypha Cif. 1962 (H)

Anungitopsis R.F. Castañeda \& W.B.

Kendr. 1990 (H)

Aoria Cif. 1962 (C)

Aphanofalx B. Sutton 1986 (C)

Apiocarpella Syd. \& P. Syd. 1919 (C)

Apiosporium Kunze 1817 (C)

Apogloeum Petr. 1954 (C)

Apomelasmia Grove 1937 (C)

Aporellula B. Sutton 1986 (C)

Aposporella Thaxt. 1920 (H)

Apostrasseria Nag Raj 1983 (C)

Arachnophora Hennebert 1963 (H)

Arachnospora R.F. Castañeda et al. 2003

(H)

Arborillus Munt.-Cvetk. \& Gómez-Bolea 1998 (H)

Arborispora K. Ando 1986 (H)

Arcuadendron Sigler \& J.W. Carmich. 1976 (H)

Ardhachandra Subram. \& Sudha 1978 (H)

(H)

Argopericonia B. Sutton \& Pascoe 1987

Aristastoma Tehon 1933 (C)

Arthrobotryum Ces. 1854 (C)

Arthrocristula Sigler et al. 1982 (H)

Arthromoniliphora S.S. Silva et al. 2017

(H)*

Arthropycnis Constant. 1992 (C)

Arthrosporium Sacc. 1880 (H)

Arthrowallemia R.F. Castañeda et al. 1998 
Articulophora C.J.K. Wang \& B. Sutton 1982 (H)

Artocarpomyces Subram. 1996 (H)

Asbolisiomyces Bat. \& H. Maia 1961 (C)

Ascochytopsis Henn. 1905 (C)

Ascochytulina Petr. 1922 (C)

Ascospora Mont. 1849 (C)

Ashtaangam Subram. 1995 (H)

Aspilaima Bat. \& H. Maia 1961 (C)

Astelechia Cif. 1962 (H)

Asterinothyriella Bat. \& Cif. 1959 (C)

Asterinothyrium Bat. et al. 1959 (C)

Asteroconium Syd. \& P. Syd. 1903 (C)

Asteroglobulus Brackel 2011 (C)

Asteromyces F. Moreau \& V. Moreau 1962

(H)

Asteroscutula Petr. 1948 (C)

Asterostomopora Bat. \& H. Maia 1960 (C)

Asterostomopsis Bat. et al. 1959 (C)

Asterostomula Theiss. 1916 (C)

Asterostomulina Bat. et al. 1964 (C)

Astronatelia Bat. \& H. Maia 1962 (C)

Atractilina Dearn. \& Barthol. 1924 (H)

Atrogeniculata J.S. Monteiro et al. 2014 (H)*

Atrosetiphiale Matsush. 1995 (H)

Atrosynnema J.W. Xia et al. 2016 (H)*

Aurosphaeria Sun J. Lee et al. 2009 (C)

Avesicladiella W.P. Wu et al. 1997 (H)

Avettaea Petr. \& Syd. 1927 (C)

Bachmanniomyces D. Hawksw. 1981 (C)

Bacillopeltis Bat. 1957 (C)

Bactridium Kunze 1817 (H)

Bactrodesmiella M.B. Ellis 1959 (H)

Badarisama Kunwar et al. 1986 (H)

Bahuchashaka Subram. 1978 (H)

Bahugada K.A. Reddy \& Vasant Rao 1984

(H)

(H)

Bahukalasa Subram. \& Chandrash. 1979

Balaniopsis P.M. Kirk 1985 (H)

Balanium Wallr. 1833 (H)

Barbarosporina Kirulis 1942 (C)

Barnettella D. Rao \& P. Rag. Rao 1964

(C)

Basauxia Subram. 1995 (H)

Basifimbria Subram. \& Lodha 1968 (H)

Batistina Peres 1961 (C)

Beauveriphora Matsush 1975 (H)

Beccopycnidium F. Stevens 1930 (C)

Beejadwaya Subram. 1978 (H)

Belemnospora P.M. Kirk 1981 (H)
Bellulicauda B. Sutton 1967 (C)

Beltramono Rashmi Dubey et al. 2011 (H)

Beltraniomyces Monohar et al. 2003 (H)

Beniowskia Racib. 1900 (H)

Benjpalia Subram. \& Bhat 1989 (H)

Bhadradriella Nagaraju et al. 2011 (H)

Bhadradriomyces Sureshk. et al. 2009 (H)

Bharatheeya D'Souza \& Bhat 2002 (H)

Bhatia W.A. Baker \& Morgan-Jones 2009

(H)

Bibanasiella R.F. Castañeda \& W.B. Kendr. 1991 (H)

Bicoloromyces Heuchert et al. 2015 (H)*

Biflagellospora Matsush. 1975 (H)

Biflagellosporella Matsush. 1993 (H)

Bimeris Petr. 1949 (C)

Bioconiosporium Bat. \& J.L. Bezerra 1964

(H)

Biophomopsis Petr. 1931 (C)

Bisbyopeltis Bat. \& A.F. Vital 1957 (C)

Bispora Corda 1837 (H)

Bisporostilbella Brandsb. \& E.F. Morris 1971 (H)

Bisseomyces R.F. Castañeda 1985 (H)

Blastocatena Subram. \& Bhat 1989 (H)

Blastodictys M.B. Ellis 1976 (H)

Blastofusarioides Matsush. 1996 (H)

Blastophorella Boedijn 1937 (H)

Blastophragma Subram. 1995 (H)

Blennoria Moug. \& Fr. 1825 (C)

Blennoriopsis Petr. 1919 (C)

Bleptosporium Steyaert 1961 (C)

Blodgettia Harv. 1858 (H)

Bomplandiella Speg. 1886 (H)

Bostrichonema Ces. 1867 (H)

Botryoderma Papendorf \& H.P. Upadhyay 1969 (H)

Botryodiplodina Dias \& Sousa da Câmara 1954 (C)

Botryomonilia Goos \& Piroz. 1975 (H)

Botryostroma Höhn. 1911 (C)

Brachycephala J.S. Monteiro et al. 2015 (H)*

Brachydesmiella G. Arnaud ex S. Hughes 1961 (H)

(H)

Brachysporiellina Subram. \& Bhat 1989

Brachysporiopsis Yanna et al. 2004 (H)

Braunomyces V.A. Melnik \& Crous 2014

(H)*

Brefeldiopycnis Petr. \& Cif. 1932 (H)

Brencklea Petrak 1923 (C) 
Brevicatenospora R.F. Castañeda et al. 2006 (H)

Briania D.R. Reynolds 1989 (H)

Briosia Cavara 1888 (H)

Brycekendrickia Nag Raj 1973 (C)

Bryophytomyces Cif. 1953 (H)

Bulbilopycnis Matsush. 1996 (C)

Bulbocatenospora R.F. Castañeda \& Iturr. 2000 (H)

Bullaserpens Bat. et al. 1965 (C)

Cacumisporium Preuss 1851 (H)

Caeruleoconidia to Zhurb. \& Pino-Bodas 2017 (C)*

(H)

Calcarispora Marvanová \& Marvan 1963

Calcarisporiella de Hoog 1974 (H)

Calceispora Matsush. 1975 (H)

Callistospora Petr. 1955 (C)

Calocline Syd. 1939 (C)

Calongeomyces D. Hawksw. \& Etayo

2011 (H)

Camaroglobulus Speer 1986 (C)

Camaropycnis E.K. Cash 1945 (C)

Camarosporellum Tassi 1902 (C)

Camarosporiopsis Abbas et al. 2000 (C)

Camposporidium Nawawi \& Kuthub. 1988

(H)

Camptomeris Syd. 1927 (H)

Candelabrum Beverw. 1951 (H)

Candelosynnema K.D. Hyde \& Seifert 1992 (H)

Capitorostrum Bat. 1957 (C)

Capnocheirides J.L. Crane \& S. Hughes 1982 (H)

Capnofrasera S. Hughes 2003 (H)

Capnosporium S. Hughes 1976 (H)

Capsicumyces Gamundí et al. 1979 (H)

Carnegieispora Etayo \& F. Berger 2009

(C)

Carrismyces R.F. Castañeda \& Heredia 2000 (H)

Casaresia Gonz. Frag. 1920 (H)

Castanedaea W.A. Baker \& Partr. 2001 (H)

Catenella Bat. \& Peres 1963 (C)

Catenocuneiphora Matsush. 2003 (H)

Catenophora Luttr. 1940 (C)

Catenophoropsis Nag Raj \& W.B. Kendr. 1988 (C)

Catenospegazzinia Subram. 1991 (H)

Catenosubulispora Matsush. 1971 (H)

Catenosynnema Kodsueb et al. 2007 (H)
Catenulaster Bat. \& C.A.A. Costa 1959

(C)

Catinopeltis Bat. \& C.A.A. Costa 1957 (C)

Catinula Lév. 1848 (C)

(H)*

Cecidiomyces U. Braun \& Zhurb. 2012

Ceeveesubramaniomyces Pratibha et al. 2005 (H)

Ceratocladium Corda 1839 (H)

Ceratophorum Sacc. 1880 (H)

Ceratopycnis Höhn. 1915 (C)

Ceratosporella Höhn. 1923 (H)

Ceratosporium Schwein. 1832 (H)

Cercosperma G. Arnaud ex B. Sutton \&

Hodges 1983 (H)

Ceuthodiplospora Died. 1912 (C)

Ceuthosira Petr. 1924 (C)

Ceuthosporella Petr. \& Syd. 1923 (C)

Chaetendophragmia Matsush. 1971 (H)

Chaetoblastophorum Morgan-Jones 1977

(H)

Chaetochalara B. Sutton \& Piroz. 1965

(H)

Chaetocytostroma Petr. 1920 (C)

Chaetodiplis Clem. 1931 (C)

Chaetodiplodina Speg. 1910(C)

Chaetopeltaster Katum. 1975 (C)

Chaetophiophoma Speg. 1910 (C)

Chaetopsis Grev. 1825 (H)

Chaetopyrena Pass. 1881 (C)

Chaetoseptoria Tehon 1937 (C)

Chalarodendron C.J.K. Wang \& B. Sutton 1984 (H)

Chalarodes McKenzie 1991 (H)

Chantransiopsis Thaxt. 1914 (H)

Characonidia Bat. \& Cavalc. 1965 (C)

Charomyces Seifert 1987 (H)

Chasakopama Manohar. et al. 2009 (H)

Cheilaria Lib. 1830 (C)

Cheiroidea W.A. Baker \& Morgan-Jones 2009 (H)

Cheiromycella Höhn. 1910 (H)

Cheiromyceopsis Mercado \& J. Mena 1988

(H)

(H)

Cheiromyces Berk. \& M.A. Curtis 1857

Cheiromycina B. Sutton 1986 (H)

Cheiropolyschema Matsush. 1980 (H)

Chiastospora Riess 1852 (C)

Chithramia Nag Raj 1988 (C)

Chlamydomyces Bainier 1907 (H) 
Chlamydopsis Hol.-Jech. \& R.F. Castañeda 1986 (H)

Choanatiara DiCosmo 1984 (C)

Choreospora Constant. \& R. Sant. 1987

(H)

Chromosporium Corda 1829 (H)

Chrysachne Cif. 1938 (H)

Chrysalidopsis Steyaert 1961 (C)

Chryseidea Onofri 1981 (H)

Cicinnobella Henn. 1904 (C)

Ciferria Gonz. Frag. 1925 (C)

Ciferriella Petr. 1930 (C)

Ciferrina Petr. 1932 (C)

Ciferriopeltis Bat. \& H. Maia 1965 (C)

Ciferrioxyphium Bat. \& H. Maia 1963 (H)

Ciliochora Höhn. 1919 (C)

Ciliophora Petr. 1929 (C)

Ciliophorella Petr. 1940 (C)

Ciliosporella Petr. 1927 (C)

Circinoconiopsis A. Hern.-Gut. 2013 (H)*

Circinoconis Boedijn 1942 (H)

Cissococcomyces Brain 1923 (H)

Civisubramaniania Vittal \& Dorai 1986

(H)

Cladoconidium Bandoni \& Tubaki 1985

(H)

Cladoniicola Diederich et al. 2001 (C)

Cladosporiella Deighton 1965 (H)

Cladosporiopsis S.C. Ren \& X.G. Zhang 2012 (H)*

Clasteropycnis Bat. \& Cavalc. 1963 (C)

Clathroconium Samson \& H.C. Evans 1982 (H)

Clauzadeomyces Diederich 1994 (H)

Clavariana Nawawi 1976 (H)

Cleistocystis Sousa da Câmara 1931 (C)

Cleistonium Speer 1986 (C)

Cleistophoma Petr. \& Syd. 1927 (C)

Chithramia Nag Raj 1988 (C)

Clypeispora A.W. Ramaley 1991 (C)

Clypeochorella Petr. 1923 (C)

Clypeopatella Petr. 1942 (C)

Clypeophialophora Bat. \& Peres 1962 (C)

Clypeopycnis Petr. 1925 (C)

Clypeoseptoria F. Stevens \& P.A. Young 1925 (C)

Clypeostagonospora Punith. 1981 (C)

Coccogloeum Petr. 1955 (C)

Codonmyces Calat. \& Etayo 1999 (H)

Colemaniella Agnihothr. 1974 (H)

Coleodictyospora Charles 1929 (H)

Coleoseptoria Petr. 1940 (C)
Colispora Marvanová 1988 (H)

Colletoconis de Hoog \& Aa 1978 (C)

Colletosporium Link 1824 (H)

Collostroma Petr. 1947 (C)

Columnodomus Petr. 1941 (C)

Columnothyrium Bubák 1916 (C)

Comatospora Piroz. \& Shoemaker 1971

(C)

Comocephalum Syd. 1939 (C)

Complexipes C. Walker 1979 (H)

Condylospora Nawawi 1976 (H)

Coniambigua Etayo \& Diederich 1995 (C)

(H)

Conioscyphopsis Goh \& K.D. Hyde 1998

Coniothyrina Syd. 1912 (C)

Conjunctospora Udagawa \& Uchiy. 1999

(H)

Conostoma Bat. \& J.L. Bezerra 1965 (C)

Conostroma Moesz 1921 (C)

Consetiella Hol.-Jech. \& Mercado 1982

(H)

Coremiella Bubák \& K. Krieg. 1912 (H)

Cornucopiella Höhn. 1915 (C)

Cornutispora Piroz. 1973 (C)

Cornutostilbe Seifert 1990 (H)

Corynecercospora V.K. Pal et al. 2006 (H)

(H)

Corynesporella Munjal \& H.S. Gill 1961

Corynesporina Subram. 2009 (H)

Corynesporopsis P.M. Kirk 1981 (H)

Costanetoa Bat. \& J.L. Bezerra 1963 (C)

Crandallia Ellis \& Sacc. 1897 (C)

Craneomyces Morgan-Jones et al. 1987

(H)

Creodiplodina Petr. 1957 (C)

Creonecte Petr. 1949 (C)

Creoseptoria Petr. 1937 (C)

Creothyriella Bat. \& C.A.A. Costa 1957

(C)

Cribropeltis Tehon 1933 (C)

Crustodiplodina Punith. 1988 (C)

Cryptoceuthospora Petr. 1921 (C)

Cryptocline Petr. 1924 (C)

Cryptocoryneopsis B. Sutton 1980 (H)

Cryptophialoidea Kuthub. \& Nawawi 1987 (H)

Cryptosporium Kunze 1817 (C)

Cryptumbellata Udagawa \& Uchiy. 1999

(H)

Ctenosporium R. Kirschner 2006 (H)

Cubasina R.F. Castañeda 1986 (H)

Culicidospora R.H. Petersen 1960 (H) 
Culicinomyces Couch et al. 1974 (H)

Curucispora Matsush. 1981 (H)

Curvulariopsis M.B. Ellis 1961 (H)

Cyanopatella Petr. 1949 (C)

Cyclomarsonina Petr. 1965 (C)

Cylindrogloeum Petr. 1941 (C)

Cylindromyces Manohar. et al. 2004 (H)

Cylindrothyrium Maire 1907 (C)

Cylindroxyphium Bat. \& Cif. 1963 (C)

Cystidiella Malan 1943 (Y)

Cystotricha Berk. \& Broome 1850 (C)

Cytodiscula Petr. 1931 (C)

Cytogloeum Petr. 1925 (C)

Cytonaema Höhn. 1914 (C)

Cytoplacosphaeria Petr. 1920 (C)

Cytosphaera Died. 1916 (C)

Cytosporella Sacc. 1884 (C)

Cyttariella Palm 1932 (C)

Dactylifera Alcorn 1987 (H)

Dactylosporium Harz 1871 (H)

Dasysticta Speg. 1912 (C)

Davisiella Petr. 1924 (C)

Dearnessia Bubák 1916 (C)

Deccanodia Singhai 1974 (C)

Deichmannia Alstrup \& D. Hawksw. 1990

(H)

Delortia Pat. \& Gaillard 1888 (H)

Dendrodomus Bubák 1915 (C)

Dendrographiella Agnihothr. 1972 (H)

Dendrographium Massee 1892 (H)

Dendrospora Ingold 1943 (H)

Dendrosporium Plakidas \& Edgerton ex

J.L. Crane 1972 (H)

Dendryphiosphaera Lunghini \& Rambelli 1978 (H)

Dennisographium Rifai 1977 (H)

Denticularia Deighton 1972 (H)

Dentocircinomyces R.F. Castañeda \&

W.B. Kendr. 1990 (H)

(H) ${ }^{\#}$

Descalsia A. Roldán \& Honrubia 1989

Desertella Mouch. 1979 (H)

Desmidiospora Thaxt. 1891 (H)

Dexhowardia J.J. Taylor 1970 (H)

Diademospora B.E. Söderstr. \& Bååth 1979 (H)

Diarimella B. Sutton 1980 (C)

Dichelostroma Bat. \& Peres 1963 (C)

Dicholobodigitus G.P. White \& Illman 1988 (H)

Dichotomophthora Mehrl. \& Fitzp. ex P.N. Rao 1966 (H)
Dichotomophthoropsis M.B. Ellis 1971

(H)

Dichotophora Whitton et al. 2012 (H)

Dictyoaquaphila J.S. Monteiro et al. 2016 (H)*

Dictyoarthrinium S. Hughes 1952 (H)

Dictyocatenulata Finley \& E.F. Morris 1967 (H)

Dictyoceratosporella Y.R. Ma \& X.G. Zhang 2016 (H)*

Dictyodesmium S. Hughes 1951 (H)

Dictyophrynella Bat. \& Cavalc. 1964 (H)

Dictyopolyschema M.B. Ellis 1976 (H)

Dictyorostrella U. Braun 1999 (H)

Dictyospiropes M.B. Ellis 1976 (H)

Didymochaetina Bat. \& J.L. Bezerra 1965

(C)

Didymochora Höhn. 1918 (C)

Didymopsis Sacc. \& Marchal 1885 (H)

Didymosporina Höhn. 1916 (C)

Diedickea Syd. \& P. Syd. 1913 (C)

Digicatenosporium S.M. Leão et al. 2015

(H)*

(H)

Digitodochium Tubaki \& Kubono 1989

Digitomyces Mercado et al. 2003 (H)

Digitopodium U. Braun et al. 2005 (H)

Digitoramispora R.F. Castañeda \& W.B.

Kendr. 1990 (H)

Dimastigosporium Faurel \& Schotter 1965 (C)

Diplocladiella G. Arnaud ex M.B. Ellis 1976 (H)

Diplodinis Clem. 1931 (C)

Diplodinula Tassi 1902 (C)

Diploplenodomus Died. 1912 (C)

Diplosporonema Höhn. 1917 (C)

Diplozythiella Died. 1916 (C)

Discogloeum Petr. 1923 (C)

Discomycetoidea Matsush. 1993 (H)

Discosiella Syd. \& P. Syd. 1912 (C)

Discosiellina Subram. \& K.R.C. Reddy 1972 (C)

Discosporina Höhn. 1927 (C)

Discotheciella Syd. \& P. Syd 1917 (C)

Discozythia Petr. 1922 (C)

Dissitimurus E.G. Simmons et al. 1987 (H)

Distophragmia R.F. Castañeda et al. 2015

(H)*

Ditangifibula G.C. Adams 1996 (H)

Domingoella Petr. \& Cif. 1932 (H)

Dothideodiplodia Murashk. 1927 (C) 
Dothioropsis Riedl 1974 (C)

Drepanospora Berk. \& M.A. Curtis 1875

(H)

Drudeola Kuntze 1891 (C)

Drumopama Subram. 1957 (H)

Dryosphaera Jørg. Koch \& E.B.G. Jones 1989 (C)

Dualomyces Matsush. 1987 (H)

Dwayabeeja Subram. 1958 (H)

Dwayaloma Subram. 1957 (H)

Dwayalomella Brisson et al. 1975 (C)

Dwibahubeeja N. Srivast. et al. 1995 (H)

Dwibeeja Subram. 1995 (H)

Dwiroopella Subram. \& Muthumary 1986

(C)

Ebollia Minter \& Caine 1980 (C)

Echinocatena R. Campb. \& B. Sutton 1977

(H)

Echinochondrium Samson \& Aa 1975 (H)

Echinoconidiophorum Pereira-Carv. \&

Dianese 2009 (H)

Elachopeltella Bat. \& Cavalc. 1964 (C)

Elattopycnis Bat. \& Cavalc. 1964 (C)

Elegantimyces Goh et al. 1998 (H)

Elletevera Deighton 1969 (H)

Ellisembiopsis T.S. Santa Izabel \& Gusmão 2013 (H)*

Elotespora R.F. Castañeda \& Heredia 2010 (H)

Embryonispora G.Z. Zhao 2013 (H)*

Enantioptera Descals 1983 (H)

Endobotrya Berk. \& M.A. Curtis 1874 (C)

Endobotryella Höhn. 1909 (C)

Endoconospora Gjaerum 1971 (H)

Endogenospora R.F. Castañeda et al. 2010

(H)

Endomelanconium Petr. 1940 (C)

Endophragmiopsis M.B. Ellis 1966 (H)

Endoplacodium Petr. 1949 (C)

Endoramularia Petr. 1923 (C)

Endosporoideus W.H. Ho et al. 2005 (H)

Endozythia Petr. 1959 (C)

Enerthidium Syd. 1939 (C)

Engelhardtiella A. Funk 1973 (H)

Enridescalsia R.F. Castañeda \& Guarro 1998 (H)

Enthallopycnidium F. Stevens 1925 (C)

Entoderma Hanula et al. 1991 (C)

Epaphroconidia Calat. \& V. Atienza 1995

(C)

Ephelidium C.W. Dodge \& E.D. Rudolph 1955 (C)
Epiclinium Fr. 1849 (C)

Epicoccospora Budathoki \& S.K. Singh 1995 (H)

Epidermidophyton E. Lang 1879 (H)

Epinephroma Zhurb. 2012 (C)*

Episporogoniella U. Braun 1994 (H)

Epistigme Syd. 1924 (C)

Epithyrium (Sacc.) Trotter 1931(C)

Eriocercospora Deighton 1969 (H)

Eriocercosporella Rak. Kumar et al. 1998

(H)

Eriomycopsis Speg. 1910 (H)

Eriospora Berk. \& Broome 1850 (C)

Ernakulamia Subram. 1996 (H)

Erysiphopsis Speg. 1910 (C)

Esteya J.Y. Liou et al. 1999 (H)

Eustilbum Rabenh. 1864 (H)

Evanidomus Caball. 1941 (C)

Everhartia Sacc. \& Ellis 1882 (H)

Everniicola D. Hawksw. 1982 (C)

Eversia J.L. Crane \& Schokn. 1977 (H)

Excipularia Sacc. 1884 (H)

Exophoma Weedon 1926 (C)

Exosporella Höhn. 1912 (H)

Exosporodiella Ganie et al. 2012 (C)*

Fairmaniella Petr. \& Syd. 1926 (C)

Favostroma B. Sutton \& E.M. Davison 1983 (C)

Feltgeniomyces Dieder. 1990 (H)

Fenestroconidia Calat. \& Etayo 1999 (H)

Fissuricella Pore et al. 1977 (H)

Flabellocladia Nawawi 1985 (H)

Flabellospora Alas. 1968 (H)

Flosculomyces B. Sutton 1978 (H)

Frigidispora K.D. Hyde \& Goh 1999 (C)

Fujimyces Minter \& Caine 1980 (C)

Fuligomyces Morgan-Jones \& Kamal 1984

(H)

Fumagopsis Speg. 1910 (H)

Funicularius K.K. Baker \& Zaim 1979 (H)

Furcaspora Bonar 1965 (C)

Fusamen (Sacc.) P. Karst. 1890 (C)

Fuscophialis B. Sutton 1977 (H)

Fusichalara S. Hughes \& Nag Raj 1973

(H)

Fusisporella Speg. 1911 (H)

Fusticeps J. Webster \& R.A. Davey 1980

(H)

Gampsonema Nag Raj 1975 (C)

Gangliophora Subram. 1992 (H)

Gangliostilbe Subram. \& Vittal 1976 (H)

Garnaudia Borowska 1977 (H) 
Gaubaea Petr. 1942 (C)

Gelatinocrinis Matsush. 1995 (H)

Gelatinopycnis Dyko \& B. Sutton 1979

(C)

Gelatinosporium Peck 1873 (C)

Geminoarcus K. Ando 1993 (H)

Gemmulina Descals \& Marvanová 1999

(H)

Gilmaniella G.L. Barron 1964 (H)

Glaphyriopsis B. Sutton \& Pascoe 1987

(C)

Glioannellodochium Matsush. 1989 (H)

Glioblastocladium Matsush. 1989 (H)

Globoconidiopsis G.F. Sepúlveda et al. 2009 (H)

Globoconidium G.F. Sepúlveda et al. 2009

(H)

Gloeocoryneum Weindlm. 1964 (C)

Gloeodes Colby 1920 (C)

Gloeosporiella Cavara 1892 (H)

Gloiosphaera Höhn. 1902 (H)

Glutinium Fr. 1849 (C)

Goidanichiella G.L. Barron ex W. Gams 2009 (H)

Gonatobotryum Sacc. 1880 (H)

Goniopila Marvanová \& Descals 1985 (H)

Goosiella Morgan-Jones et al. 1986 (H)

Goosiomyces N.K. Rao \& Manohar. 1989

(H)

Grallomyces F. Stevens 1918 (H)

Graphiothecium Fuckel 1870 (H)

Groveolopsis Boedijn 1951 (C)

Guedea Rambelli \& Bartoli 1978 (H)

Guelichia Speg. 1886 (H)

Gymnoxyphium Cif. et al. 1963 (C)

Gyrothrix (Corda) Corda 1842 (H)

Hadronema Syd. \& P. Syd. 1909 (H)

Hadrosporium Syd. 1938 (H)

Halysiomyces E.G. Simmons 1981 (H)

Hansfordia S. Hughes 1951 (H)

Hansfordiopeltis Bat. \& C.A.A. Costa $1956(\mathbf{C})$

Hansfordiopeltopsis M.L. Farr 1986 (C)

Hapalosphaeria Syd. 1908 (C)

Haplariopsis Oudem. 1903 (H)

Haplobasidion Erikss. 1889 (H)

Haplolepis Syd. 1925 (C)

Haptocara Drechsler 1975 (H)

Harmoniella V.N. Boriss. 1981 (H)

Harpographium Sacc. 1880 (H)

Harpostroma Höhn. 1928 (C)

Harpostroma Höhn. 1928 (C)
Hawksworthiana U. Braun 1988 (H)

Heimiodora Nicot 1960 (H)

Helensiella Minter et al. 2015 (H)

Helhonia B. Sutton 1980 (C)

Helicodochium J.S. Monteiro et al. 2014

(H)*

Helicofilia Matsush. 1983 (H)

Helicogoosia Hol.-Jech. 1991 (H)

Helicominopsis Deighton 1960 (H)

Helicorhoidion S. Hughes 1958 (H)

Helicosingula P.S. van Wyk et al. 1985

(H)

Helicothyrium I. Hino \& Katum. 1961 (C)

Helicoubisia Lunghini \& Rambelli 1979

(H)

Heliscella Marvanová 1980 (H)

Heliscina Marvanová 1980 (H)

Helminthosporiomyces G.F. Sepúlveda et

al. 2009 (H)

Hemicorynesporella Subram. 1992 (H)

Hemidothis Syd. \& P. Syd. 1916 (C)

Hemisphaeropsis Petr. 1947 (C)

Hendersoniella Tassi 1900 (C)

Hendersonina E.J. Butler 1913 (C)

Hendersoniopsis Höhn. 1918 (C)

Hendersonula Speg. 1880 (C)

Hendersonulina Petr. 1951 (C)

Henicospora P.M. Kirk \& B. Sutton 1980

(H)

Herposira Syd. 1938 (H)

Herreromyces R.F. Castañeda \& W.B.

Kendr. 1991 (H)

Heterocephalum Thaxt. 1903 (H)

Heteroconidium Sawada 1944 (H)

Heterosporiopsis Petr. 1950 (H)

Hexacladium D.L. Olivier 1983 (H)

Himantia Pers. 1801 (sterile)

Hinoa Hara \& I. Hino 1961 (H)

Hirudinaria Ces. 1856 (H)

Hoehneliella Bres. \& Sacc. 1902 (C)

Holubovaea Mercado 1983 (H)

Homalopeltis Bat. \& Valle 1961 (C)

Hormiactis Preuss 1851 (H)

Hormiscioideus M. Blackw. \& Kimbr. 1979 (H)

Hormocephalum Syd. 1939 (H)

Hormographis Guarro et al. 1986 (H)

Hughesinia J.C. Lindq. \& Gamundí 1970

(H)

Humicolopsis Verona 1977 (H)

Hyalobelemnospora Matsush. 1993 (H) 
Hyalocamposporium Révay \& J. Gönczöl 2007 (H)

(H)

Hyalocephalotrichum Nagaraju et al. 2011

Hyalocladium Mustafa 1977 (H)

Hyalocylindrophora J.L. Crane \& Dumont 1978 (H)

Hyalodictyum Woron. 1916 (C)

Hyalohelicomina T. Yokoy. 1974 (H)

Hyalopleiochaeta R.F. Castañeda et al. 1996 (H)

Hyalosynnema Matsush. 1975 (H)

Hyalothyridium Tassi 1900 (C)

Hyalotiella Papendorf 1967 (C)

Hydrometrospora J. Gönczöl \& Révay 1985 (H)

Hymenella Fr. 1822 (H)

Hymeniopeltis Bat. 1959 (C)

Hymenobactron (Sacc.) Höhn. 1916 (H)

Hymenopsis Sacc. 1886 (C)

Hyphodiscosia Lodha \& K.R.C. Reddy 1974 (H)

Hyphodiscosioides Matsush. 1993 (H)

Hyphopolynema Nag Raj 1977 (H)

Hyphostereum Pat. 1892 (C)

Hyphothyrium B. Sutton \& Pascoe 1989

(H)

(H)

Hyphozyma de Hoog \& M.T. Sm. 1981

Hypocline Syd. 1939 (C)

Hypodermina Höhn. 1916 (C)

Hypogloeum Petr. 1923 (C)

Hysteridium P. Karst. 1905 (C)

Hysterodiscula Petr. 1942 (C)

Hysteropycnis Hilitzer 1929 (C)

Ialomitzia Gruia 1964 (H)

Idiocercus B. Sutton 1967 (C)

Imicles Shoemaker \& Hambl. 2001 (H)

Impudentia Vujanović 2003 (H)

Inesiosporium R.F. Castañeda \& W. Gams 1997 (H)

Inifatiella R.F. Castañeda 1985 (H)

Intercalarispora J.L. Crane \& Schokn. 1983 (H)

Ionophragmium Peres 1961 (C)

Irpicomyces Deighton 1969 (H)

Isaria Pers. 1794 (H)

Isariella Henn. 1908 (H)

Ischnostroma Syd. \& P. Syd. 1914 (H)

Isthmoconidium Etayo \& Fr. Berger 2013

(H)*

Isthmolongispora Matsush. 1971 (H)
Isthmophragmospora Kuthub. \& Nawawi 1992 (H)

Isthmospora F. Stevens 1918 (H)

Isthmotricladia Matsush. 1971(H)

Ityorhoptrum P.M. Kirk 1986 (H)

Iyengarina Subram. 1958 (H)

Jahniella Petr. 1921 (C)

Javonarxia Subram. 1995 (H)

Jayarambhatia J. Pratibha 2013 (H)*

Jerainum Nawawi \& Kuthub. 1992 (H)

Jubispora B. Sutton \& H.J. Swart 1986 (C)

Junctospora Minter \& Hol.-Jech. 1981 (H)

Junewangia W.A. Baker \& Morgan-Jones

2002 (H)

Kalamarospora G. Delgado 2011 (H)

Kalchbrenneriella Diederich \& M.S.

Christ. 2002 (H)

Kaleidosporium Van Warmelo \& B. Sutton

1981 (C)

Kamatella Anahosur 1969 (C)

Kamatia V.G. Rao \& Subhedar 1976 (H)

Kameshwaromyces Kamal et al. 1986 (H)

Karsteniomyces D. Hawksw. 1980 (C)

Kendrickiella K. Jacobs \& M.J. Wingf. 2001 (H)

Ketubakia Kamat et al. 1987 (H)

Kiliophora Kuthub. \& Nawawi 1993 (H)

Kionocephala P.M. Kirk 1986 (H)

Kmetia Bres. \& Sacc. 1902 (H)

Kmetiopsis Bat. \& Peres 1960 (H)

Knemiothyrium Bat. \& J.L. Bezerra 1960

(C)

Kodonospora K. Ando 1993 (H)

Kolletes Kohlm. \& Volkm.-Kohlm. 2005

(H)

Kontospora A. Roldán et al. 1990 (H)

Korunomyces Hodges \& F.A. Ferreira 1981 (H)

Kostermansinda Rifai 1968 (H)

(H)

Kostermansindiopsis R.F. Castañeda 1986

Kramabeeja G.V. Rao \& K.A. Reddy 1981

(H)

Kramasamuha Subram. \& Vittal 1973 (H)

Kreiseliella Braun 1991 (C)

Kumanasamuha P.Rag. Rao \& D. Rao 1964 (H)

Kutilakesa Subram. 1956 (H)

Kyphophora B. Sutton 1991 (C)

Lacellina Sacc. 1913 (H)

Lacellinopsis Subram. 1953 (H)

Laciniocladium Petri 1917 (H) 
Laeviomyces D. Hawksw. 1981 (C)

Lagenomyces Cavalc. \& A.A. Silva 1972

(C)

Lambdasporium Matsush. 1971 (H)

Lambinonia Sérus. \& Diederich 2005 (H)

Laocoön J.C. David 1997 (H)

Lappodochium Matsush. 1975 (H)

Lasiodiplodiella Zambett. 1955 (C)

Lasiophoma Speg. 1918 (C)

Lasiothyrium Syd. \& P. Syd. 1913 (C)

Lasmeniella Petr. \& Syd. 1927 (C)

Latericonis G.V. Rao et al. 1984 (H)

Lateriramulosa Matsush. 1971 (H)

Laterispora Uecker et al. 1982 (H)

Lawalreea Dieder. 1990 (C)

Lecaniocola Brain 1923 (C)

Lecanostictopsis B. Sutton \& Crous 1997

(H)

Lecophagus M.W. Dick 1990 (H)

Leeina Petr. 1923 (C)

Leightoniomyces D. Hawksw. \& B. Sutton 1977 (H)

Lembuncula Cif. 1954 (C)

Lemkea Morgan-Jones \& R.C. Sinclair 1983 (H)

Lepisticola W. Gams 2009 (H)

Leprieurinella Bat. \& H. Maia 1961 (C)

Leptochlamys Died. 1921(C)

Leptodermella Höhn. 1915 (C)

Leptodiscella Papendorf 1969 (H)

Leptomelanconium Petr. 1923 (C)

Leptophyllosticta I.E. Brezhnev 1939 (C)

Leptostromella (Sacc.) Sacc. 1884 (C)

Leptothyrella Sacc. 1885 (C)

Leptothyrina Höhn. 1915 (C)

Leptothyrium Kunze 1823 (C)

Leucodochium Syd. \& P. Syd. 1917 (H)

Leuliisinea Matsush. 1985 (H)

Libertiella Speg. \& Roum. 1880 (H)

Lichenobactridium Diederich \& Etayo 1995 (H)

Lichenodiplisiella S.Y. Kondr. \& Kudratov 2002 (C)

(C)

Lichenohendersonia Calat. \& Etayo 2001

Lichenopuccinia D. Hawksw. \& Hafellner 1984 (H)

Lichenostella Calat. \& Etayo 1999 (H)

Linkosia A. Hern. Gut. \& B. Sutton 1997

(H)

Linochorella Syd. \& P. Syd. 1912 (C)

Linodochium Höhn. 1909 (H)
Listeromyces Penz. \& Sacc. 1901 (H)

Lobatopedis P.M. Kirk 1979 (H)

Loliomyces Maire 1937 (H)

Lomaantha Subram. 1954 (H)

Lomachashaka Subram. 1956 (H)

Luxuriomyces R.F. Castañeda 1988 (H)

Luzfridiella R.F. Castañeda \& W.B. Kendr.

1991 (H)

Lylea Morgan-Jones 1975 (H)

Lysotheca Cif. 1962 (C)

Mackenziella Yanna \& K.D. Hyde 2009

(H)

Macroallantina Speer 1987 (C)

Macrodiplodia Sacc. 1884 (C)

Macrotrichum Grev. 1825 (H)

Mahabalella B. Sutton \& S.D. Patil 1966

(H)

Manginella Bat. \& H. Maia 1961 (C)

Mapletonia B. Sutton 1991 (C)

Margarinomyces Laxa 1930 (H)

Martinellisia V.G. Rao \& Varghese 1977

(H)

Massalongina Bubák 1916 (C)

Massariothea Syd. 1939 (C)

Matsushimaea Subram. 1978 (H)

Matsushimiella R.F. Castañeda \& Heredia

2001 (H)

Matsushimomyces V.G. Rao \& Varghese 1979 (H)

(H)

Medusamyces G.L. Barron \& Szijarto 1990

Megalodochium Deighton 1960 (H)

Megaloseptoria Naumov 1925 (C)

Melanocephala S. Hughes 1979 (H)

Melanophoma Papendorf \& J.W. du Toit 1967 (C)

Melophia Sacc. 1884 (C)

Menidochium R.F. Castañeda \& W.B.

Kendr. 1990 (H)

Mercadinula M. Hernández-Restrepo et al. 2011 (H)

Mercadomyces J. Mena 1988 (H)

Merismella Syd. 1927 (C)

Metadiplodia Syd. 1937 (C)

Metazythia Petr. 1950 (C)

Metazythiopsis M. Morelet 1988 (C)

Microblastosporon Cif. 1930 (H)

Microclava F. Stevens 1917 (H)

Microdiscula Höhn. 1915 (C)

Microdothiorella C.A.A. Costa \& Sousa da Câmara 1955 (C) 
Microhendersonula Dias \& Sousa da Câmara 1952 (C)

Microperella Höhn. 1909 (C)

Micropustulomyces R.W. Barreto 1995 (C)

Microtyle Speg. 1919 (C)

Microxyphiella Speg. 1918 (C)

Microxyphiopsis Bat. 1963 (C)

Milospium D. Hawksw. 1975 (H)*

Mindoa Petr. 1949 (C)

Minimidochium B. Sutton 1969 (H)

Minteriella Heredia et al. 2013 (H)*

Minutoexcipula V. Atienza \& D. Hawksw. 1994 (H)

Minutophoma D. Hawksw. 1981 (C)

Mirandina G. Arnaud ex Matsush. 1975

(H)

Miricatena Punith. \& Spooner 2011 (H)

Mirimyces Nag Raj 1993 (C)

Mixtoconidium Etayo 1995 (C)

Mohgaonidium Singhai 1974 (C)

Monochaetiella E. Castell. 1943 (C)

Monochaetinula Muthumary et al. 1986

(C)

Monochaetopsis Pat. 1931(H)

Monodia Breton \& Faurel 1970 (C)

Monodidymaria U. Braun 1994 (H)

Monodisma Alcorn 1975 (H)

Monostichella Höhn. 1916 (C)

Moralesia Urries 1956 (C)

Morosporium Renault \& Roche 1898 (C)

Morrisographium M. Morelet 1968 (H)

Mucosetospora M. Morelet 1972 (C)

Muiogone Thaxt. 1914 (H)

Muirella R. Sprague 1959 (H)

Murogenella Goos \& E.F. Morris 1965 (H)

Mycelephas R.F. Castañeda 2009 (H)

Mycocentrodochium K. Matsush. \& Matsush. 1996 (H)

Mycoënterolobium Goos 1970 (H)

Mycomyces Wyss-Chod. 1928 (H)

Mycopara Bat. \& J.L. Bezerra 1960 (C)

(C)

Mycospraguea U. Braun \& Rogerson 1993

Mycosticta Höhn. 1918 (C)

Mycosylva M.C. Tulloch 1973 (H)

Mycousteria M.L. Farr 1986 (C)

Myiocoprula Petr. 1955 (C)

Myriellina Höhn. 1915 (C)

Myrmecomyces Jouvenaz \& Kimbr. 1991

(H)

Myrotheciastrum Abbas \& B. Sutton 1988

(H)
(H)

Myxoparaphysella Caball. 1941 (C)

Myxosporella Sacc. 1881 (C)

Myxosporidiella Negru 1960 (C)

Myxostomellina Syd. 1931 (C)

Myxothyriopsis Bat. \& A.F. Vital 1956 (C)

Myxothyrium Bubák \& Kabát 1915 (C)

Naemosphaera P. Karst. 1888 (C)

Naemosphaerella Höhn. 1923 (C)

Nagrajia R.F. Castañeda \& W.B. Kendr. 1991 (C)

Nagrajomyces Mel'nik 1984 (C)

Nakatopsis Whitton et al. 2001 (H)

Nanoschema B. Sutton 1980 (C)

Naothyrsium Bat. 1960 (C)

Necraphidium Cif. 1951 (H)

Nematogonum Desm. 1834 (H)

Nematographium Goid. 1935 (H)

Nemozythiella Höhn. 1925 (C)

Neoalpakesa Punith. 1981 (C)

Neoarbuscula B. Sutton 1983 (H)

Neobarclaya Sacc. 1899 (C)

Neodiplodina Petr. 1954 (C)

Neofuckelia Zeller \& Goodd. 1935 (C)

Neoheteroceras Nag Raj 1993 (C)

Neojohnstonia B. Sutton 1983 (H)

Neoligniella Naumov 1951 (C)

Neomarssoniella U. Braun 1991 (C)

Neomelanconium Petr. 1940 (C)

Neopeltis Syd. 1937 (C)

Neopericonia Kamal et al. 1983 (H)

Neophoma Petr. \& Syd. 1927 (C)

Neoplaconema B. Sutton 1977 (C)

Neopodoconis Rifai 2008 (H)

Neospegazzinia Petr. \& Syd. 1936 (C)

Neottiospora Desm. 1843 (C)

Neozythia Petr. 1958 (C)

Neta Shearer \& J.L. Crane 1971 (H)

Nidulispora Nawawi \& Kuthub. 1990 (H)

Nigrolentilocus R.F. Castañeda et al. 2001

(H)

Nigromacula Etayo 2002 (H)

Nigropuncta D. Hawksw. 1981 (C)

Nosophloea Fr. 1849 (C)

Nothospora Peyronel 1913 (H)

Novozymia W.P. Wu 2005 (H)

Nummospora E. Müll. \& Shoemaker 1964

(C)

Nusia Subram. 1995 (H)

Nyctalospora E.F. Morris 1972 (H)

Nypaella K.D. Hyde \& B. Sutton 1992 (C) 
Obeliospora Nawawi \& Kuthub. 1990 (H)

Obstipipilus B. Sutton 1968 (C)

Octopodotus Kohlm. \& Volkm.-Kohlm. 2003 (C)

Odontodictyospora Mercado 1984 (H)

Oedothea Syd. 1930 (H)

Ojibwaya B. Sutton 1973 (H)

Olpitrichum G.F. Atk. 1894 (H)

Omega B. Sutton \& Minter 1988 (C)

Oncopodium Sacc. 1904 (H)

Oncospora Kalchbr. 1880 (C)

Oncosporella P. Karst. 1887 (C)

Oncostroma Bat. \& Marasas 1966 (C)

Onychophora W. Gams et al. 1984 (H)

Oosporidium Stautz 1931 (Y)

Oothyrium Syd. 1939 (C)

Ophiosira Petr. 1955 (C)

Orbimyces Linder 1944 (H)

Orphanocoela Nag Raj 1989 (C)

Ostracoderma Fr. 1825 (C)

Ostracodermidium Mukerji 1973 (H)

Oswaldina Rangel 1921 (C)

Ovadendron Sigler \& J.W. Carmich. 1976

(H)

Paathramaya Subram. 1956 (H)

Pachycladina Marvanová 1987 (H)

Palawaniopsis Bat. et al. 1959 (C)

Paliphora Sivan. \& B. Sutton 1985 (H)

Papilionospora V.G. Rao \& B. Sutton 1976 (H)

Pappimyces B. Sutton \& Hodges 1975 (H)

Paraaoria R.K. Verma \& Kamal 1987 (C)

Paraarthrocladium Matsush. 1993 (H)

Parablastocatena Y.D. Zhang \& X.G. Zhang 2012 (H)*

(H)

Paraceratocladium R.F. Castañeda 1987

Parachionomyces Thaung 1979 (H)

(H)

Paracostantinella Subram. \& Sudha 1989

Paracryptophiale Kuthub. \& Nawawi 1994 (H)

Paracytospora Petr. 1925 (C)

Paradendryphiopsis M.B. Ellis 1976 (H)

Paradidymobotryum C.J.K. Wang \& B. Sutton 1984 (H)

Paradiplodia Speg. ex Trotter 1931 (C)

Paradischloridium Bhat \& B. Sutton 1985

(H)

Paradiscula Petr. 1941(C)

Paraëpicoccum Matsush. 1993 (H)

Parafulvia Kamal et al. 1983 (H)
Parahaplotrichum W.A. Baker \& Partr. 2001 (H)

Paraharknessia Matsush. 2003 (H)

Parahyalotiopsis Nag Raj 1976 (C)

Paramassariothea Subram. \& Muthumary 1979 (C)

Paramenisporopsis Matsush. 2003 (H)

Parapericonia M.B. Ellis 1976 (H)

Parapericoniella U. Braun et al. 2005 (H)

Paraphaeoisaria de Hoog \& MorganJones 1978 (H)

Parapithomyces Thaung 1976 (H)

Parapyricularia M.B. Ellis 1972 (H)

Pararobillarda Matsush. 1996 (C)

Parasphaeropsis Petr. 1953 (C)

Parastigmatellina Bat. \& C.A.A. Costa 1959 (C)

Paratetraploa M.K.M. Wong \& K.D. Hyde 2002 (H)

Paratomenticola M.B. Ellis 1976 (H)

Paratrichoconis Deighton \& Piroz. 1972

(H)

Paraulocladium R.F. Castañeda 1986 (H)

Paspalomyces Linder 1933 (H)

Patriciomyces D. Hawksw. 2001(H)

Peethasthabeeja P. Rag. Rao 1981 (H)

Pellionella (Sacc.) Sacc. 1902 (C)

Peltasterinostroma Punith. 1975 (C)

Peltasteropsis Bat. \& H. Maia 1959 (C)

Peltistroma Henn. 1904 (C)

Peltistromella Höhn. 1907 (C)

Peltosoma Syd. 1925 (C)

Peltostromellina Bat. \& A.F. Vital 1959

(C)

(C)

Peltostromopsis Bat. \& A.F. Vital 1959

Penzigomyces Subram. 1992 (H)

Perelegamyces R.F. Castañeda \& W.B. Kendr. 1990 (H)

Perizomella Syd. 1927 (C)

Pestalozziella Sacc. \& Ellis ex Sacc. 1882

(C)

Petrakiopsis Subram. \& K.R.C. Reddy 1968 (H)

Peyronelia Cif. \& Gonz. Frag. 1927 (H)

Phacostroma Petr. 1955 (C)

Phacostromella Petr. 1955 (C)

Phaeoblastophora Partr. \& Morgan-Jones 2002 (H)

Phaeocandelabrum R.F. Castañeda et al. 2009 (H)

Phaeodactylium Agnihothr. 1968 (H) 
Phaeodiscula Cub. 1891 (C)

Phaeodomus Höhn. 1909 (C)

Phaeohiratsukaea Udagawa \& Iwatsu 1990 (H)

Phaeoidiomyces Dorn.-Silva \& Dianese 2004 (H)

Phaeolabrella Speg. 1912 (C)

Phaeomonilia R.F. Castañeda et al. 2007

(H)

Phaeomonostichella Keissl. ex Petr. 1941

(C)

Phaeophloeosporella Crous \& B. Sutton 1997 (C)

Phaeophomopsis Höhn. 1917 (C)

Phaeoschizotrichum Silva et al. 2015 (H)*

Phaeosporobolus D. Hawksw. \& Hafellner 1986 (H)

Phaeostalagmus W. Gams 1976 (H)

Phaeothyrium Petr. 1947 (C)

Phaeotrichoconis Subram. 1956 (H)

Phaeoxyphiella Bat. \& Cif. 1963 (C)

Phalangispora Nawawi \& J. Webster 1982

(H)

Phialoarthrobotryum Matsush. 1975 (H)

Phialogeniculata Matsush. 1971 (H)

Phialophaeoisaria Matsush. 1995 (H)

Phialosporostilbe Mercado \& J. Mena 1985 (H)

Phialostele Deighton 1969 (H)

Phialotubus R.Y. Roy \& Leelav. 1966 (H)

Phloeoconis Fr. 1849 (H)

Phloeosporina Höhn. 1924 (C)

Phlyctaeniella Petr. 1922 (C)

Phomachora Petr. \& Syd. 1925 (C)

Phomachorella Petr. 1947 (C)

Phomatosporella Tak. Kobay. \& K. Sasaki 1982 (C)

Phomyces Clem. 1931 (C)

Phragmoconidium G.F. Sepúlveda et al. 2009 (H)

Phragmopeltis Henn. 1904 (C)

Phragmospathula Subram. \& N.G. Nair 1966 (H)

Phragmospathulella J. Mena \& Mercado 1986 (H)

Phragmotrichum Kunze 1823 (C)

Phylloedium Fr. 1825 (H)

Phyllohendersonia Tassi 1902 (C)

Physalidiella Rulamort 1990 (H)

Physalidiopsis R.F. Castañeda \& W.B. Kendr. 1990 (H)

Piggotia Berk. \& Broome 1851 (C)
Pinatubo J.B. Manandhar \& Mew 1996

(H)

Piperivora Siboe et al. 1999 (H)

Piricauda Bubák 1914 (H)

Piricaudilium Hol.-Jech. 1988 (H)

Piricaudiopsis J. Mena \& Mercado 1987

(H)

Pirispora Faurel \& Schotter 1966 (C)

Pirostomella Sacc. 1914 (H)

Pithosira Petr. 1949 (H)

Pittostroma Kowalski \& T.N. Sieber 1992

(C)

Placella Syd. 1938 (C)

Placodiplodia Bubák 1916 (C)

Placomelan Cif. 1962 (C)

Placonema (Sacc.) Petr. 1921 (C)

Placonemina Petr. 1921 (C)

Placosphaerina Maire 1917 (C)

Placothea Syd. 1931 (C)

Placothyrium Bubák 1916 (C)

Plagiostigmella Petr. 1949 (C)

Plasia Sherwood 1981 (C)

Plectonaemella Höhn. 1915 (C)

Plectopeltis Syd. 1927 (C)

Plectophomopsis Petr. 1922 (C)

Plectosira Petr. 1929 (C)

Plectronidiopsis Nag Raj 1979 (C)

Plectronidium Nag Raj 1977 (C)

Plenocatenulis Bat. \& Cif. 1959 (C)

Plenophysa Syd. \& P. Syd. 1920 (C)

Plenotrichopsis Bat. 1961 (C)

Plenotrichum Syd. 1927 (C)

Plenozythia Syd. \& P. Syd. 1916 (C)

Pleocouturea G. Arnaud 1911 (C)

Plesiospora Drechsler 1971 (H)

Pleurocolla Petr. 1924 (H)

Pleurodesmospora Samson et al. 1979 (H)

Pleurodiscula Höhn. 1926 (C)

Pleurodomus Petr. 1934 (C)

Pleuropedium Marvanová \& S.H. Iqbal 1973 (H)

Pleurophomopsis Petr. 1924 (C)

Pleuroplaconema Petr. 1923 (C)

Pleuroplacosphaeria Syd. 1928 (C)

Pleurostromella Petr. 1922 (C)

Pleurotheciopsis B. Sutton 1973 (H)

Pleurothyriella Petr. \& Syd. 1925 (C)

Pleurovularia R. Kirschner \& U. Braun 2002 (H)

Pocillopycnis Dyko \& B. Sutton 1979 (C)

Podoplaconema Petr. 1921 (C)

Podosporiella Ellis \& Everh. 1894 (H) 
Podosporiopsis Jian Ma et al. 2016 (H)*

Podosporium Schwein. 1832 (H)

Poikilosperma Bat. \& J.L. Bezerra 1961

(C)

(H)

Polybulbophiale Goh \& K.D. Hyde 1998

Polychaetella Speg. 1918 (C)

Polycladium Ingold 1959 (H)

Polydesmus Mont. 1845 (H)

Polyetron Bat. \& Peres 1963 (C)

Polylobatispora Matsush. 1996 (H)

Polypaecilum G. Sm. 1961 (H)

Polyrostrata T.P. Devi \& N. Mathur 2009

(C)

Polystomellomyces Bat. 1959 (C)

Polystratorictus Matsush. 1993 (H)

Polysynnema Constant. \& Seifert 1988 (H)

Polytretophora Mercado 1983 (H)

Porocladium Descals 1976 (H)

Poropeltis Henn. 1904 (C)

Porophilomyces U. Braun 2000 (H)

Porosubramaniania Hol.-Jech. 1985 (H)

Porrectotheca Matsush. 1996 (C)

Proboscispora Punith. 1984 (C)

Prosthemiella Sacc. 1881 (H)

Protostegiomyces Bat. \& A.F. Vital 1955

(C)

Protostroma Bat. 1957 (C)

Psammina Sacc. \& M. Rousseau ex E.

Bommer \& M. Rousseau 1891 (C)

Pseudoacrodictys W.A. Baker \& MorganJones 2003 (H)

Pseudoanguillospora S.H. Iqbal 1974 (H)

Pseudoaristastoma Suj. Singh 1979 (C)

Pseudoasperisporium U. Braun 2000 (H)

Pseudobasidiospora Dyko \& B. Sutton 1978 (C)

Pseudocanalisporium R.F. Castañeda \&

W.B. Kendr. 1991 (H)

Pseudocenangium P. Karst. 1886 (C)

Pseudochuppia Kamal et al. 1984 (H)

(H)

Pseudoclathrosphaerina Voglmayr 1997

Pseudoconium Petr. 1969 (C)

Pseudocytoplacosphaeria Punith. \& Spooner 2002 (C)

Pseudocytospora Petr. 1923 (C)

Pseudodichomera Höhn. 1918 (C)

Pseudodidymaria U. Braun 1993 (H)

Pseudodiplodia (P. Karst.) Sacc. 1884 (C)

Pseudodiscula Laubert 1911 (C)
Pseudofuscophialis Sivan. \& H.S. Chang 1995 (H)

Pseudogaster Höhn. 1907 (H)

Pseudographiella E.F. Morris 1966 (H)

Pseudomeria G.L. Barron 1980 (H)

Pseudomicrodochium B. Sutton 1975 (H)

Pseudoneottiospora Faurel \& Schotter

1965 (C)

Pseudopatellina Höhn. 1908 (C)

Pseudopeltistroma Katum. 1975 (C)

Pseudopenidiella Crous \& Koukol 2012

(H)*

Pseudopetrakia M.B. Ellis 1971 (H)

Pseudophloeosporella U. Braun 1993 (C)

Pseudophragmotrichum W.P. Wu et al. 1998 (C)

Pseudopolystigmina Murashk. 1928 (C)

Pseudoramularia Matsush. 1983 (H)

Pseudorhizopogon Kobayasi 1983 (C)

Pseudoschizothyra Punith. 1980 (C)

Pseudosigmoidea K. Ando \& N. Nakam. 2000 (H)

Pseudostegia Bubák 1906 (C)

Pseudothyrium Höhn. 1927 (C)

Pseudotorula Subram. 1958 (H)

Pseudotracylla B. Sutton \& Hodges 1976

(C)

Pseudotrichoconis W.A. Baker \& MorganJones 2001 (H)

Pseudozythia Höhn. 1902 (C)

Pterulopsis Wakef. \& Hansf. 1943 (H)

Pterygosporopsis P.M. Kirk 1983 (H)

Pucciniospora Speg. 1886 (C)

Pulchromyces Hennebert 1973 (H)

Pullospora Faurel \& Schotter 1965 (C)

Pulvinella A.W. Ramaley 2001 (H)

Punctillina Toro 1934 (C)

Pycnidioarxiella Punith. \& N.D. Sharma 1980 (C)

(C)

Pycnidiopeltis Bat. \& C.A.A. Costa 1959

Pycnis Bref. 1881 (C)

Pycnodactylus Bat. et al. 1967 (C)

Pycnodallia Kohlm. \& Volkm.-Kohlm. 2001 (C)

Pycnoharknessia Matsush. 1996 (C)

Pycnomma Syd. 1924 (C)

Pycnomoreletia Rulamort 1990 (C)

Pycnoseynesia Kuntze 1898 (C)

Pycnothera N.D. Sharma \& G.P. Agarwal 1974 (C)

Pycnothyriella Bat. 1952 (C) 
Pycnothyrium Died. 1913 (C)

Pyramidospora Sv. Nilsson 1962 (H)

Pyrenyllium Clem. 1909 (C)

Pyrgostroma Petr. 1951 (C)

Pyripnomyces Cavalc. 1972 (C)

Quadracaea Lunghini et al. 1996 (H)

Quadricladium Nawawi \& Kuthub. 1989

(H)

Quasidiscus B. Sutton 1991 (C)

Queenslandia Bat. \& H. Maia 1959 (C)

Quezelia Faurel \& Schotter 1965 (C)

Radiatispora Matsush. 1995 (H)

Raizadenia S.L. Srivast. 1981 (H)

Ramakrishnanella Kamat \& Ullasa ex

Ullasa 1970 (C)

Ramicapitulum Whitton et al. 2012 (H)

Ramicephala Voglmayr \& G. Delgado 2003 (H)

Ramoconidiifera B. Sutton et al. 1996 (H)

Redbia Deighton \& Piroz. 1972 (H)

Refractohilum D. Hawksw. 1977 (H)

(H)

Repetoblastiella R.F. Castañeda et al. 2010

(H)

Retroconis de Hoog \& Bat. Vegte 1989

Rhabdoclema Syd. 1939 (C)

Rhabdogloeopsis Petr. 1925 (C)

Rhabdostromella Höhn. 1915 (C)

Rhabdostromina Died. 1921 (C)*

Rhexoacrodictys W.A. Baker \& MorganJones 2002 (H)

Rhexoampullifera P.M. Kirk 1982 (H)

Rhexoprolifer Matsush. 1996 (H)

Rhinotrichella G. Arnaud ex de Hoog 1977

(H)

Rhipidocephalum Trail 1888 (H)

Rhizosphaerina B. Sutton 1986 (C)

Rhodesia Grove 1937 (C)

Rhodesiopsis B. Sutton \& R. Campb. 1979

(C)

Rhodosticta Woron. 1911 (C)

Rhodothallus Bat. \& Cif. 1959 (H)

Rhombostilbella Zimm. 1902 (H)

Rhopalocladium Schroers et al. 1999 (H)

(C)

Rhynchodiplodia Briosi \& Farneti 1906

Rhynchomyces Willk. 1866 (H)

Rhynchoseptoria Unamuno 1940 (C)

Rhynchosporina Arx 1957 (H)

Riclaretia Peyronel 1915 (H)

Rileya A. Funk 1979 (C)

Robakia Petr. 1952 (C)
Rogergoosiella A. Hern. et al. 1996 (H)

Roscoepoundia Kuntze 1898 (C)

Rosulomyces S. Marchand \& Cabral 1976

(H)

Rota Bat. et al. 1959 (C)

Ruggieria Cif. \& Montemart. 1958 (C)

Sadasivania Subram. 1957 (H)

Sanjuanomyces R.F. Castañeda \& W.B.

Kendr. 1991 (H)

Santapauinda Subram. 1995 (H)

Saprophragma K.B. Deshp. \& K.S. Deshp. 1966 (H)

Sarcinosporon D.S. King \& S.C. Jong 1975 (H)

Sarcophoma Höhn. 1916 (C)

Sarophorum Syd. \& P. Syd. 1916 (H)

Satchmopsis B. Sutton \& Hodges 1975 (C)

Sativumoides S.C. Ren et al. 2012 (H)*

Scaphidium Clem. 1901 (C)

Scenomyces F. Stevens 1927 (H)

Sceptrifera Deighton 1965 (H)

Schizothyra Bat. \& C.A.A. Costa 1957 (C)

Schizothyrella Thüm. 1880 (C)

Schizothyropsis Bat. \& A.F. Vital 1960 (C)

Schizotrichum McAlpine 1903 (H)

Schroeteria G. Winter 1881 (H)

Schwarzmannia Pisareva 1968 (C)

Scirrhophoma Petr. 1941 (C)

Sclerococcum Fr. 1825 (H)

Sclerographiopsis Deighton 1973 (H)

Sclerographium Berk. 1854 (H)

Scleromeris Syd. 1926 (C)

Sclerophoma Höhn. 1909 (C)

Scleropycnis Syd. \& P. Syd. 1911 (C)

Sclerotiella A.K. Sarbhoy \& A. Sarbhoy 1975 (H)

Sclerozythia Petch 1937 (C)

Scolecobasidiella M.B. Ellis 1971 (H)

Scolecobeltrania Iturr. et al. 2013 (H)*

Scolecodochium K. Matsush. \& Matsush. 1996 (H)

Scolecosporiella Petr. 1921 (C)

Scolecotheca Søchting \& B. Sutton 1997

(C)

Scolecozythia Curzi 1927 (C)

Scoliotidium Bat. \& Cavalc. 1963 (C)

Scopaphoma Dearn. \& House 1925 (C)

Scopulariella Gjaerum 1971 (H)

Scothelius Bat. et al. 1965 (C)

Scutisporus K. Ando \& Tubaki 1985 (H)

Scutopeltis Bat. \& H. Maia 1957 (C)

Scutopycnis Bat. 1957 (C) 
Seimatosporiopsis B. Sutton et al. 1972 (C)

Selenosira Petr. 1957 (C)

Selenosporopsis R.F. Castañeda \& W.B. Kendr. 1991 (H)

Semipseudocercospora J.M. Yen 1983 (H)

Septocytella Syd. 1929 (C)

Septogloeum Sacc. 1880 (C)

Septomyxella (Höhn.) Höhn. 1923 (C)

Septopatella Petr. 1925 (C)

Septosporiopsis W.A. Baker \& MorganJones 2009 (H)

Septosporium Corda $1831(\mathbf{H})$

Septotrullula Höhn. 1902 (H)

Sessiliospora D. Hawksw. 1979 (H)

Setolibertella Punith. \& Spooner 1999 (C)

Setophiale Matsush. 1995 (H)

Setosporella Mustafa \& Abdul-Wahid 1989 (H)

Setosynnema D.E. Shaw \& B. Sutton 1985 (H)

Seychellomyces Matsush. 1981 (H)

Seynesiopsis Henn. 1904 (C)

Shawiella Hansf. 1957 (C)

Sheariella Petr. 1952 (C)

Sheathnema Dubey \& Moonambeth 2014 (H)*

Shivomyces Hosag. 2004 (H)

Siamia V. Robert et al. 2000 (H)

Sigmatomyces Sacc. \& P. Syd. 1913 (H)

Simmonsiella J.L. Crane \& A.N. Mill. $2016(\mathbf{H})^{*}$

Sirexcipula Bubák 1907 (C)

Sirocyphis Clem. 1909 (C)

Sirodochiella Höhn. 1925 (H)

Sirogloea Petr. 1923 (C)

Siroligniella Naumov 1926 (C)

Sirophoma Höhn. 1917 (C)

Siroplacodium Petr. 1940 (C)

Siropleura Petr. 1934 (C)

Siroscyphellina Petr. 1923 (C)

Sirosperma Syd. \& P. Syd. 1916 (C)

Sirosphaera Syd. \& P. Syd. 1913 (C)

Sirosporonaemella Naumov 1951 (C)

Sirothecium P. Karst. 1887 (C)

Sirothyriella Höhn. 1910 (C)

Sirothyrium Syd. \& P. Syd. 1916 (C)

Sirozythia Höhn. 1904 (C)

Sirozythiella Höhn. 1909 (C)

Sitochora H.B.P. Upadhyay 1964 (C)

Slimacomyces Minter 1986 (H)
Soloacrospora W.B. Kendr. \& R.F. Castañeda 1991 (H)

Solosympodiella Matsush. 1971 (H)

Soloterminospora Matsush. 1996 (H)

Spegazzinia Sacc. 1879 (H)

Spermatoloncha Speg. 1908 (H)

Spermochaetella Cif. 1954 (C)

Spermospora R. Sprague 1948 (H)

Spermosporella Deighton 1969 (H)

Sphaeridium Fresen. 1852 (H)

Sphaeriostromella Bubák 1916 (C)

Sphaeriothyrium Bubák 1916 (C)

Sphaeromma H.B.P. Upadhyay 1964 (C)

Sphaeronema Fr. 1815 (C)

Sphaerophoma Petr. 1924 (C)

Sphaerulomyces Marvanová 1977 (H)

Sphondylocephalum Stalpers 1974 (H)

Spicularia Pers. 1822 (H)

Spilodochium Syd. 1927 (H)

Spinulospora Deighton 1973 (H)

Spiralum J.L. Mulder 1975 (H)

Spiropes Cif. 1955 (H)

Spondylocladiella Linder 1934 (H)

Spondylocladiopsis M.B. Ellis 1963 (H)

Sporhaplus H.B.P. Upadhyay 1964 (C)

Sporidesmiopsis Subram. \& Bhat 1989 (H)

Sporoglena Sacc. 1894 (H)

Sporophiala P.Rag. Rao 1970 (H)

Sporophora Luteraan 1952 (H)

Stachybotryella Ellis \& Barthol. 1902 (H)

Stachybotryna Tubaki \& T. Yokoy. 1971

(H)

Stagonopatella Petr. 1927 (C)

Stagonopsis Sacc. 1884 (C)

Stagonosporina Tassi 1902 (C)

Stagonostromella Petr. \& Syd. 1927 (C)

Staheliella Emden 1974 (H)

Stalagmochaetia Cif. \& Bat. 1963 (C)

Stauronema (Sacc.) Syd. et al. 1916 (C)

Stauronematopsis Abbas et al. 2002 (C)

Staurophoma Höhn. 1907 (C)

Stegonsporiopsis Van Warmelo \& B. Sutton 1981 (C)

Stellomyces Morgan-Jones et al. 1987 (H)

Stellopeltis Bat. \& A.F. Vital 1959 (C)

Stellospora Alcorn \& B. Sutton 1984 (H)

Stellothyriella Bat. \& Cif. 1959 (C)

Stemphyliomma Sacc. \& Traverso 1911

(H)

Stenellopsis B. Huguenin 1966 (H)

Stenocephalopsis Chamuris \& C.J.K. Wang 1998 (H) 
Stenocladiella Marvanová \& Descals 1987 (H)

Stenospora Deighton 1969 (H)

Stephembruneria R.F. Castañeda 1988 (H)

Stevensonula Petr. 1952 (C)

Stichospora Petr. 1927 (C)

Stictopatella Höhn. 1918 (C)

Stictosepta Petr. 1964 (C)

Stigmatellina Bat. \& H. Maia 1960 (C)

Stigmella Lév. 1842 (C)

Stigmina Sacc. 1980 (H)

Stigmopeltis Syd. 1927 (C)

Stilbellula Boedijn 1951 (H)

Stilbodendron Syd. \& P. Syd. 1916 (H)

Stilbophoma Petr. 1942 (C)

Stilbothamnium Henn. 1896 (H)

Strasseria Bres. \& Sacc. 1902 (C)

Strasseriopsis B. Sutton \& Tak. Kobay. 1970 (C)

(H)

Stratiphoromyces Goh \& K.D. Hyde 1998

Striosphaeropsis Verkley \& Aa 1997 (C)

Stromatocrea W.B. Cooke 1952 (H)

Stromatopogon Zahlbr. 1897 (C)

Stromatopycnis A.F. Vital 1956 (C)

Stromatostysanus Höhn. 1919 (H)

Strongylothallus Bat. \& Cif. 1959 (H)

Stygiomyces Coppins \& S.Y. Kondr. 1995

(C)

Stylaspergillus B. Sutton et al. 1982 (H)

Subhysteropycnis Wedin \& Hafellner 1998

(C)

Subicularium M.L. Farr \& Goos 1989 (H)

Subramania D. Rao \& P.Rag. Rao 1964

(H)

Subramanianospora Narayanan et al. 2003

(H)

Subulispora Tubaki 1971 (H)

Suttoniella S. Ahmad 1961(C)

Suttonina H.C. Evans 1984 (C)

(H)

Syamithabeeja Subram. \& Natarajan 1976

Sylviacollaea Cif. 1963 (C)

Symphysos Bat. \& Cavalc. 1967 (C)

Sympodiella W.B. Kendr. 1958 (H)

Sympodiocladium Descals 1982 (H)

Sympodioclathra Voglmayr 1997 (H)

Sympodioplanus R.C. Sinclair \& Boshoff

1997 (H)

Sympodiosynnema J.W. Xia \& X.G. Zhang $2016(\mathbf{H})^{*}$
Synchronoblastia Uecker \& F.L. Caruso 1988 (C)

Syncladium Rabenh. 1859 (C)

Synnemacrodictys W.A. Baker \& MorganJones 2009 (H)

Synnemaseimatoides K. Matsush. \& Matsush. 1996 (H)

Synnematium Speare 1920 (H)

Synnematomyces Kobayasi 1981 (H)

Synostomina Petr. 1949 (C)

Systremmopsis Petr. $1923(\mathbf{H})$

Taeniolina M.B. Ellis 1976 (H)

Talekpea Lunghini \& Rambelli 1979 (H)

Talpapellis Alstrup \& M.S. Cole 1998 (H)

Tandonea M.D. Mehrotra 1991 (C)

Tarsodisporus Bat. \& A.A. Silva 1965 (C)

Tandonella S.S. Prasad \& R.A.B. Verma 1970 (H)

Tectacervulus A.W. Ramaley 1992 (C)

Telligia Hendr. 1948 (H)

Temerariomyces B. Sutton 1993 (H)

Teratosperma Syd. \& P. Syd. 1909 (H)

Termitaria Thaxt. 1920 (H)

Termitariopsis M. Blackw et al. 1980 (H)

Tetrabrachium Nawawi \& Kuthub. 1987

(H)

Tetrabrunneospora Dyko 1978 (H)

Tetracoccosporium Szabó 1905 (H)

Tetrameronycha Speg. ex W. Rossi \& M. Blackw. 1990 (H)

Tetranacriella Kohlm. \& Volkm.-Kohlm. 2001 (C)

Tetranacrium H.J. Huds. \& B. Sutton 1964

(C)

Tetraposporium S. Hughes 1951 (H)

Textotheca Matsush. 1996 (C)

Thallospora L.S. Olive 1948 (H)

Thaptospora B. Sutton \& Pascoe 1987 (C)

Tharoopama Subram. 1956 (H)

Thirumalacharia Rathaiah 1981 (H)

Tholomyces Matsush. 2003 (C)

Thoracella Oudem. 1900 (C)

Thrinacospora Petr. 1948 (C)

Thyrinula Petr. \& Syd. 1924 (C)

Thyriostromella Bat. \& C.A.A. Costa 1959

(C)

Thyrostromella Höhn. 1919 (H)

Thyrsidiella Höhn. ex Höhn. 1909 (C)

Thyrsidina Höhn. 1905 (C)

Tiarosporellivora Punith. 1981 (C)

Ticogloea G. Weber et al. 1994 (H) 
Ticosynnema R.F. Castañeda et al. 2013 (H)*

Tilakiopsis V.G. Rao 1994 (H)

Titaea Sacc. 1876 (H)

Titaeopsis B. Sutton \& Deighton 1984 (H)

Titaeospora Bubák 1916 (C)

Tomenticola Deighton 1969 (H)

Tompetchia Subram. 1985 (H)

Toxosporiella B. Sutton 1986 (C)

Toxosporiopsis B. Sutton \& Sellar 1966

(C)

Toxosporium Vuill. 1896 (H)

Tracylla (Sacc.) Tassi 1904 (C)

Trematophoma Petr. 1924 (C)

Tremellidium Petr. 1927 (C)

Tretendophragmia Subram. 1995 (H)

Tretocephala Subram. 1995 (H)

Tretolylea Cantillo et al. 2015 (H)*

Tretophragmia Subram. \& Natarajan 1974

(H)

Tretospeira Piroz. 1972 (H)

Tretovularia Deighton 1984 (H)

Tribolospora D.A. Reid 1966 (C)

Tricellula Beverw. 1954 (H)

Trichaegum Corda 1837 (H)

Trichobolbus Bat. 1964 (C)

Trichobotrys Penz. \& Sacc. 1901 (H)

Trichoconiella B.L. Jain 1976 (H)

Trichoconis Clem. 1909 (H)

Trichodiscula Vouaux 1910 (C)

Trichodochium Syd. 1927 (H)

Trichomatoclava G.F. Sepúlveda et al. 2009 (H)

Trichomatomyces Dorn.-Silva \& Dianese 2004 (H)

Trichomatosphaera Pereira-Carv. et al. 2009 (H)

Trichopeltulum Speg. 1889 (C)

Trichoseptoria Cavara 1892 (C)

Trichosporiella Kamyschko 1960 (H)

Trichosporodochium Dorn.-Silva \& Dianese 2004 (H)

Trichotheca P. Karst. 1887 (H)

Tricladiella K. Ando \& Tubaki 1984 (H)

Tricladiopsis Descals 1982 (H)

Tricladiospora Nawawi \& Kuthub. 1988

(H)

Tricornispora Bonar 1967 (H)

Trifurcospora K. Ando \& Tubaki 1988 (H)

Triglyphium Fresen. 1852 (H)

Trigonosporium Tassi 1900 (C)

Tripoconidium Subram. 1978 (H)
Triposporina Höhn. 1912 (H)

Triramulispora Matsush. 1975 (H)

Triscelophorus Ingold 1944 (H)

Triscelosporium Nawawi \& Kuthub. 1987

(H)

Trisulcosporium H.J. Huds. \& B. Sutton 1964 (H)

Troposporium Harkn. 1884 (H)

Troposporopsis Whitton et al. 1999 (H)

Tryblidiopycnis Höhn. 1918 (C)

Tryssglobulus B. Sutton \& Pascoe 1987

(H)

Tuberculariopsis Höhn. 1909 (H)

Tuberculispora Deighton \& Piroz. 1972

(H)

Tulipispora Révay \& Gönczöl 2009 (H)

Tumularia Descals \& Marvanová 1987(H)

Tunicago B. Sutton \& Pollack 1977 (C)

Turturconchata J.L. Chen et al. 1999 (H)

Tympanosporium W. Gams 1974 (H)

Uberispora Piroz. \& Hodges 1973 (H)

Ubrizsya Negru 1965 (C)

Ulocoryphus Michaelides et al. 1982 (H)

Umbellidion B. Sutton \& Hodges 1975 (H)

Uniseta Ciccar. 1948 (C)

Urohendersonia Speg. 1902 (C)

Urohendersoniella Petr. 1955 (C)

Uvarispora Goos \& Piroz. 1975 (H)

Vagnia D. Hawksw. \& Miądl. 1997 (C)

Vanakripa Bhat et al. 1993 (H)

Vanbeverwijkia Agnihothr. 1961 (H)

Vanderystiella Henn. 1908 (C)

Vanterpoolia A. Funk 1982 (H)

Vasudevella Chona et al. 1957 (C)

Velutipila D. Hawksw. 1987 (H)

Ventrographium H.P. Upadhyay et al. 1986 (H)

Venustocephala Matsush. 1995(H)

Venustosynnema R.F. Castañeda \& W.B.

Kendr. 1990 (H)

Veracruzomyces Mercado et al. 2002 (H)

Veramycella G. Delgado 2009 (H)

Veramyces Matsush. 1993 (H)

Verdipulvinus A.W. Ramaley 1999 (H)

Veronaella Subram. \& K.R.C. Reddy 1975

(C)

Veronidia Negru 1964 (C)

Verrucariella S. Ahmad 1967 (C)

Verrucophragmia Crous et al. 1994 (H)

Verticicladus Matsush. 1993 (H)

Vesicladiella Crous \& M.J. Wingf. 1994

(H) 
(H)

Vesiculohyphomyces Armando et al. 2009

Vestigium Piroz. \& Shoemaker 1972 (C)

Virgariella S. Hughes 1953 (H)

Viscomacula R. Sprague 1951 (H)

Vittalia Gaws \& Bhat 2007 (H)

Vouauxiella Petr. \& Syd. 1927 (C)

Waihonghopes Yanna \& K.D. Hyde 2002

(H)

Wardinella Bat. \& Peres 1960 (C)

Websteromyces W.A. Baker \& Partr. 2000

(H)

Weissia Bat. \& M.P. Herrera 1964 (C)

Weufia Bhat \& B. Sutton 1985 (H)

Wilsonomyces Adask. et al. 1990 (H)

Wojnowicia Sacc. 1899 (C)

Xanthoriicola D. Hawksw. 1973 (H)

Xenidiocercus Nag Raj 1993 (C)

Xenochora Petr. 1948 (C)

Xenodomus Petr. 1922 (C)

Xenoheteroconium Bhat et al. 1993 (H)

Xenokylindria DiCosmo et al. 1983 (H)

Xenopeltis Syd. \& P. Syd. 1919 (C)

Xenoplaca Petr. 1949 (H)

Xenostroma Höhn. 1915 (C)

Xeroconium D. Hawksw. 1981 (C)

Xiambola Minter \& Hol.-Jech. 1981 (H)

Xiphomyces Syd. \& P. Syd. 1916 (H)

Xiuguozhangia K. Zhang et al. 2014 (H)*

Xylochia B. Sutton 1983 (H)

Xyloglyphis Clem. 1909 (C)

Xylohypha (Fr.) E.W. Mason 1960 (H)

Xylohyphopsis W.A. Baker \& Partr. 2000

(H)

Yalomyces Nag Raj 1993 (C)

Yinmingella Goh et al. 1999 (H)

Ypsilina J. Webster et al. 1999 (H)

Ypsilomyces D.A.C. Almeida \& Gusmão

2014 (H)*

Yuccamyces Gour et al. 1979 (H)

Zakatoshia B. Sutton 1973 (H)

Zebrospora McKenzie 1991 (H)

Zelandiocoela Nag Raj 1993 (C)

Zelodactylaria A.C. Cruz et al. 2012 (H)*

Zelopelta B. Sutton \& R.D. Gaur 1984 (C)

Zelosatchmopsis Nag Raj 1991 (C)

Zelotriadelphia R.F. Castañeda et al. 2005

(H)

Zetesimomyces Nag Raj 1988 (C)

Zevadia J.C. David \& D. Hawksw. 1995

(H)

Zilingia Petr. 1934 (C)
Zinzipegasa Nag Raj 1993 (C)

Zopheromyces B. Sutton \& Hodges 1977

(H)

Zunura Nag Raj 1993 (C)

Zygosporium Mont. 1842 (H)

Zythia Fr. 1849 (C)

Zyxiphora B. Sutton 1981 (H)

Phylum BASIDIOMYCOTA R.T. Moore

Subphylum AGARICOMYCOTINA Doweld

Class Agaricomycetes Doweld

Agaricales Underw.

Agaricaceae Chevall.

Coccobotrys Boud. \& Pat. 1900 (H)

Fistulinaceae Lotsy

Confistulina Stalpers 1983 (H)

Lyophyllaceae Jülich

Termitosphaera Cif. 1935 (H)

Mycenaceae Overeem

Decapitatus Redhead \& Seifert 2000 (H)

Niaceae Jülich

Peyronelina P.J. Fisher et al. 1976 (H)

Physalacriaceae Corner

Mycotribulus Nag Raj \& W.B. Kendr. $1970(\mathbf{C})$

Pleurotaceae Kühner

Antromycopsis Pat. \& Trab. 1897 et al. (H)

Nematoctonus Drechsler 1941 et al. (H)

Psathyrellaceae Vilgalys et al.

(H)

Hormographiella Guarro \& Gené 1992

Rhacophyllus Berk. \& Broome 1871 (H)

Tricholomataceae R. Heim ex Pouzar

Moniliophthora H.C. Evans et al. 1978 (H)

Nothoclavulina Singer 1970 (H)

Tilachlidiopsis Keissl. 192 (H)

Tilachlidiopsis Keissl. 1924 (H)

Ugola Adans. 1763(H)

Agaricales, genera incertae sedis

Aleurocystis Lloyd ex G. Cunn. 1956 (C)

Disporotrichum Stalpers 1984

Fibulochlamys A.I. Romero \& Cabral 1989 (H) 
Tricladiomyces Nawawi 1985 (H)

Atheliales Jülich

Atheliaceae Jülich

Digitatispora Doguet 1962 (H)*

Fibularhizoctonia G.C. Adams \& Kropp 1996 (H)

Taeniospora Marvanová 1977 (H)

Auriculariales J. Schröt.

Exidiaceae R.T. Moore

Ovipoculum Zhu L. Yang \& R. Kirschner $2010(\mathbf{H})$

Auriculariales, genera incertae sedis

Disporotrichum Stalpers 1984 (H)

Oliveorhiza P. Roberts 1998 (H)

Porpopycnis R. Kirschner 2012 (C)

Cantharellales Gäum

Botryobasidiaceae Jülich

Haplotrichum Link 1824 (H)

Ceratobasidiaceae G.W. Martin

Acanthellorhiza P. Roberts 1999 (H)

Ceratorhiza R.T. Moore 1987 (H)

Rhizoctonia DC. 1805 (H)

Hydnaceae Chevall.

Burgella Diederich \& Lawrey 2007 (H)

Burgoa Goid. 1938 (H)

Ingoldiella D.E. Shaw 1972 (H)

Osteomorpha G. Arnaud ex Watling \&

W.B. Kendr. 1979 (H)

Oliveoniaceae P. Roberts 1998

Oliveorhiza P. Roberts 1998 (H)

Tulasnellaceae Juel

Epulorhiza R.T. Moore 1987 (H)

Cantharellales, genera incertae sedis

Minimedusa Weresub \& P.M. LeClair $1971(\mathbf{H})$

Corticiales K.H. Larss.

Corticiaceae Herter

Chrysorhiza T.F. Andersen \& Stalpers 1996 (H)

Erythricium J. Erikss. \& Hjortstam 1970 (H)

Giulia Tassi 1904 (C)
Marchandiomyces Dieder. \& D. Hawksw. 1990 (H)

Michenera Berk. \& M.A. Curtis 1868 (H)

Necator Massee 1898 (H)

Tretopileus B.O. Dodge 1946 (H)

Hymenochaetales Oberw.

Schizoporaceae Jülich

Echinodia Pat. 1918 (H)

Hymenochaetales, genera incertae sedis

Caeruleomyces Stalpers 2000 (H)

Polyporales Gäum.

Fomitopsidaceae Jülich

Ptychogaster Corda 1838 (H)

Ganodermataceae Donk

Thermophymatospora Udagawa et al. 1986

(H)

Hyphodermataceae Jülich

Aegerita Pers. 1801 (H)

Aegeritina Jülich 1984 (H)

Meruliaceae P. Karst.

Bornetina L. Mangin \& Viala 1903 (H)

Phanerochaetaceae Jülich

(H)

Erythricium J. Erikss. \& Hjortstam 1970

Sporotrichum Link 1809 (H)

Polyporaceae Fr. ex Corda

Digitellus Paulet 1791 (H)

Mycelithe Gasp. 1841 (C)

Pachyma Fr. 1822 (H)

Russulales Kreisel ex P.M. Kirk et al.

Bondarzewiaceae Kotl. \& Pouzar

Spiniger Stalpers 1974 (H)

Peniophoraceae Lotsy

Licrostroma P.A. Lemke 1964 (H)

Stereaceae Pilát

Matula Massee 1888 (H)

Sebacinales M. Weiss et al.

Sebacinaceae K. Wells \& Oberw.

Chaetospermum Sacc. 1892 (C)

Craterocolla Bref. 1888 
Ditangium P. Karst. 1867 (H)

Flahaultiella Seifert 2009 (H)

Opadorhiza T.F. Andersen \& R.T. Moore 1996 (H)

Piriformospora Sav. Verma et al. 1998 (H)

Thelephorales Corner ex Oberw.

Thelephoraceae Chevall.

Parahaplotrichum W.A. Baker \& Partr. 2001 (H)

Agaricomycetes, genera incertae sedis

Akenomyces G. Arnaud ex D. Hornby 1984

Arthrodochium R.F. Castañeda \& W.B. Kendr. 1990 (H)

Arualis Katz 1980 (H)

Bartheletia G. Arnaud ex Scheuer et al. 2008 (H)

Cenangiomyces Dyko \& B. Sutton 1979 (H)

Cruciger R. Kirschner \& Oberw. 1999 (H)

Dendrosporomyces Nawawi et al. 1977

(H)

Ellula Nag Raj 1980 (C)

Fibulocoela Nag Raj 1978 (C)

Fibulotaeniella Marvanová \& Bärl. 1988

(H)

Geotrichopsis Tzean \& Estey 1991 (H)

Gloeosynnema Seifert \& G. Okada 1988 (H)

Glomerulomyces A.I. Romero \& S.E. López 1989 (H)

Glutinoagger Sivan. \& Watling 1980 (H)

Myriococcum Fr. 1823 (H)

Nyctalina G. Arnaud 1952 (H)

Pagidospora Drechsler 1960 (H)

Pycnovellomyces R.F. Castañeda 1987 (C)

Riessia Fresen. 1852 (H)

Riessiella Jülich 1985 (H)

Titaeella G. Arnaud ex K. Ando \& Tubaki 1985 (H)

Tricladiomyces Nawawi 1985 (H)

Class Dacrymycetes Doweld

Dacrymycetales Henn.

Dacrymycetaceae J. Schröt.

Cerinosterus R.T. Moore 1987 (H)

Dacryoscyphus R. Kirschner \& Zhu L. Yang 2005 (H)

Class Tremellomycetes Hibbett et al.
Cystofilobasidiales Fell et al.

Cystofilobasidiaceae K. Wells \& Bandoni

Guehomyces Fell \& Scorzetti 2004 (H)

Itersonilia Derx 1948 (H)

Rhodozyma Phaff et al. 1972 (H)

Mrakiaceae X.Z. Liu et al.

Mrakiella Margesin \& Fell 2008 (H)

Phaffia M.W. Mill. et al. 1976 (Y)

Tausonia Babeva 1998 (H)

Udeniomyces Nakase \& Takem. 1992 (Y)

Filobasidiales Jülich

Filobasidiaceae L.S. Olive

Cryptococcus Vuill. 1901 (Y)

Tremellales Fr.

Cuniculitremaceae J.P. Samp. et al.

Fellomyces Y. Yamada \& I. Banno 1984

(Y)

Kockovaella Nakase et al. 1991 (Y)

Sterigmatosporidium G. Kraep. \& U. Schulze 1983 (Y)

Tremellina Bandoni 1986 (H)

Hyaloriaceae Lindau

Helicomyxa R. Kirschner \& Chee J. Chen 2004 (H)

Tremellaceae Fr.

Anastomyces W.P. Wu et al. 1997 (H)

Bullera Derx 1930 (Yeast)

Cryptococcus Vuill. 1901 (Y)

Hormomyces Bonord. 1851 (H)

Biatoropsis Räsänen 1934 with

hormomyces-like asexual morph $(\mathbf{H})^{\#}$

Tsuchiyaea Y. Yamada et al. 1988 (H)

Trichosporonaceae Nann.

Aegeritella Bałazy \& J. Wiśn. 1974 (H)

Trichosporon Behrend 1890 (Y)

Tritirachium Limber 1940 (H)

Tremellales, genera incertae cedis

(Y)

Derxomyces F.Y. Bai \& Q.M. Wang 2008

(Y)

Hannaella F.Y. Bai \& Q.M. Wang 2008

Moniliella Stolk \& Dakin 1966 (H)

Tremellomycetes, genera incertae sedis 
Heteromycophaga P. Roberts 1997 (Yeastlike)

Moniliella Stolk \& Dakin 1966 (H)

Trichosporonoides Haskins \& J.F.T. Spencer 1967 (H)

AGARICOMYCOTINA, genera incertae sedis Microstella K. Ando \& Tubaki 1984 (H)

Subphylum PUCCINIOMYCOTINA R. Bauer et al.

Class Agaricostilbomycetes R. Bauer et al. Agaricostilbales Oberw. \& R. Bauer

Agaricostilbaceae Oberw. \& R. Bauer Agaricostilbum J. Wright 1970 (H)

Bensingtonia Ingold 1986 (Y)

Sterigmatomyces Fell 1966 (Y)

Chionosphaeraceae Oberw. \& Bandoni

Kurtzmanomyces Y. Yamada et al. 1989 (Y)

Mycogloea L.S.Olive 1950 (Y)

Class Atractiellomycetes R. Bauer et al.

Atractiellales Oberw. \& Bandoni

Phleogenaceae Gäum.

Atractiella Sacc. 1886 (H)

Basidiopycnides J. Reid et al. 2008 (H)

Saccoblastiaceae Jülich

(H)

Infundibura Nag Raj \& W.B. Kendr. 1981

Atractiellales, genera incertae sedis

Atractogloea Oberw. \& Bandoni 1982 (H)

Hobsonia Berk. ex Massee 1891 (H)

Leucogloea R. Kirschner 2004 (H)

Tricladiomyces Nawawi 1985 (H)

Pachnocybales R. Bauer et al.

Pachnocybaceae Oberw. \& R. Bauer

Pachnocybe Berk. 1836 (H)

Class Classiculomycetes R. Bauer et al.

Classiculales R. Bauer et al.

Classiculaceae Bauer et al.

Jaculispora H.J. Huds. \& Ingold 1960 (H)

Naiadella Marvanová \& Bandoni 1987 (H)

Class Cryptomycocolacomycetes R. Bauer et al.

Cryptomycocolacales Oberw. \& R. Bauer
Cryptomycocolacaceae Oberw. \& R. Bauer Colacosiphon R. Kirschner et al. 2001 (H)

Class Cystobasidiomycetes R. Bauer et al. Erythrobasidiales, genera incertae sedis Bannoa Hamam. 2002 (Y)

Erythrobasidium Hamam. et al. 1988 (Y)

Cystobasidiomycetes genera incertae sedis

Cyrenella Goch. 1981 (H)

Class Microbotryomycetes $\mathrm{R}$. Bauer et al. Heterogastridiales Oberw. \& R. Bauer

Heterogastridiaceae Oberw. \& R. Bauer Hyalopycnis Höhn. 1918 (C)

Leucosporidiales J.P. Samp. et al. Leucosporidiaceae Jülich Leucosporidiella Samp. 2003 (Y)

Microbotryales genera incertae sedis Reniforma Pore \& Sorenson 1990 (Y)

Sporidiobolales Doweld

Sporidiobolaceae R.T. Moore

Blastoderma B. Fisch. \& Brebeck 1894

(Y)

Rhodomyces Wettst. 1885 (H)

Sporobolomyces Kluyver \& C.B. Niel 1924 (H)

Sporidiobolales, genera incertae cedis

Ballistosporomyces Nakase et al.

Erythrobasidium Hamam. et al (Y)

Rhodotorula F.C. Harrison 1927 (Y)

Microbotryomycetes, genera incertae sedis

Crucella Marvanová \& Suberkr. 1990 (H)

Class Pucciniomycetes R. Bauer et al.

Helicobasidiales R. Bauer et al.

Helicobasidiaceae P.M. Kirk

Thanatophytum Nees 1816 (H)

Tuberculina Tode ex Sacc. 1880 (H)

Platygloeales R.T. Moore

Eocronartiaceae Jülich

Glomopsis D.M. Hend. 1961 (H)

Platygloeaceae Racib.

Infundibura Nag Raj \& W.B. Kendr. 1981

(H) 
Leucogloea R. Kirschner 2004 (H)

Uncolaceae Buriticá

Calidion Syd. \& P. Syd. 1919 (R)

Pucciniales Clem. \& Shear

Coleosporiaceae Dietel

Chrysomyxa Unger 1840 (R)

Cronartiaceae Dietel

Cronartium Fr. 1815 (R)

Phakopsoraceae Cummins \& Hirats. f. Aeciure Buriticá \& J.F. Hennen 1994 (R)

Macabuna Buriticá \& J.F. Hennen 1994

Malupa Y. Ono et al. 1992 (R)

Milesia F.B. White 1878 (R)

Physopella Arthur 1906 (R)

Uredendo Buriticá \& J.F. Hennen 1994

(R)

Uredostilbe Buriticá \& J.F. Hennen 1994

(R)

0113607426

Phragmidiaceae Corda

Gerwasia Racib. 1909 (R)

Physonema Lév. 1847 (R)

Puccineaceae Chevall.

Caeoma Link 1809 (R)

Roestelia Rebent. 1804 (R)

Pucciniastraceae Gäum. ex Leppik

Milesia F.B. White 1878 (R)

Peridiopsora Kamat \& Sathe 1969 (R)

Pomatomyces Oerst. 1864 (R)

Pucciniastrum G.H. Otth 1861 (R)

Uropyxidaceae Cummins \& Y. Hirats.

Canasta A.A. Carvalho \& J.F. Hennen 2010 (R)

Pucciniales, genera incertae sedis

Elateraecium Thirum. et al. 1966 (R)

Intrapes J.F. Hennen \& Figueiredo 1979

(R)

Uraecium Arthur 1933 (R)

Uredo Pers. 1801 (R)

Septobasidiales Couch ex Donk

Septobasidiaceae Racib.

(H)

Johncouchia S. Hughes \& Cavalc. 1983
Subphylum USTILAGINOMYCOTINA R.

Bauer et al.

Class Exobasidiomycetes Begerow et al.

Doassansiales R. Bauer \& Oberw.

Doassansiaceae R.T. Moore ex P.M. Kirk et al. Savulescuella Cif. 1959 (H)

Entylomatales R. Bauer \& Oberw.

Entylomataceae R. Bauer \& Oberw.

Entylomella Höhn. 1924 (H)

Tilletiopsis Derx 1948 (H)

Exobasidiales Henn.

Brachybasidiaceae Gäum.

Meira Boekhout et al. 2003 (H)

Cryptobasidiaceae Malençon ex Donk

Acaromyces Boekhout et al. 2003 (H)

Exobasidiales genera incertae sedis

Cladostergigma Pat. 1892 (H)

Clinoconidium Pat. 1898 (H)

Coniodictyum Har. \& Pat. 1909 (H)

Golubeviales Q.M. Wang et al.

Golubeviaceae Q.M. Wang et al. 2015

Golubevia Q.M. Wang et al. 2015 (H)*

Microstromatales R. Bauer \& Oberw.

Microstromataceae Jülich

Microstroma Niessl 1861 (H)

Quambalariaceae Z.W. de Beer et al. Quambalaria J.A. Simpson 2000 (H)

Microstromatales genera, incertae sedis Sympodiomycopsis Sugiy. et al. 1991 (Y)

Class Ustilaginomycetes $\mathrm{R}$. Bauer et al.

Urocystidales R. Bauer \& Oberw.

Glomosporiaceae Cif.

Rhombiella Liro 1939 (H)

Thecaphorella H. Scholz \& I. Scholz 1988

(H)

Ustilaginales $\mathrm{G}$. Winter

Anthracoideaceae Denchev

Crotalia Liro 1938 (Y)

Cintractiellaceae Vánky

Naiadella Marvanová \& Bandoni 1987 (H) 
Ustilaginaceae Tul. \& C. Tul.

Pseudozyma Bandoni emend. Boekhout 1985 (H)

Ustilaginomycetes, orders incertae sedis

Urocystidiales R. Bauer \& Oberw.

Urocystidaceae Begerow et al.

Paipalopsis J.G. Kühn 1882 (H)

USTILAGINOMYCOTINA, order incertae sedis

Malasseziales R.T. Moore

Malasseziaceae Denchev \& R.T. Moore

Malassezia Baill. 1889

USTILAGINOMYCOTINA, genera incertae sedis

Botryoconis Syd. \& P. Syd. 1906 (H)

Tilletiella Zambett. 1970 (H)

BASIDIOMYCOTA, class incertae sedis

Wallemiomycetes Zalar et al.

\section{Notes}

In this section we provide taxonomic notes for newly introduced genera since 2012 and notes on genera for which taxonomic placements have been made.

Acanthohelicospora Boonmee \& K.D. Hyde Boonmee et al. (2014) introduced this genus which resided in Tubeufiaceae. Lu et al. (2017c) reported that this genus has hyphomycetous asexual morph.

Acervuloseptoria Crous \& Jol. Roux

Crous et al. (2014c) introduced this genus and phylogenetic analysis showed that it belongs in Mycosphaerellaceae.

\section{Achroiostachys L. Lombard \& Crous}

Lombard et al. (2016) introduced this coelomycetous genus and showed that it belongs in Stachybotryaceae.

Acicuseptoria Quaedvl. et al. Quaedvlieg et al. (2013) introduced this coelomycetous genus and phylogenetic analyses showed that it belongs to Leptosphaeriaceae.

Acidea Hujslová \& M. Kolařík
Wallemiales Zalar et al.

Wallemiaceae R.T. Moore

Wallemia Johan-Olsen 1887 (H)

BASIDIOMYCOTA, genera incertae sedis

Anguillomyces Marvanová \& Bärl. 2000

(H)

Arcispora Marvanová \& Bärl. 1998 (H)

Bartheletica G. Arnaud 2008 (H)

Gloeosynnema Seifert \& G. Okada 1988

(H)

Lactydina Subram. 1978 ('1977') (H)

Microstella K. Ando \& Tubaki 1984 (H)

Nodulospora Marvanová \& Bärl. 2000 (H)

Pseudohelicomyces Garnica \& E. Valenz. $2000(\mathbf{H})$

Trichosporonoides Haskins \& J.F.T. Spencer 1967 (H)

Stachycoremium Seifert 1986 (H)

Stauriella Sivichai \& E.B.G. Jones 2004 (H)

Hujslová et al. (2014) introduced this hyphomycetous genus and showed that it belongs in Helotiales.

Acidomelania E. Walsh \& N. Zhang

Walsh et al. (2014) introduced this hyphomycetous genus and showed that it belongs in Helotiales.

Acidothrix Hujslová \& M. Kolařík

Hujslová et al. (2014) introduced this hyphomycetous genus and showed that it belongs in Amplistromataceae.

Acremoniopsis Giraldo et al.

Crous et al. (2014d) introduced this hyphomycetous genus and showed that it belongs to Hypocreales, genera incertae sedis.

Adautomilanezia Gusmão et al.

Crous et al. (2016a) introduced this hyphomycetous genus and accepted as a genus belongs in Chaetosphaeriaceae.

Aegeanispora E.B.G. Jones \& Abdel-Wahab Abdel-Wahab et al. (2017) introduced this coelomycetous genus and showed that it belongs in Pleosporales genera incertae sedis. 


\section{Aequabiliella Crous}

Crous et al. (2015d) introduced this genus and showed that it belongs to Phaeomoniellales, genera incertae sedis. However, Index Fungorum (2015) introduced Phaeomoniellaceae and placed this genus.

Alanphillipsia Crous \& M.J. Wingf.

Crous et al. (2013b) introduced this coelomycetous genus. Wijayawardene et al. (2014, 2016) showed that Alanphillipsia belongs in Botryosphaeriaceae.

Albifimbria L. Lombard \& Crous

Lombard et al. (2016) introduced this genus and showed that it belongs in Stachybotryaceae.

Alfaria Crous et al.

Wijayawardene et al. (2016) showed that Alfaria has coelomycetous asexual morph based on their phylogenetic analyses. Lombard et al. (2016) also showed that Alfaria has hyphomycetous asexual morphs.

Alfariacladiella Crous \& R.K. Schumach.

Hernández-Restrepo et al. (2016) introduced this hyphomycetous genus and showed that it belongs to Stachybotryaceae.

\section{Allantophomopsiella Crous}

Crous et al. (2014b) introduced this coelomycetous genus and showed that it belongs in Phacidiaceae.

Alloconiothyrium Verkley et al.

Verkley et al. (2014) introduced this genus and phylogenetic analyses showed that it belongs in Didymosphaeriaceae.

Allophaeosphaeria Ariyaw. et al.

Ariyawansa et al. (2015a) introduced $A$. subcylindrospora W.J. Li et al. which showed coelomycetous morphology. Ariyawansa et al. (2015a) also showed that the genus belongs in Phaeosphaeriaceae in their phylogenetic analyses.

Allophoma Q. Chen \& L. Cai
Chen et al. (2015) introduced this genus and showed that it belongs to Didymellaceae in their phylogenetic analyses.

Alternariaster E.G. Simmons

Ariyawansa et al. (2015b) accepted the placement of Alternariaster in Leptosphaeriaceae.

Alveariospora Meir. Silva et al.

Silva et al. (2012) introduced this monotypic, hyphomycetous genus. Sequence data are unavailable thus, taxonomic placement is uncertain.

Ameroconium U. Braun \& Zhurb.

Zhurbenko \& Braun (2013) introduced the lichenicolous hyphomycetous taxon, Ameroconium, with A. cladoniae U. Braun \& Zhurb. as the type species. Sequence data are unavailable; thus, the genus was treated as Ascomycota, genera incertae sedis.

Amphisphaeria Ces. \& De Not.

Liu et al. (2015) introduced A. sorbi Senanayake \& K.D. Hyde with coelomycetous asexual morph.

Amphisphaeriales D. Hawksw. \& O.E. Erikss. Senanayake et al. (2015) reinstated the order but Maharachchikumbura et al. (2016) did not agree with this. However, Hongsanan et al. (2017) accepted Amphisphaeriales.

Anabahusakala Carmo et al. 2014

Carmo et al. (2014) introduced this monotypic, hyphomycetous genus. Sequence data is unavailable, thus its taxonomic placement is uncertain.

Anacoronospora J.S. Monteiro et al.

Monteiro et al. (2016b; taxonomy) introduced this hyphomycetous genus. DNA sequences are unavailable thus placed in Ascomycota genera incertae sedis.

Anacacumisporium Y.R. Ma \& X.G. Zhang $\mathrm{Ma}$ et al. (2016b) introduced this hyphomycetous genus and showed it belongs in Chaetosphaeriaceae in their phylogenetic analyses. 
Anacraspedodidymum C.R. Silva et al. 2014

Silva et al. (2014) introduced this hyphomycetous genus and transferred Craspedodidymum hyalosporum Bhat \& W.B. Kendr. to the new genus. Sequence data are unavailable, thus taxonomic placement is uncertain.

Anaexserticlava T.S. Santa Izabel et al.

Santa-Izabel et al. (2015) introduced this hyphomycetous genus. Sequence data is unavailable, thus taxonomic placement is uncertain.

Anapleurothecium Hern.-Restr. et al.

Hernández-Restrepo et al. (2017) introduced this hyphomycetous genus and showed that it belongs in Pleurotheciaceae, Pleurotheciales.

Anaseptoidium R.F. Castañeda et al.

Castañeda-Ruiz et al. (2012b) introduced this monotypic, hyphomycetous genus, but sequence data is unavailable. Hence, taxonomic position is uncertain.

Angustimassarina Thambug. et al.

Thambugala et al. (2015b) introduced this genus with a hyphomycetous asexual morph. Phylogenetic analyses showed that it belongs to Amorosiaceae.

Annellosympodiella Crous \& Assefa

Crous et al. (2014c) introduced this monotypic, hyphomycetous genus. Wijayawardene et al. (2014) listed Annellosympodiella under Mycosphaerellaceae.

Annulohypoxylon Y.M. Ju et al.

Liu et al. (2015) showed that A. nitens (Ces.) Y.M. Ju \& J.D. Rogers produces nodulosporium-like asexual morphs in the cultures.

Antealophiotrema A. Hashim. \& Kaz. Tanaka Hashimoto et al. (2017) introduced this genus with hyphomycetous asexual morph. Phylogenetic analyses showed that it belongs in Pleosporales.

Anaverticicladus P.O. Costa et al.

Costa et al. (2016) introduced this hyphomycetous genus. DNA sequences are unavailable thus placed in Ascomycota genera incertae sedis

Apenidiella Quaedvl. \& Crous

Quaedvlieg et al. (2014) introduced this monotypic, hyphomycetous genus. Phylogenetic analyses showed that it belongs in Teratosphaeriaceae (Quaedvlieg et al. 2014; Wijayawardene et al. 2014).

Aphanophora Réblová \& Unter.

Réblová et al. (2013) introduced Aphanophora with A. eugeniae (Crous \& Alfenas) Réblová \& Unter. as the type species. Phylogenetic analyses showed that Aphanophora resides in Chaetothyriaceae.

Apiculospora Wijayaw. et al.

Wijayawardene et al. (2016) introduced this monotypic coelomycetous genus and showed that it belongs to Helotiales, genera incertae sedis.

Apiognomonia Höhn.

Senanayake et al. (2017b) illustrated the coelomycetous morph of Apiognomonia veneta, the type species of Apiognomonia. Moreover, Senanayake et al. (2017b) treated Discula Sacc. as a synonym of Apiognomonia.

Apiosporopsis (Traverso) Mariani.

Senanayake et al. (2017b) introduced a new family Apiosporopsidaceae (in Diaporthales) to place this genus.

Apoharknessia Crous \& S.J. Lee

Senanayake et al. (2017b) introduced new family Apoharknessiaceae (in Diaporthales) to place this genus.

\section{Aposphaeria Sacc.}

Tian et al. (2015) accepted this genus as a member in Melanommataceae.

Apseudocercosporella Videira \& Crous

Videira et al. (2016) introduced this hyphomycetous genus and showed it belongs in Mycosphaerellaceae.

Aquanectria L. Lombard \& Crous

Lombard et al. (2015) introduced this genus with a hyphomycetous asexual morph. 
Phylogenetic analyses confirmed its placement in Nectriaceae.

Aquapteridospora J. Yang et al.

Yang et al. (2015) introduced this hyphomycetous genus and showed that it belongs in Diaportheomycetidae.

\section{Aquasubmersa K.D. Hyde \& Huang Zhang}

Zhang et al. (2012a) introduced this coelomycetous genus and showed it belongs in Lophiotremataceae. Ariyawansa et al. (2015a) introduced the holomorph of A. japonica A. Hashim. \& Kaz. Tanaka. Hashimoto et al. (2017) introduced Aquasubmersaceae to place this genus.

Arthrocatena E. Egidi \& L. Selbmann

This hyphomycetous genus was introduced by Egidi et al. (2014), and in sequence data analyses, it grouped in Capnodiales, genera incertae sedis.

\section{Arthrocladium Papendorf}

Nascimento et al. (2016) reinstated this genus with three species. The phylogenetic analyses placed Arthrocladium and Trichomerium as sister clades and confirmed its placement in Trichomeriaceae.

Arthromoniliphora S.S. Silva et al.

Silva et al. (2017) introduced this hyphomycetous genus. DNA sequences are unavailable thus placed in Ascomycota incertae sedis.

\section{Ascochyta Lib.}

Jayasiri et al. (2017b) reported both asexual and sexual morph of Ascochyta medicaginicola var. medicaginicola.

\section{Ascoconidium Seaver}

Johnston et al. (2014) proposed to adopt asexual typified name Ascoconidium over Sageria A. Funk.

Ascocoryne J.W. Groves \& D.E. Wilson Johnston et al. (2014) proposed to adopt sexual typified name Ascocoryne over Coryne Nees, Pirobasidium Höhn. Pleurocolla Petr. and Endostilbum Malençon.

\section{Asteridiella McAlpine}

Hongsanan et al. (2015) reported this genus has an asexual morph which is directly produced from hyphae.

Asterodiscus Voglmayr et al.

Voglmayr et al. (2016) introduced this genus with coelomycetous asexual morph.

\section{Asterodothis Theiss.}

Hongsanan et al. (2014) treated Asterostromina Bat. \& A.F. Vital as synonym of Asterodothis and transferred Asterodothis to Dothideomycetes, genera incertae sedis.

\section{Asterosporium Kunze}

Senanayake et al. (2017b) introduced new family Asterosporiaceae (in Diaporthales) to place this genus.

\section{Atrocalyx A. Hashim. \& Kaz. Tanaka}

Hashimoto et al. (2017) introduced this genus with coelomycetous asexual morph. Phylogenetic analyses showed that it belongs in Lophiotremataceae.

Atrogeniculata J.S. Monteiro et al.

Monteiro et al. (2014b) introduced this hyphomycetous genus. DNA sequences is lacking, thus the genus placed in Ascomycota incertae sedis.

Atrokylindriopsis Y.R. Ma \& X.G. Zhang Ma et al. (2015) introduced this genus to accommodate $A$. setulosa. The sequence was similar to Cladophialophora and Exophiala. The ITS and LSU sequences indicate its placement in Chaetothyriales, genera incertae sedis.

Atrosynnema J.W. Xia et al.

$\mathrm{Xia}$ et al. (2016) introduced this hyphomycetous genus.

Aurantioporthe G. Beier \& R.A. Blanchette Beier et al. (2015) introduced this genus with hyphomycetous asexual morph and phylogenetic analyses showed that it belongs to Cryphonectriaceae. Senanayaka et al. (2017) also accepted this placement.

Aurantiosacculus Dyko \& B. Sutton 
Crous et al. (2012b) shows this genus belongs in Cryphonectriaceae (Diaporthales) in their phylogenetic analyses. Maharachchikumbura et al. (2015) agreed with this placement.

Auratiopycnidiella Crous \& Summerell

Crous et al. (2012b) introduced this monotypic coelomycetous genus and showed it belongs to Diaporthales, genera incertae sedis. Senanayake et al. (2017b) introduced Auratiopycnidiellaceae.

Austropleospora R.G. Shivas \& L. Morin

Ariyawansa et al. (2015a) showed that this genus has coelomycetous asexual morph and confirmed its placement in Didymosphaeriaceae.

\section{Bactrodesmium Cooke}

Tanaka et al. (2015) showed that this genus belongs in Massarineae, Pleosporales.

Bambusicola D.Q. Dai \& K.D. Hyde

Dai et al. (2012) introduced this genus with a coelomycetous asexual morph. Hyde et al. (2013) introduced Bambusicolaceae to accommodate Bambusicola as it shows a distinct lineage in Pleosporales.

Bambusicularia Klaubauf et al.

Klaubauf et al. (2014) introduced this genus and showed that it belongs in Pyriculariaceae, Magnaporthales in their phylogenetic analyses.

\section{Banksiophoma Crous}

Crous et al. (2017) introduced this genus and showed that it belongs in Phaeosphaeriaceae.

Barbatosphaeriaceae $\mathrm{H}$. Zhang et al.

Zhang et al. (2017) introduced this family to accommodate Barbatosphaeria Réblová.

Barrenia E. Walsh \& N. Zhang

Walsh et al. (2015) introduced this hyphomycetous genus and showed that it belongs in Helotiales.

Barretomyces Klaubauf et al.

Klaubauf et al. (2014) introduced this monotypic, hyphomycetous genus and showed that it belongs in Pyriculariaceae, Magnaporthales in their phylogenetic analyses.
Beltraniopsis Bat. \& J.L. Bezerra

Crous et al. (2014c) introduced B. neolitseae Crous \& Summerell and showed that it belongs in Xylariales genera incertae sedis.

Biscogniauxia Kuntze 1891

Liu et al. (2015) showed that B. marginata (Fr.: Fr.) Pouzar produces nodulosporium-like asexual morphs in culture.

Bicoloromyces Heuchert et al.

Heuchert et al. (2015) introduced this lichenicolous, hyphomycetous genus. DNA sequence data is unavailable, thus taxonomic placement is uncertain.

Bisifusarium L. Lombard et al.

Lombard et al. (2015) introduced this genus to accommodate several Fusarium species which are not congeneric with Fusarium sensu stricto. Phylogenetic analyses confirmed its placement in Nectriaceae.

Blastophorum Matsush.

Hyde et al. (2016) introduced B. aquaticum Z.L. Luo et al. and showed that it belongs in Reticulascaceae.

\section{Blumeriella Arx}

Johnston et al. (2014) proposed to adopt sexual typified name Blumeriella over Microgloeum Petr. and Phloeosporella Höhn.

Boeremia Aveskamp et al.

Jayasiri et al. (2017b) introduced Boeremia galiicola Jayasiri et al. with both asexual and sexual morphs.

Botryosphaeria Ces. \& De Not.

The genus Botryosphaeria has Fusicoccum and Dichomera asexual morphs (Phillips et al. 2013, Wijayawardene et al. 2016). Thus, Wijayawardene et al. $(2014,2017)$ proposed to reduce both latter asexual genera under Botryosphaeria.

\section{Botryosporium Corda}

Choi et al. (2014) generated DNA sequences of B. longibrachiatum. The mega blast results showed that this species resides in Sordariomycetes.

Brachycephala J.S. Monteiro et al. 
Monteiro et al. (2015) introduced this hyphomycetous genus but sequence data is unavailable. Hence, taxonomic placement is uncertain.

\section{Brachysporiella Bat.}

Hernández-Restrepo et al. (2017) treated this genus as Kirschsteiniotheliales genera incertae sedis based on B. navarrica

\section{Bradymyces Hubka et al.}

Hubka et al. (2014) introduced this genus and showed that it belongs in Trichomeriaceae.

\section{Braunomyces V.A. Melnik \& Crous}

Mel'nik \& Crous (2014) introduced this monotypic, hyphomycetous genus. Sequence data are unavailable, thus taxonomic placement is uncertain.

Breviappendix Senan. \& K.D. Hyde

Senanayake et al. (2017) introduced this genus with coelomycetous asexual morph and showed it belongs in Sydowiellaceae.

Brevistachys L. Lombard \& Crous

Lombard et al. (2016) introduced this genus and showed that it belongs in Stachybotryaceae.

Briancoppinsia Diederich et al.

Diederich et al. (2012) introduced this new lichenicolous genus by using LSU and SSU. Briancoppinsia belongs in Arthoniaceae.

Brunneiperidium Daranagama et al.

Daranagama et al. (2015) introduced this genus with a hyphomycetous asexual morph. Phylogenetic analyses showed that Brunneiperidium can be accommodated in Xylariaceae.

\section{Brunneodinemasporium Crous \& R.F. Castañeda \\ Crous et al. (2012d) introduced this genus to accommodate dinemasporium-like taxa grouping away from Dinemasporium sensu stricto in Chaetosphaeriaceae, Chaetosphaeriales.}

Bryochiton Döbbeler \& Poelt

Wäli et al. (2014) reported Bryochiton sp. with chlamydospores-like stages (meristematic morph) from cultures. Bryochiton sp. resides in Teratosphaeriaceae in their phylogenetic analyses.

Budhanggurabania $\mathrm{P}$. Wong et al.

Crous et al. (2015d) introduced this genus and phylogenetic analyses showed that it belongs in Magnaporthaceae.

\section{Bullanockia Crous}

Crous et al. (2016a) introduced this hyphomycetous genus and accepted as a genus in Bionectriaceae.

Bussabanomyces Klaubauf et al.

Klaubauf et al. (2014) introduced this monotypic, hyphomycetous genus and showed that it belongs to Magnaporthaceae, Magnaporthales in their phylogenetic analyses.

Caeruleoconidia to Zhurb. \& Pino-Bodas Zhurbenko et al. (2015) introduced Caeruleoconidia Zhurb. \& Diederich but it was not validly published (Index Fungorum 2017). Thus, Caeruleoconidia to Zhurb. \& PinoBodas was introduced to accommodate Caeruleoconidia Zhurb. \& Diederich (Zhurbenko \& Pino-Bodas 2017).

Cainiaceae J.C. Krug

Hongsanan et al. (2017) treated this family as Xylariomycetidae families incertae sedis

\section{Calloria Fr.}

Johnston et al. (2014) proposed to adopt sexual typified name Calloria over Cylindrocolla Bonord.

Calophoma Q. Chen \& L. Cai

Chen et al. (2015) introduced this coelomycetous genus and showed that it belongs to Didymellaceae in their phylogenetic analyses.

Calosphaeria Tul. \& C. Tul.

Réblová et al. (2016a) synonymised Calosphaeriophora Réblová et al. (2004) under Calosphaeria.

Calvolachnella Marinc. et al. 
Hernández-Restrepo et al. (2016) introduced this coelomycetous genus and showed that it belongs in Chaetosphaeriales.

\section{Camarosporium Schulzer}

Tibpromma et al. (2017) reported cucurbitarialike sexual morphs group in Camarosporium sensu stricto.

Camarosporioides W.J. Li \& K.D. Hyde Hyde et al. (2016) introduced this coelomycetous genus and showed that it beongs in Phaeosphaeriaceae.

\section{Camarosporomyces Crous}

Crous \& Groenewald (2017) introduced this coelomycetous genus and Wanasinghe et al. (2017) showed that it be;ongs in Camarosporiaceae.

\section{Capitofimbria L. Lombard \& Crous}

Lombard et al. (2016) introduced this genus and showed that it belongs in Stachybotryaceae.

Camptomeriphila Crous \& M.J. Wingf.

Crous et al. (2016a) introduced this coelomycetous genus and accepted it in Mycosphaerellaceae.

Camptophora Réblová \& Unter.

Réblová et al. (2013) introduced this genus and showed it belongs in Chaetothyriaceae.

\section{Campylospora Ranzoni}

Marvanová \& Laichmanová (2014) introduced Campylospora leptosoma. DNA sequence analyses showed that it belongs to Hypocreomycetidae incertae sedis

\section{Caryophylloseptoria Verkley et al.}

Verkley et al. (2013) introduced Caryophylloseptoria with four coelomycetous species and showed it belongs in Mycosphaerellaceae. Quaedvlieg et al. (2013) accepted this familial placement and added the fifth species.

Castanediella Hern.-Restr. et al.

Crous et al. (2015d) introduced this genus and showed that it belongs in Xylariales, genera incertae sedis. However, Maharachchikumbura et al. (2016) treated it as Sordariomycetes, genera incertae sedis. Hernández-Restrepo et al. (2017) introduced Castanediellaceae.

Catenulomyces Egidi \& de Hoog

This monotypic, hyphomycetous genus was introduced by Egidi et al. (2014). In sequence data analyses, Catenulomyces groups in Capnodiales, genera incertae sedis (Egidi et al. 2014, Wijayawardene et al. 2014).

Cecidiomyces U. Braun \& Zhurb.

Zhurbenko \& Braun (2012) introduced this lichenicolous, hyphomycetous genus. Cecidiomyces lacks sequence data, thus its taxonomic placement is uncertain.

\section{Celerioriella Crous}

Crous et al. (2015d) introduced this genus and showed that it belongs to Phaeomoniellales, genera incertae sedis in their phylogenetic analyses. However, Index Fungorum (2015) introduced Phaeomoniellaceae and placed this genus.

\section{Cephalotrichiella Crous}

Notes: Crous et al. (2014c) introduced this genus and showed it belongs in Microascales, genera incertae sedis in their phylogenetic analyses.

\section{Cercospora Fresen.}

Braun et al. (2013) revised the genus to include mycosphaerella-like sexual morphs.

\section{Chaetoconis Clem.}

Senanayake et al. (2017b) accepted this genu as Diaporthaceae.

Chiangraiomyces Senan. \& K.D. Hyde

Senanayake et al. (2017b) introduced this genus with a coelomycetous asexual morph. In their phylogenetic analyses, they showed that it belongs in Diaporthaceae.

Chlamydotubeufia Boonmee \& K.D. Hyde Brahamanage et al. (2017) introduced $C$. aquatica Brahamanage et al. which represents a hyphomycetous asexual morph.

Chordomyces Bilanenko et al. 
Grum-Grzhimaylo et al. (2016) introduced this hyphomycetous genus and showed it belongs in Plectosphaerellaceae (Glomerellales) in their phylogenetic analyses.

Chrysocrypta Crous \& Summerell

Crous et al. (2012c) introduced this monotypic, coelomycetous genus. Maharachchikumbura et al. (2015) listed Chrysocrypta under Cryphonectriaceae. However, Senanayake et al. (2017b) treated this genus as in Erythrogloeaceae.

Chrysofolia Crous \& M.J. Wingf.

Crous et al. (2015d) introduced this coelomycetous genus and showed that it belongs in Cryphonectriaceae.

\section{Ciliolarina Svrcek}

This genus was reported with septonema-like asexual morphs (Kirk et al. 2008).

Circinoconiopsis A. Hern.-Gut.

Hernández Gutiérrez (2013) introduced this monotypic, hyphomycetous genus. Sequence data are unavailable, thus taxonomic placement is uncertain.

\section{Circinotrichum Nees}

Crous et al. (2015c) showed that this genus belongs in Xylariaceae.

Cistella Quél.

This genus was reported with phialophora-like asexual morphs (Seifert et al. 2011).

Cladosporiopsis S.C. Ren \& X.G. Zhang

Ren et al. (2012) introduced this monotypic, hyphomycetous genus, but sequence data are unavailable. Hence, taxonomic placement is uncertain.

\section{Clathrospora Rabenh.}

This genus was reported with alternaria-like asexual morphs (Kirk et al. 2008).

\section{Claussenomyces Kirschst.}

Johnston et al. (2014) proposed to adopt sexual typified name Claussenomyces over Dendrostilbella Höhn.

Clypeophysalosporaceae Giraldo \& Crous
Giraldo et al. (2017) introduced Clypeophysalosporaceae and accepted three genera as members of it

Coccinonectria L. Lombard \& Crous

Lombard et al. (2015) introduced this genus with a hyphomycetous asexual morph. Coccinonectria resides in Nectriaceae in phylogenetic analyses (Lombard et al. 2015).

Coelodictyosporium Thambug. \& K.D. Hyde Thambugala et al. (2015b) introduced this coelomycetous genus and showed that it belongs in Lophiostomataceae in their phylogenetic analyses.

Collarina Giraldo et al.

Crous et al. (2014d) introduced this hyphomycetous genus and showed that it belongs to Clavicipitaceae.

Collembolispora Marvanová \& Pascoal Crous et al. (2012a) introduced C. aristata and showed that it belongs to Helotiales genera incertae sedis.

\section{Collophorina Damm \& Crous}

The genus Collophora Damm \& Crous was treated as illegitimate (Art. 53.1) thus Wijayawardene et al. (2017) introduced this genus to place species in Collophora.

Conicomyces R.C. Sinclair et al.

Liu et al. (2015) introduced $C$. pseudotransvaalensis and showed that it belongs in Chaetosphaeriaceae.

Conlarium F. Liu \& L. Cai

Liu et al. (2012) introduced this genus with a hyphomycetous asexual morph. Maharachchikumbura et al. (2015) treated Conlarium in Sordariomycetidae, genera incertae sedis. However, Zhang et al. (2017) introduced Conlariaceae (Atractosporales) to place this genus.

\section{Constantinomyces Egidi \& Onofri}

This monotypic, hyphomycetous genus was introduced by Egidi et al. (2014). In sequence data analyses, Constantinomyces groups in Teratosphaeriaceae (Egidi et al. 2014; Wijayawardene et al. 2014). 
Crassicarpon Y. Marín et al.

Marin-Felix et al. (2015) introduced tis genus with hyphomycetous asexual morph. Phylogenetic analyses showed that Crassicarpon belongs in Chaetomiaceae.

Crassimassarina A. Hashim. \& Kaz. Tanaka Hashimoto et al. (2017) introduced this genus with coelomycetous asexual morph. Phylogenetic analyses showed that it belongs in Lophiotremataceae.

Crinitospora B. Sutton \& Alcorn

Crous et al. (2014a) designated an epitype for the type species of this genus and showed it belongs to Melanconidaceae, Diaporthales. However, Senanayake et al. (2017) placed this genus in Stilbosporaceae.

\section{Crucellisporiopsis Nag Raj}

Crous et al. (2014d) introduced C. marquesiae Crous and showed that it belongs to Hyaloscyphaceae, Helotiales.

\section{Crumenulopsis J.W. Groves}

Johnston et al. (2014) proposed to adopt sexual typified name Crumenulopsis over Digitosporium Gremmen.

\section{Cryomyces Selbmann et al.}

This genus was validly published in Wijayawardene et al. (2017)

Cryptoclypeus A. Hashim. \& Kaz. Tanaka Hashimoto et al. (2017) introduced this genus with coelomycetous asexual morph. Phylogenetic analyses showed that it belongs in Lophiotremataceae.

\section{Cryptocoryneum Fuckel}

Hashimoto et al. (2017) introduced Cryptocoryneaceae to place this hyphomycetous genus.

Cryptomeliola S. Hughes \& Piroz.

Hongsanan et al. (2015) reported this genus has asexual morph which is directly produced from hyphae.

Cryptovalsa Ces. \& De Not. ex Fuckel
$\mathrm{Li}$ et al. (2016a) reported that $C$. ampelina (Nitschke) Fuckel has coelomycetous asexual morph.

\section{Cucurbitaria Gray}

This genus was reported with diplodia-like, pyrenochaeta-like asexual morphs (Kirk et al. 2008, Hyde et al. 2013)

\section{Cyclothyriella Jaklitsch \& Voglmayr}

Jaklitsch \& Voglmayr (2016) introduced this genus with coelomycetous asexual morphs and showed that it has distinct lineage in Pleoporales. Hence, Cyclothyriellaceae has been introduced.

Cylindrium Bonord.

Lombard et al. (2015) showed that Cylindrium s. str. resides in Hypocreales genera incertae sedis.

Cylindrocarpostylus R. Kirschner \& Oberw.

Lombard et al. (2015) showed that Cylindrocarpostylus s. str. resides in Nectriaceae in their phylogenetic analyses.

Cylindrodendrum Bonord.

Lombard et al. (2015) and Agustí-Brisach et al. (2016) showed that Cylindrodendrum s. str. resides in Nectriaceae in their phylogenetic analyses.

Cylindrosympodioides Crous \& M.J. Wingf.

Crous et al. (2016b) introduced this hyphomycetous genus and accepted as a genus belongs in Venturiales.

Cymostachys L. Lombard \& Crous

Lombard et al. (2016) introduced this genus and showed that it belongs in Stachybotryaceae.

Cyphellophora G.A. de Vries

Réblová et al. (2013) showed that Cyphellophora has a distinct phylogenetic lineage in Chaetothyriales, thus, they introduced the family Cyphellophoraceae to accommodate Cyphellophora.

Cyphellophoriella Crous \& A. J. Smith

Crous et al. (2015c) introduced this genus and showed that it belongs to Chaetothyriaceae. 
Cytostagonospora Bubák

Quaedvlieg et al. (2013) showed that $C$. martiniana (CBS 135102) belongs in Mycosphaerellaceae.

\section{Cytospora Ehrenb.}

Norphanphoun et al. (2017) revisited the genus and provided comprehensive morphomolecular background of the genus.

Dactylonectria L. Lombard \& Crous

Lombard et al. (2014) introduced this genus with hyphomycetous asexual morph.

Davidsoniella Z.W. de Beer et al.

De Beer et al. (2014) introduced this genus with a hyphomycetous asexual morph. Maharachchikumbura et al. (2015) listed Davidsoniella under Ceratocystidaceae, Microascales.

Dendrophoma Sacc.

Crous et al. (2012d) reinstated the genus which was prviously tread as a synonym of Dinemasporium (Kirk et al. 2008).

Dendryphiella Bubák \& Ranoj.

Boonmee et al. (2016) showed that this hyphomycetous genus belongs in

Dictyosporiaceae

Dendryphion Wallr.

$\mathrm{Su}$ et al. (2016) introduced new species, $D$. aquaticum H.Y. Su \& K.D. Hyde and showed that the genus belongs in Torulaceae.

\section{Dermea Fr.}

Johnston et al. (2014) treated Sphaeronaema Fr. and Foveostroma DiCosmo as synonyms of Dermea

\section{Diatrype Fr.}

Li et al. (2016a) introduced $D$. thailandica R.H. Perera et al. with coelomycetous asexual morph.

Diaporthales Nannf.

Senanayake et al. (2017) revised the order and introduced several new families viz. Apiosporopsidaceae, Apoharknessiaceae,
Asterosporiaceae,

Auratiopycnidiellaceae, Erythrogloeaceae, Melanconiellaceae and Prosopidicolaceae.

Dictyoaquaphila J.S. Monteiro et al.

Monteiro et al. (2016a) introduced this hyphomycetous genus. DNA sequences are unavailable thus placed in Ascomycota genera incertae sedis

Dictyoceratosporella Y.R. Ma \& X.G. Zhang 2016,

$\mathrm{Ma}$ et al. (2016c) introduced this hyphomycetous genus. DNA sequences are unavailable thus placed in Ascomycota genera incertae sedis

Dictyocheirospora D'souza et al.

Boonmee et al. (2016) introduced this hyphomycetous genus and showed that it belongs in Dictyosporiaceae

Dictyopalmispora Pinruan et al.

Boonmee et al. (2016) introduced this hyphomycetous genus and showed that it belongs in Dictyosporiaceae

Dictyospora Brahamanage et al.

Brahamanage et al. (2017) introduced this genus with hyphomycetous asexual morph and showed that it belongs in Tubeufiaceae.

Dictyosporella Abdel-Aziz

Ariyawansa et al. (2015a) introduced this hyphomycetous genus. Zhang et al. (2017) placed it in Diaporthomycetidae, genera incertae sedis.

Dictyosporina Abreu et al.

Hyde et al. (2017) introduced this genus and showed that it belongs in Mycosphaerellaceae.

Dictyosporium Corda

Boonmee et al. (2016) showed that this genus belongs in Dictyosporiaceae

Didymellocamarosporium Wijayaw. \& K.D. Hyde

Wijayawardene et al. (2016) showed that camarosporium-like taxon grouped in Didymellaceae. Hence, Didymellocamaros- 
porium was introduced to accommodate this taxon.

Didysimulans Tibpromma et al.

Tibpromma et al. (2017) introduced this genus and reported chlamydospores from the cultures.

Digicatenosporium S.M. Leão et al.

Leão-Ferreira et al. (2015) introduced this hyphomycetous genus. DNA sequence data are unavailable thus taxonomic placement is uncertain.

\section{Digitatispora Doguet}

Rämä et al. (2014) showed that this genus has phylogenetic lineage in Atheliaceae, Atheliales.

\section{Digitodesmium P.M. Kirk}

Boonmee et al. (2016) showed that this genus belongs in Dictyosporiaceae in their phylogenetic analyses.

\section{Dimorphiseta L. Lombard \& Crous}

Lombard et al. (2016) introduced this genus and showedv that it belongs in Stachybotryaceae.

\section{Dimorphiopsis Crous}

Crous et al. (2013b) introduced Dimorphiopsis with $D$. brachystegiae as the type species. They also queried whether this odd fungus is a coelomycete or hyphomycete. Mega blast results of LSU sequence data show it has close relationship with Lophiostoma sp. (Crous et al. 2013b) and Wijayawardene et al. (2016) confirmed its placement in Lophiostomataceae, Pleosporales.

\section{Diplocarpon F.A. Wolf}

Johnston et al. (2014) proposed to adopt sexual typified name Diplocarpon over Entomosporium Lév., Bostrichonema Ces., Marssonina Magnus, and Entomopeziza Kleb. The genus might show coelomycetous or hyphomycetous asexual morphs.

Diplolaeviopsis Giralt \& D. Hawksw. Pärtel et al. (2016) reinstated the family Cordieritidaceae and showed that Diplolaeviopsis resides in Cordieritidaceae.

Discopycnothyrium Hongsanan \& K.D. Hyde
Hongsanan et al. (2016) introduced this coelomycetous genus and showed that it belongs in Asterinaceae in their phylogenetic analyses.

Disculoides Crous et al.

Crous et al. (2012b) introduced this genus and showed that it belongs in Diaporthales. Maharachchikumbura et al. (2015) also treated Disculoides in Diaporthales, genera incertae sedis. However, Senanayake et al. (2017b) treated this genus as Erythrogloeaceae.

Distophragmia R.F. Castañeda et al.

Castañeda-Ruiz et al. (2015) introduced this hyphomycetous genus. DNA sequence data are unavailable thus taxonomic placement is uncertain.

Distoseptispora K.D. Hyde et al.

Su et al. (2016) introduced this hyphomycetous genus and showed that it belongs to Distoseptisporaceae.

Diversimorbus S.F. Chen \& Jol. Roux Chen et al. (2013) introduced this genus with a coelomycetous sexual morph. Phylogenetic analyses showed that it belongs in Cryphonectriaceae, Diaporthales.

Dokmaia I. Promputtha

Luo et al. (2014) deposited the sequences of Dokmaia monthadangi, the type species of the genus. The megablast results showed that it belongs to Pleosporineae genera incertae sedis.

Ellisembiopsis T.S. Santa Izabel \& Gusmão

Santa-Izabel et al. (2013) introduced this monotypic genus. Sequence data are unavailable; thus the taxonomic placement is uncertain and it is presently placed in Ascomycota, genera incertae sedis.

Emarcea Duong et al.

Crous et al. (2015d) introduced $E$. eucalyptigena Crous \& M.J. Wingf. which resides in Xylariaceae.

Embryonispora G.Z. Zhao

Zhao et al. (2013) introduced this hyphomycetous genus. Sequence data are 
unavailable, thus taxonomic placement is uncertain.

Emericellopsis J.F.H. Beyma

Hyde et al. (2016) introduced E. persica Papizadeh et al. with a hyphomycetous asexual morph.

\section{Endocoryneum Petr.}

Wijayawardene et al. (2016) introduced new species and showed that it belongs to Didymellaceae.

Endomeliola S. Hughes \& Piroz.

Hongsanan et al. (2015) reported this genus has asexual morph which is directly produced from hyphae.

\section{Epicoleosporium Videira \& Crous}

Videira et al. (2016) introduced this hyphomycetous genus. Phylogenetic analyses showed that it belongs in Mycosphaerellaceae.

\section{Epinephroma Zhurb.}

Zhurbenko et al. (2012) introduced this monotypic, lichenicolous, coelomycetous genus. Sequence data is unavailable, hence, taxonomic placement is uncertain.

\section{Eriosporella Höhn.}

Dai et al. (2014c) introduced a new coelomycetous species, E. bambusicola D.Q. Dai et al. and showed it belongs to Capnodiales, genera incertae sedis in their phylogenetic analyses.

\section{Erythrogloeum Petr.}

Crous et al. (2012b) showed that this genus belongs to Diaporthales and designated an epitype for Erythrogloeum hymenaeae, the type species of the genus Erythrogloeum. Senanayake et al. (2017b) introduced Erythrogloeaceae to accommodate the genus.

Escovopsioides H.C. Evans \& J.O. Augustin. Augustin et al. (2012) introduced this genus with hyphomycetous asexual morph. Phylogenetic analyses showed that it belongs in Hypocreaceae.

Etayoa Diederich \& Ertz
Ertz et al. (2014) introduced this genus with a hyphomycetous asexual morph. In phylogenetic analyses, Etayoa resides in Phaeococcomycetaceae, Lichenostigmatales.

Euantennariaceae Hughes \& Corlett

Liu et al. (2017) placed this family in Capnodiales.

Eucalyptostroma Crous \& M.J. Wingf.

Crous et al. (2016a) introduced this coelomycetous genus and accepted it as a genus in Chaetosphaeriaceae.

\section{Eupelte Syd.}

This genus was reported with clasterosporiumlike and septoidium-like asexual morphs (Kirk et al. 2008, Seifert et al. 2011).

Eupenidiella Quaedvl. \& Crous

Quaedvlieg et al. (2014) introduced this monotypic, hyphomycetous genus and showed it belongs in Teratosphaeriaceae in their phylogenetic analyses.

\section{Eutiarosporella Crous}

Crous et al. (2015b) introduced this genus and showed it belongs to Botryosphaeriaceae. Li et al. (2016a) introduced E. dactylidis (Thambug. et al.) Dissanayake et al. which represents a sexual morph of Eutiarosporella.

Exosporodiella Ganie et al.

Ganie et al. (2012) introduced this monotypic coelomycetous genus. Sequence data are unavailable, thus taxonomic placement is uncertain.

Extremus Quaedvl. \& Crous

Quaedvlieg et al. (2014) introduced this hyphomycetous rock inhabiting genus and showed it belong in Teratosphaeriaceae in their phylogenetic analyses.

Falciphora J. Luo \& N. Zhang

Luo et al. (2015) introduced this genus and showed it belongs to Magnaporthaceae in their phylogenetic analyses.

Falciphoriella M. Hern.-Restr. \& Crous 
Hernandez-Restrepo et al. (2016) introduced this hyphomycetous genus and showed it belongs in Magnaporthaceae.

Falcocladium S.F. Silveira et al.

Jones et al. (2014) introduced Falcocladiaceae to place Falcodium in Hypocreomycetidae, order incertae sedis.

Fantasmomyces DongHyeon Lee et al.

Crous et al. $\left(2016^{\mathrm{a}}\right)$ introduced this genus with hyphomycetous asexual morph and accepted as a genus belongs in Sordariomycetes genera incertae sedis.

\section{Filiella Videira \& Crous}

Videira et al. (2016) introduced this hyphomycetous genus. Phylogenetic analyses showed that it belongs in Mycosphaerellaceae.

\section{Filosporella Nawawi}

Baschien et al. (2013) showed that Filosporella s. lato. resides in Helotiales. However, the type species lacks sequence data while ex-type cultures of three species ( $F$. fistucella, $F$. versimorpha and $F$. exilis) reside in Helotiaceae.

\section{Flammocladiella Crous et al.}

Crous et al. (2015c) introduced this genus and showed that it has distinct phylogenetic lineage in Hypocreales. Hence Crous et al. (2015c) introduced Flammocladiaceae to accommodate Flammocladiella. However, Hongsanan et al. (2017) treated Flammocladiaceae as a synonym of Clavicipitaceae.

Fodinomyces Vázquez-Campos \& Neilan Vázquez-Campos et al. (2014) introduced this hyphomycetous genus and showed it belongs in Teratosphaeriaceae.

\section{Foliophoma Crous}

Crous \& Groenewald (2017) introduced this coelomycetous genus. Wanasinghe et al. (2017) showed that it belongs in Pleosporales.

Forliomyces Phukhams. et al.

Phukhamsakda et al. (2016a) introduced this coelomycetous genus and showed that it belongs to Sporormiaceae. Wijayawardene et al. (2016) confirmed this placement in their phylogenetic analyses.

Fumiglobus D.R. Reynolds \& G.S. Gilbert Bose et al. (2014) introduced a new species, $F$. pieridicola and showed Fumiglobus belongs in Capnodiaceae.

Fusiconidium J.F. Li et al.

Li et al. (2017a) introduced this genus which accommodated in Melanommataceae

Fuscosclera Hern.-Restr. et al.

Hernández-Restrepo et al. (2017) introduced this hyphomycetous genus and showed that it belongs in Mollisiaceae, Helotiales.

Fuscosporella J. Yang et al.

Yang et al. (2016) introduced this hyphomycetous genus and showed that it has distinct lineage in Hypocreomycetidae, Sordariomycetes. Thus, Fuscosporellales and Fuscosporellaceae were introduced. However, Hongsanan et al. (2017) showed that Fuscosporellales resided in Savoryellomycetidae.

Fuscostagonospora Kaz. Tanaka \& K. Hiray. Tanaka et al. (2015) introduced this genus and showed that it belongs to Massarineae. Wijayawardene et al. (2016) accepted this placement. However, Hyde et al. (2017) introduced Fuscostagonosporaceae to place this genus.

Fusicolla Bonord.

Lombard et al. (2015) showed that Fusicolla s. str. resides in Nectriaceae in their phylogenetic analyses.

Fusoidiella Videira \& Crous

Videira et al. (2016) introduced this hyphomycetous genus. Phylogenetic analyses showed that it belongs in Mycosphaerellaceae.

Gaeumannomycella M. Hern.-Restr. \& Crous Hernandez-Restrepo et al. (2016) introduced this hyphomycetous genus and showed it belongs in Magnaporthaceae.

Gaeumannomyces Arx \& D.L. Olivier 
Luo et al. (2015) showed that Gaeumannomyces and Harpophora W. Gams grouped as a monophyletic clade in their phylogenetic analyses. Thus, they proposed to adopt Gaeumannomyces over Harpophora.

\section{Galeaticarpa A. Hashim. \& Kaz. Tanaka}

Hashimoto et al. (2017) introduced this genus with coelomycetous asexual morph. Phylogenetic analyses showed that it belongs in Lophiotremataceae.

Geastrumia Bat.

Ismail et al. (2016) showed that this coelomycetous genus belongs in Mycosphaerellaceae in their phylogenetic analyses.

Gelatinomyces N. Sanoamuang et al.

Sanoamuang et al. (2013) introduced this genus with phialophora-like hyphomycetous asexual morph. In phylogenetic analyses, Gelatinomyces grouped in Leotiomycetes, but did not cluster in any family.

Gelatinipulvinella Hosoya \& Y. Otani

Johnston et al. (2014) proposed to adopt sexual typified name Gelatinipulvinella over Aureohyphozyma Hosoya \& Y. Otani.

Globobotrys L. Lombard \& Crous

Lombard et al. (2016) introduced this genus and showed that it belongs in Stachybotryaceae.

Godroniopsis Diehl \& E.K. Cash

Johnston et al. (2014) proposed to adopt sexual typified name Godronia over Sphaeronaema and Dichaenopsella.

Golubevia Q.M. Wang et al.

Wang et al. (2015) introduced this genus which resides in Golubeviales, Golubeviaceae, Exobasidiomycetes.

Grandibotrys L. Lombard \& Crous

Lombard et al. (2016) introduced this genus and showed that it belongs in Stachybotryaceae.

Greeneria Scribn. \& Viala
Senanayake et al. (2017b) accepted the genus as a member in Melanconiellaceae.

Gregatothecium L. Lombard \& Crous

Lombard et al. (2016) introduced this genus and showed that it belongs in Stachybotryaceae.

Hawksworthiomyces Z.W. de Beer et al.

Da Beer et al. (2016) introduced this genus and showed in belongs in Ophiostomatales.

Helicangiospora Boonmee et al.

Boonmee et al. (2014) introduced this genus with a hyphomycetous asexual morph. Phylogenetic analyses showed that it belongs in Tubeufiaceae.

Helicocentralis Sri-indr. Et al.

Sri-indrasutdhi et al. (2015) introduced this hyphomycetous genus and showed that it belongs to Leotiomycetes in their phylogenetic analyses.

Helicodochium J.S. Monteiro et al.

Monteiro et al. (2014a) introduced this monotypic, hyphomycetous genus. Sequence data are unavailable, thus taxonomic placement is uncertain.

Helicosporium Nees Brahamanage et al. (2017) introduced $H$. flavum Brahamanage et al. with hyphomycetous asexual morph.

Helminthosporiella Hern.-Restr. et al.

Crous et al. (2016b) introduced this hyphomycetous genus and accepted it belongs in Massarinaceae.

Helminthosporium Link

Voglmayr \& Jaklitsch (2017) treated Exosporium Link as a synonym of Helminthosporium Link. Moreover, Voglmayr \& Jaklitsch (2017) accepted Helminthosporium has massarina- or splanchnonema-like sexual morphs.

Hermatomyces Speg.

Doilom et al. (2016) introduced several new species and showed that they belong in 
Lophiotremataceae. However, Hashimoto et al. (2017) introduced Hermatomycetaceae to place this genus.

Heterophoma Q. Chen \& L. Cai

Chen et al. (2015) introduced this coelomycetous genus and phylogenetic analyses showed that it belongs in Didymellaceae.

\section{Heterosporicola Crous}

This genus was introduced to place Heterospora (Boerema et al.) Gruyter et al. which was invalidly published in de Gruyter et al. (2012)

Huntiella Z.W. de Beer et al.

De Beer et al. (2014) introduced this genus with a hyphomycetous asexual morph. Phylogenetic analyses showed that it belongs in Ceratocystidaceae.

Hyaliappendispora Senan. et al.

Senanayake et al. (2017b) introduced this genus with coelomycetous asexual morph. In their phylogenetic analyses, they showed that it belongs in Diaporthaceae

\section{Hyalopeziza Fuckel}

This genus was reported with phialophora-like asexual morphs (Kirk et al. 2008).

\section{Hyaloscypha Boud.}

Han et al. (2014) reported that $H$. albohyalina var. spiralis and $H$. albohyalina var. monodictys produce Pseudaegerita and monodictys-like asexual morphs, respectively.

Hyphoconis Egidi \& Quaedvl.

Egidi et al. (2014) introduced this hyphomycetous genus and showed that it belongs in Capnodiales, genera incertae sedis.

Hypsostroma Huhndorf This genus was reported with pleurophomopsis-like asexual morphs (Kirk et al. 2008).

\section{Hypoxylon Bull.}

Li et al. (2016) introduced Camporesia W.J. Li \& K.D. Hyde, a coelomycetous genus and showed that it belongs in Xylariaceae. Based on mega blast searches, Camporesia is treated as a synonym of Hypoxylon (personal communication with M. Stadler)

Hyweljonesia R.G. Shivas et al.

Crous et al. (2016a) introduced this hyphomycetous genus and showed it belongs in Teratosphaeriaceae.

Idriellopsis Hern.-Restr. \& Crous

Hernández-Restrepo et al. (2015) introduced this hyphomycetous genus and molecular phylogenetic analyses showed that it belongs in Sordariomycetes, genera incertae sedis. Maharachchikumbura et al. (2016) accepted as a genus in Xylariales.

Immotthia M.E. Barr

Hyde et al. (2017) illustrated holomorph of the genus.

Inaequalispora L. Lombard \& Crous

Lombard et al. (2016) introduced this genus and showed that it belongs in Stachybotryaceae.

Incertomyces E. Egidi \& L.Z.G. Fonseca Egidi et al. (2014) introduced this rockinhabiting hyphomycetous genus which has poor morphological features. Phylogenetic analyses showed that it belongs in Teratosphaeriaceae.

Irenopsis F. Stevens

Hongsanan et al. (2015) reported this genus has asexual morph which is directly produced from hyphae.

Isthmoconidium Etayo \& Fr. Berger

Etayo \& Berger (2013) introduced this monotypic, hyphomycetous genus. Sequence data are unavailable and thus taxonomic placement it uncertain and it is presently accommodated in Ascomycota, genera incertae sedis.

Jackrogersella L. Wendt et al.

Wendt et al. (2017) introduced this genus with hyphomycetous asexual morph.

Jalapriya D'souza et al. 
Boonmee et al. (2016) introduced this hyphomycetous genus and showed that it belongs in Dictyosporiaceae.

Janetia M.B. Ellis

Da Silva et al. (2016) showed that this coelomycetous genus belongs in Mycosphaerellaceae.

Jayarambhatia J. Pratibha

Pratibha (2013) introduced this monotypic genus. Sequence data are unavailable thus, taxonomic placement is uncertain. It is presently accommodated in Ascomycota, genera incertae sedis.

Johnalcornia Y.P. Tan \& R.G. Shivas

Tan et al. (2014) introduced this genus to accommodate Bipolaris aberrans Alcorn which is distinct from Bipolaris sensu stricto, and is placed in Pleosporaceae.

Juglanconis Voglmayr \& Jaklitsch

Voglmayr et al. (2017) introduced this genus with coelomycetous asexual morph. In the phylogenetic analyses, Juglanconis was placed as a distinct clade in Diaporthales thus Juglanconidaceae was introduced.

\section{Kalmusia Niessl}

The genus Dendrothyrium was introduced by Verkley et al. (2014) with D. variisporum Verkley et al. and phylogenetically clusters in Didymosphaeriaceae (Ariyawansa et al. 2014, Verkley et al. 2014). However, Ariyawansa et al. (2014) showed that the type species of Dendrothyrium, $D$. variisporum and $D$. longisporum Verkley et al. grouped in Kalmusia sensu stricto, thus the former name was reduced to synonymy under the latter generic name.

Kastanostachys L. Lombard \& Crous

Lombard et al. (2016) introduced this genus with hyphomycetous asexual morph and showed that it belongs in Stachybotryaceae.

Kirschsteiniothelia D. Hawksw.

Li et al. (2016a) introduced K. tectonae Doilom et al. with a hyphomycetous asexual morph.
Kirschsteiniotheliaceae S. Boonmee \& K.D. Hyde

$\mathrm{Su}$ et al. (2016) revisited family Kirschsteiniotheliaceae in their phylogenetic analyses.

Kirstenboschia Quaedvl. Et al.

Quaedvlieg et al. (2013) introduced this monotypic, coelomycetous genus. It does not group with any taxa in phylogenetic analyses, thus it was accommodated in Ascomycota, genera incertae sedis. The megablast results indicated this genus shows phylogenetic lineages with genera in Xylariales.

Kohlmeyeriopsis Klaubauf et al.

Klaubauf et al. (2014) introduced this genus with trichocladium-like asexual morphs. Phylogenetic analyses showed that it belongs to Magnaporthaceae.

Koorchalomella Chona et al.

Hyde et al. (2017) introduced K. salmonispora Abdel-Aziz and Abdel-Wahab and showed that it resides in Stachybotryaceae.

Lamproconium (Grove) Grove

Norphanphoun et al. (2016) showed that Lamproconium has distinct phylogenetic lineage in Diaporthales thus introduced Lamproconiaceae.

Lapidomyces de Hoog \& Stielow

Egidi et al. (2014) introduced this hyphomycetous rock-inhabiting genus which has few distinct morphological features. Phylogenetic analyses showed that it belongs in Teratosphaeriaceae (Quaedvlieg et al. 2014).

Lareunionomyces Crous \& M.J. Wingf.

Crous et al. (2016b) introduced this hyphomycetous genus and accepted it belongs in Helotiales.

\section{Latorua Crous}

Crous et al. (2015a) introduced this genus and showed that it has a distinct phylogenetic lineage in Pleosporales. Hence, they introduced Latoruaceae to accommodate the new genus.

Lectera P.F. Cannon 
Cannon et al. (2012) introduced this monotypic coelomycetous genus and phylogenetic analyses showed that it belongs in Plectosphaerellaceae, Hypocreomycetidae, Sordariomycetes.

Lentithecium K.D. Hyde et al.

Hyde et al. (2016) introduced L. unicellulare Abdel-Aziz, a coelomycetous taxon which resides in Lentitheciaceae.

Lentomitella Höhn.

Zhang et al. (2017) introduced Lentomitellaceae to accommodate this genus and accepted the genus with hyphomycetous asexual morphs.

Leptosphaeria Ces. \& De Not.

Hyde et al. (2016) introduced L. cirsii Jayasiri et al. with a coelomycetous asexual morph.

Leptotrochila P. Karst.

Johnston et al. (2014) proposed to adopt sexual typified name Leptotrochila over Sporonema Desm.

Liberomyces Pažoutová et al.

Pažoutová et al. (2012) introduced new endophytic coelomycete genus with two species. Phylogenetic analyses showed it belongs to Sordariomycetes, genera, incertae sedis.

Libertasomyces Crous \& Roets

Crous et al. (2016b) introduced this hyphomycetous (with subverticillium-like synasexual morph), genus and accepted it as a genus in Pleosporales genera incertae sedis. Crous \& Groenewald (2017) introduced Libertasomycetaceae to place this genus.

Licopolia Sacc. et al.

Hyde et al. (2017) illustrated the holomorph of the genus with coelomycetous asexual morph and placed it in Vizellaceae.

Lignosphaeria Boonmee et al.

Thambugala et al. (2015b) introduced this genus with a coelomycetous asexual morph. Phylogenetic analyses showed that it belongs in Dothideomycetes genera incertae sedis.

Lithohypha Selbmann \& Isola
Wijayawardene et al. (2017) introduced this genus to accommodate Lithophila Selbmann \& Isola which was invalidly published in Isola et al. (2015).

Longipedicellata $\mathrm{H}$. Zhang et al.

Zhang et al. (2016) introduced this genus with hyphomycetous asexual morph. Phukhamsakda et al. (2016b) introduced new family, Longipedicellataceae.

Longitudinalis Tibpromma \& K.D. Hyde

Hyde et al. (2017) introduced this hyphomycetous genus and showed that it belongs in Plectosphaerellaceae.

Lophiostoma Ces. \& De Not.

Ariyawansa et al. (2015a) reported a coelomycetous asexual morph in culture of $L$. caulium (Fr.) Ces. \& De Not.

Lunatiannulus Daranagama et al.

Daranagama et al. (2015) this genus with libertella-like asexual morph. Phylogenetic analyses showed that it belongs in Xylariaceae.

Luteocirrhus C.F. Crane et al.

Crane \& Burgess (2013) introduced this coelomycetous genus and showed that it belongs in Cryphonectriaceae.

Macgarvieomyces Klaubauf et al.

Klaubauf et al. (2014) introduced this monotypic, hyphomycetous genus. Phylogenetic analyses showed that it belongs in Pyriculariaceae.

\section{Macrodiplodiopsis Petr.}

Crous et al. (2015a) introduced Macrodiplodiopsidaceae to place Macrodiplodiopsis. Crous et al. (2015a) introduced the sexual morph of $M$. desmazieri (Mont.) Petr., the type species of Macrodiplodiopsis.

Macrohilaceae Crous

Crous et al. (2015a) introduced Macrohilaceae to place Macrohilum.

Magnaporthiopsis J. Luo \& N. Zhang

Luo \& Zhuang (2012) introduced Magnaporthiopsis a phialophora-like asexual genus. Phylogenetic analyses based on SSU, 
ITS, LSU, MCM7, RPB1 and TEF1 genes, showed that it belongs in Magnaporthaceae. Maharachchikumbura et al. (2015) agreed with this familial placement.

Magnibotryascoma Thambug. \& K.D. Hyde Thambugala et al. (2015) introduced this genus and showed that it belongs in Floricolaceae (current name: Teichosporaceae)

Magnicamarosporium Kaz. Tanaka \& K. Hiray.

Tanaka et al. (2015) introduced this coelomycetous genus and showed that it belongs in Sulcatisporaceae in their phylogenetic analyses.

Magnohelicospora R.F. Castañeda et al.

Castañeda et al. (2013) introduced this hyphomycetous genus. Sequences are unavailable thus taxonomic placement was uncertain. Hernández-Restrepo et al. (2017) showed that the genus in Venturiaceae.

Malaysiasca Crous \& M.J. Wingf.

Crous et al. (2016b) introduced this genus with hyphomycetous asexual morph and accepted it as a genus in Glomerellales.

\section{Marasasiomyces Crous}

Crous et al. (2015b) introduced this genus and showed it belongs in Botryosphaeriaceae.

\section{Mastigosporium Riess}

Crous et al. (2014a) showed that this genus belongs to Helotiales genera incertae sedis in their phylogenetic analyses.

Matsushimamyces Rahul Sharma \& Rohit Sharma

Sharma et al. (2015) introduced this coelomycetous genus and phylogenetic analyses showed that it belongs to Latoruaceae (Pleosporales).

Medicopsis Gruyter et al.

Pyrenochaeta romeroi clustered in Trematosphaeriaceae while Pyrenochaeta sensu stricto resides in Cucurbitariaceae. Hence, de Gruyter et al. (2012) introduced Medicopsis to accommodate Pyrenochaeta romeroi.

\section{Megacapitula J.L. Chen \& Tzean}

Prabhugaonkar \& Bhat (2011) showed that this genus resides in Pleosporales genera incertae sedis in their phylogenetic analyses.

Melanocamarosporium Wijayaw. et al.

Wijayawardene et al. (2016) introduced this genus to accommodate camarosporium-like taxon resides in Melanommataceae.

\section{Melanconiella Sacc.}

Senanayake et al. (2017b) accepted the genus with coelomycetous asexual morphs and introduced Melanconiellaceae.

Melanoctona Qing Tian et al.

Tian et al. (2016) introduced this genus with hyphomycetous asexual morph and showed it belongs in Herpotrichiellaceae in their phylogenetic analyses

Melanomma Nitschke ex Fuckel

Hyde et al. (2013) and Tian et al. (2015) reported coelomycetous asexual morphs from this genus.

\section{Melanotrigonum Réblová}

Réblová et al. (2016b) introduced this genus with a hyphomycetous asexual morph. Molecular phylogenetic analyses showed that Melanotrigonum belongs in Pleurotheciaceae.

\section{Meliola Fr.}

Hongsanan et al. (2015) reported this genus has asexual morph which is directly produced from hyphae.

Melnikia Wijayaw. Et al.

Wijayawardene et al. (2016) introduced this coelomycetous genus and showed that it belongs to Phaeosphaeriaceae.

Melnikomyces Crous \& U. Braun

Crous et al. (2014c) introduced this hyphomycetous genus and showed that it belongs in Chaetothyriales, genera incertae sedis.

Menisporopsis S. Hughes

Seifert et al. (2011) accepted this genus as a member in Chaetosphaeriaceae. Réblová et al. 
(2016a) reduced Menisporopascus Matsush. (sexual morph fide Matsushima 2001) under Menisporopsis.

\section{Meristemomyces Isola \& Onofri}

Egidi et al. (2014) introduced this hyphomycetous genus. Phylogenetic analyses showed that it belongs in Teratosphaeriaceae (Quaedvlieg et al. 2014, Wijayawardene et al. 2014).

Metacordyceps G.H. Sung et al.

Wen et al. (2017) introduced $M$. neogunnii T.C. Wen \& K.D. Hyde with hyphomycetous asexual morph.

Metapochonia Kepler et al.

Kepler et al. (2014) introduced this genus which has verticillium-like conidia. Phylogenetic analyses showed that Metapochonia belongs in Clavicipitaceae, Hypocreales.

Microdochiella Hern.-Restr. \& Crous Hernández-Restrepo et al. (2015) introduced this hyphomycetous genus and molecular phylogenetic analyses showed that it belongs in Orbiliales, genera incertae sedis.

\section{Microdochium Syd.}

Réblová et al. (2016a) accepted Microdochium over Monographella Petr.

\section{Microsphaeropsis Höhn.}

Chen et al. (2015) introduced Microsphaeropsidaceae to accommodate Microsphaeropsis since Microsphaeropsis showed distinct phylogenetic lineage from Didymellaceae.

\section{Minimelanolocus R.F. Castañeda \& Heredia} Liu et al. (2015) introduced four new species in this genus and provided the molecular dataset for Minimelanolocus. The phylogenetic analyses showed that Minimelanolocus belongs in Herpotrichiellaceae.

Minteriella Heredia et al.

Heredia et al. (2013) introduced this hyphomycetous genus from Mexico. DNA sequence data unavailable thus placed in Ascomycota genera incertae sedis.
Minutiella Crous

Crous et al. (2015d) introduced this genus and showed that it belongs in Phaeomoniellales, genera incertae sedis. However, Index Fungorum (2015) introduced Phaeomoniellaceae and placed this genus.

Moelleriella Bres.

Li et al. (2016a) introduced M. phukhiaoensis Mongkol. et al. with coelomycetous asexual morph.

Monochaetia (Sacc.) Allesch.

De Silva et al. (2017) introduced new species, M. ilexae N.I. de Silva et al. and confirmed the placement of the genus in Pestalotiopsidaceae (current name: Sporocadaceae)

Monticola Selbmann \& Egidi

Egidi et al. (2014) introduced this hyphomycetous genus. Phylogenetic analyses showed that it belongs in Teratosphaeriaceae (Quaedvlieg et al. 2014, Wijayawardene et al. 2014).

Mucispora J. Yang et al.

Yang et al. (2016) introduced this hyphomycetous genus and showed that belongs in Fuscosporellaceae.

Mucoharknessia Crous et al.

Crous et al. (2015b) introduced this genus and showed it belongs in Botryosphaeriaceae in their phylogenetic analyses. This placement was confirmed by Wijayawardene et al. (2016).

Mulderomyces Crous et al.

Crous et al. (2016b) introduced this coelomycetous genus and accepted it as a genus in Ostropales.

\section{Munkovalsaria Aptroot}

This genus was reported with lecythophora-like asexual morphs (Kirk et al. 2008).

Murilentithecium Wanas. et al.

Wanasinghe et al. (2014) introduced this genus with camarosporium-like asexual morph and showed that this genus resides in Lentitheciaceae. 
Muriphaeosphaeria Phukhams. et al.

Phukhamsakda et al. (2015) introduced this genus with a coelomycetous asexual morph. Phylogenetic analyses confirmed its placement in Phaeosphaeriaceae.

Muripulchra Z.L. Luo et al.

Luo et al. (2017) introduced this aquatic hyphomycetous genus and showed that it belongs in Tubeufiaceae.

Murispora Y. Zhang bis et al.

Wanasinghe et al. (2015) reported phoma-like asexual morphs from the putative culture of $M$. hawksworthii.

\section{Mycocalicium Vain.}

Ariyawansa et al. (2015a) introduced $M$. hyaloparvicellulum Daranagama \& K.D. Hyde with a hyphomycetous asexual morph.

Mycofalcella Marvanová et al.

Baschien et al. (2013) showed that this genus belongs in Helotiaceae.

Mycophilomyces Crous \& M.J. Wingf.

Crous et al. (2016 $\left.{ }^{\mathbf{a}}\right)$ introduced this hyphomycetous genus and accepted as a genus belongs in Clavicipitaceae.

\section{Mycosphaerelloides Videira \& Crous}

Videira et al. (2016) introduced this genus with hyphomycetous asexual morph. In phylogenetic analyses, they showed that it belongs in Mycosphaerellaceae.

\section{Myrtacremonium Crous}

Crous et al. (2017) introduced this coelomycetous genus and showed that it belongs in Niessliaceae, Hypocreales.

\section{Myrtapenidiella Quaedvl. \& Crous}

Quaedvlieg et al. (2014) introduced this hyphomycetous genus. Phylogenetic analyses showed that Myrtapenidiella belongs in Teratosphaeriaceae (Quaedvlieg et al. 2014, Wijayawardene et al. 2014).

Myxospora L. Lombard \& Crous
Lombard et al. (2016) introduced this genus and showed that it belongs in Stachybotryaceae.

Neoacanthostigma Boonmee et al.

Boonmee et al. (2014) introduced this genus with a hyphomycetous asexual morph. Phylogenetic analyses showed that Neoacanthostigma belongs in Tubeufiaceae (Boonmee et al. 2014, Wijayawardene et al. 2014). Lu et al. (2017b) also introduced four new species with hyphomycetous asexual morphs and confirmed its placement in Tubeufiaceae.

Neoascochyta Q. Chen \& L. Cai

Chen et al. (2015) introduced this genus with coelomycetous asexual morph (ascochyta-like) and DNA sequence analyses showed that it belongs in Didymellaceae.

Neoascotaiwania Hern.-Restr. et al.

Hernández-Restrepo et al. (2017) introduced this genus with a hyphomycetous asexual morph.

Neobambusicola Crous \& M.J. Wingf.

Crous et al. (2014d) introduced this coelomycetous genus and showed that it belongs in Sulcatisporaceae.

Neocamarosporium Crous \& M.J. Wingf.

Crous et al. (2014c) showed that camarosporium-like taxa were distinct from Camarosporium sensu stricto and thus introduced Neocamarosporium. Wijayawardene et al. (2016) listed Neocamarosporium as a member of Pleosporaceae, Pleosporales. However, Wanasinghe et al. (2017) showed that this genus has distinct phylogenetic lineage in Pleosporales thus Neocamarosporiaceae was introduced.

Neocatenulostroma Quaedvl. \& Crous

Quaedvlieg et al. (2014) introduced this genus with a hyphomycetous asexual morph. Phylogenetic analyses showed that Neocatenulostroma belongs in Teratosphaeriaceae (Quaedvlieg et al. 2014, Wijayawardene et al. 2014). 
Neoceratosperma Crous \& Cheew.

Crous et al. (2014c) introduced this genus and mentioned that it has affinity with Mycosphaerellaceae. Wijayawardene et al. (2014) listed Neoceratosperma under Mycosphaerellaceae. Guatimosim et al. (2016) introduced two more species with mycosphaerella-like sexual morphs.

Neocercospora M. Bakhshi et al.

Bakshi et al. (2015) introduced Neocercospora and compared it with other cercosporoid-like genera. Molecular phylogenetic analyses showed that Neocercospora belongs in Mycosphaerellaceae.

Neocladophialophora Crous \& R.K. Schumach.

Crous et al. (2014d) introduced this monotypic hyphomycetous genus and compared it with Cladophialophora, Fusicladium and Polyscytalidium. Based on mega blast results of ITS sequence data, Crous et al. (2014d) mentioned that Neocladophialophora has a relationship with Triscelophorus cf. acuminatus.

Neocordana Hern.-Restr. \& Crous

Hernández-Restrepo et al. (2015) introduced this genus and molecular phylogenetic analyses showed that it belongs to Pyriculariaceae.

\section{Neocrinula Crous}

Crous et al. (2016a) introduced this hyphomycetous genus and accepted as a genus of Helotiaceae.

Neocucurbitaria Wanas. et al.

Wanasinghe et al. (2017) introduced this genus with coelomycetous asexual morph and showed that it belongs in Cucurbitariaceae.

Neocylindroseptoria Thambug. \& K.D. Hyde Thambugala et al. (2014) introduced this coelomycetous genus to accommodate Cylindroseptoria pistaciae Quaedvl. et al. which resided in Dothideaceae.

Neodactylaria Guevara-Suarez et al.

Crous et al. (2017) introduced this hyphomycetous genus and showed that it belongs in Dothideomycetes.
Neodeightonia C. Booth

Dai et al. (2016) reported on the holomorph of $N$. subglobosa C. Booth and confirmed its placement in Botryosphaeriaceae.

Neodeightoniella Crous \& W.J. Swart

Crous et al. (2013b) introduced this monotypic hyphomycetous genus and mega blast searches showed it has closer affinities to members in Mycosphaerellaceae.

\section{Neodevriesia Quaedvl. \& Crous}

Quaedvlieg et al. (2014) introduced this hyphomycetous genus to accommodate two Devriesia (Neodevriesiaceae) species which grouped as distinct to Devriesia sensu stricto in Teratosphaeriaceae. Crous et al. (2015) mentioned that Neodevriesia has teratosphaeria-like sexual morph.

\section{Neodidymelliopsis Q. Chen \& L. Cai}

Chen et al. (2015) introduced this genus with coelomycetous asexual morph and showed it belongs in Didymellaceae.

Neoeriomycopsis Crous \& M.J. Wingf.

Crous et al. (2015d) introduced this coelomycetous genus and showed that it belongs in Sordariomycetes, genera incertae sedis.

Neohelicomyces Z.L. Luo et al.

Luo et al. (2017) introduced this aquatic hyphomycetous genus and showed that it belongs in Tubeufiaceae.

Neohortaea Quaedvl. \& Crous

Quaedvlieg et al. (2014) introduced this monotypic hyphomycetous genus. Wijayawardene et al. (2014) listed it under Capnodiales, genera incertae sedis.

Neoidriella Hern.-Restr. \& Crous

Hernández-Restrepo et al. (2015) introduced this hyphomycetous genus. Molecular phylogenetic analyses showed that it belongs in Sordariomycetes, genera incertae sedis.

Neomicrosphaeropsis Thambug. et al. 
Thambugala et al. (2016) introduced this coelomycetous genus and showed that it belongs to Didymellaceae.

\section{Neomyrothecium L. Lombard \& Crous}

Lombard et al. (2016) introduced this genus with hyphomycetous asexual morph and showed that it belongs in Stachybotryaceae.

Neopenidiella Quaedvl. \& Crous

Quaedvlieg et al. (2014) introduced this monotypic hyphomycetous genus and showed that it belongs in Mycosphaerellaceae.

Neopestalotiopsis Maharachch. et al.

Maharachchikumbura et al. (2014) introduced this genus to accommodate several Pestalotiopsis species which do not cluster in Pestalotiopsis sensu stricto.

Neophaeococcomyces Crous \& M.J. Wingf. Crous et al. (2015e) introduced this genus and their phylogenetic analyses showed that it belongs in Strelitzianaceae.

Neophaeocryptopus Wanas. et al.

$\mathrm{Li}$ et al. (2016) introduced this genus with coelomycetous asexual morph.

Neophaeomoniella Rooney-Latham \& Crous Crous et al. (2015d) introduced this coelomycetous genus and showed that it belongs in Phaeomoniellales, genera incertae sedis. However, Index Fungorum (2015) introduced Phaeomoniellaceae and placed this genus.

Neophaeothecoidea Quaedvl. \& Crous

Quaedvlieg et al. (2014) introduced this monotypic hyphomycetous genus and showed that it belongs in Teratosphaeriaceae.

Neophysalospora Crous \& M.J. Wingf.

Crous et al. (2014d) introduced this genus with coelomycetous asexual morph and showed that it belongs to Clypeophysalosporaceae.

Neoplatysporoides Crous \& M.J. Wingf.

Crous et al. (2015d) introduced this genus with coelomycetous asexual morph and showed that it belongs to Pleosporaceae. Crous \&
Groenewald (2017) showed that this genus belongs in Libertasomycetaceae.

\section{Neopseudocercospora Crous}

Crous et al. (2013b) introduced this hyphomycetous genus and placed it in Mycosphaerellaceae based on mega-blast results. Braun et al. (2014) introduced the second species based on morphology.

\section{Neopseudocercosporella Videira \& Crous}

Videira et al. (2016) introduced this genus with hyphomycetous asexual morph. Phylogenetic analyses showed that it belongs in Mycosphaerellaceae.

\section{Neoscolecobasidium Crous}

Crous et al. (2016a) introduced this coelomycetous genus and confirmed its placement in Microthyriales genera incertae sedis.

Neopseudolachnella A. Hashim. \& Kaz. Tanaka

Hashimoto et al. (2015) introduced this genus and the phylogenetic analyses showed that it belongs in Chaetosphaeriaceae.

Neopyricularia Klaubauf et al.

Klaubauf et al. (2014) introduced this genus and showed that it belongs in Pyriculariaceae, Magnaporthales in their phylogenetic analyses.

Neoramichloridium Phook. et al.

Thambugala et al. (2017) introduced this hyphomycetous genus. Phylogenetic analyses showed that it belongs in Mycosphaerellaceae.

Neoseptoria Quaedvl. et al.

Quaedvlieg et al. (2013) introduced this coelomycetous genus and showed it belongs in Mycosphaerellaceae, but is distinct from Septoria sensu stricto.

Neoseptorioides Crous et al.

Crous et al. (2015e) introduced this genus and listed it under Saccharataceae.

Neosphaerellopsis Crous \& Trakun.

Trakunyingcharoen et al. (2014) introduced this coelomycetous genus and showed it belongs in Phaeosphaeriaceae. 
Neostagonospora Quaedvl. et al.

Quaedvlieg et al. (2013) introduced this genus and showed it belongs in Phaeosphaeriaceae. Phookamsak et al. (2014) confirmed this placement in their phylogenetic analyses.

Neostrelitziana Crous \& M.J. Wingf.

Crous et al. (2015d) introduced this hyphomycetous genus and showed it belongs to Chaetothyriales, genera incertae sedis.

Neosulcatispora Crous \& M.J. Wingf.

Crous et al. (2015e) introduced this coelomycetous genus and blast results showed that it has closer relationship to Vrystaatia in Phaeosphaeriaceae. Thus, we list Neosulcatispora under Phaeosphaeriaceae.

Neothyronectria Crous \& Thangavel

Crous et al. (2016a) introduced this coelomycetous genus and accepted as a genus belongs in Hypocreales, genera incertae sedis.

Neotorula Ariyaw. et al.

$\mathrm{Su}$ et al. (2016) introduced this genus and showed that it belongs to Torulaceae in their phylogenetic analyses.

Neotrimmatostroma Quaedvl. \& Crous

Quaedvlieg et al. (2014) introduced this genus with a hyphomycetous asexual morph. In phylogenetic analyses, Neotrimmatostroma resides in Teratosphaeriaceae.

Neotruncatella Hyang B. Lee \& T.T.T. Nguyen

Hyde et al. (2016) introduced this coelomycetous genus and showed that it belongs in Bartaliniaceae.

Neurospora Shear \& B.O. Dodge

Réblová et al. (2016a) reduced asexual typified name, Chrysonilia Arx under this genus.

Nothophoma Q. Chen \& L. Cai

Chen et al. (2015) introduced this genus and DNA sequence analyses showed that it belongs in Didymellaceae.

Occultibambusa D.Q. Dai \& K.D. Hyde
Dai et al. (2016) introduced this genus with coelomycetous asexual morph. Phylogenetic analyses showed that this genus belongs to Occultibambusaceae.

Ochroconis de Hoog \& Arx

Crous et al. (2015e) introduced Ochroconis pandanicola Crous \& M.J. Wingf. and mentioned Samerpitak et al. (2014) also accepted the placement of Ochroconis in Sympoventuriaceae.

Oleoguttula Selbmann \& de Hoog

The hyphomycetous genus Oleoguttula was introduced by Egidi et al. (2014) and belongs in Teratosphaeriaceae in their phylogenetic analysis.

Ophidiomyces Sigler et al.

Sigler et al. (2013) introduced a new genus to replace the hyphomycetous taxon, Chrysosporium ophiodiicola Guarro et al. which produces solitary, thallic conidia and arthric conidia.

Ophiodiaporthe Y.M. Ju et al.

$\mathrm{Fu}$ et al. (2013) introduced this genus and showed that it clustered in Diaporthaceae. Maharachchikumbura et al. (2015) agreed with this familial placement.

Paecilomyces Bainier

Zawadneak et al. (2015) confirmed the placement of this genus in Thermoascaceae.

Pallidocercospora Crous

Crous et al. (2013a) introduced this hyphomycetous genus to accommodate the cercospora-like taxa that group distinct to Cercospora sensu stricto in Mycosphaerellaceae.

Palmiascoma Phookamsak \& K.D. Hyde Liu et al. (2015) introduced this genus with a coelomycetous asexual morph and phylogenetic analyses showed that it belongs in Bambusicolaceae.

Parabambusicola Kaz. Tanaka \& K. Hiray. Tanaka et al. (2015) introduced this genus with a hyphomycetous (monodictys-like) asexual 
morph and phylogenetic analyses showed that it belongs to Parabambusicolaceae.

Parablastocatena Y.D. Zhang \& X.G. Zhang Zhang et al. (2012b) introduced this hyphomycetous genus but it lacks sequence data. Hence, taxonomic placement is uncertain.

Paraboeremia Q. Chen \& L. Cai

Chen et al. (2015) introduced this genus with coelomycetous asexual morph and DNA sequence analyses showed that it belongs in Didymellaceae.

Paracamarosporium Wijayaw. \& K.D. Hyde Wijayawardene et al. (2014c) introduced this genus to accommodate Camarosporium psoraleae Crous \& M.J. Wingf. which grouped (in Didymosphaeriaceae) away from Camarosporium sensu stricto in Pleosporinae.

\section{Paracladophialophora Crous}

Crous et al. (2016a) introduced this coelomycetous genus and confirmed its placement in Chaetothyriaceae.

Paracollema Otálora \& Wedin

Otálora et al. (2014) introduced this genus and placed it in Collemataceae.

\section{Paracremonium L. Lombard \& Crous}

Lombard et al. (2015) introduced this genus with hyphomycetous asexual morph. Molecular phylogenetic analyses showed that it belongs in Nectriaceae.

Paracylindrocarpon Crous et al.

Crous et al. (2016b) introduced this coelomycetous genus and accepted it as a genus in Bionectriaceae.

Paradendryphiella Woudenberg \& Crous Woudenberg et al. (2013) introduced Paradendryphiella and showed it resides in Pleosporaceae in their phylogenetic analyses. Ariyawansa et al. (2015c) accepted this placement in their phylogenetic analyses.

Paradiplococcium Hern.-Restr. et al.

Hernández-Restrepo et al. (2017) introduced this hyphomycetous genus and showed it belongs in Sordariomycetes.
Paracollema Otálora \& Wedin

Otálora et al. (2014) introduced this genus and placed it in Collemataceae.

Paradictyoarthrinium Matsush.

Doilom et al. (2016) showed that the hyphomycetous Paradictyoarthrinium has distinct phylogenetic lineage in Pleosporales, thus introduced Paradictyoarthriniaceae.

Paraepicoccum Matsush.

Thambugala et al. (2016) showed tht Paraepicoccum resides in Pleosporales based on molecular sequences of fresh collections.

Parafabraea Chen et al.

Chen et al. (2016) introduced this genus with coelomycetous asexual morph and showed that it belongs in Dermateaceae.

Parafuscosporella J. Yang \& K.D. Hyde

Yang et al. (2016) introduced this hyphomycetous genus and showed that it showed that belongs in Fuscosporellaceae.

Paragnomonia Senan. \& K.D. Hyde

Senanayake et al. (2017) introduced this genus with coelomycetous asexual morph and showed it belongs in Sydowiellaceae.

Paraidriella Hern.-Restr. \& Crous

Hernández-Restrepo et al. (2015) introduced this hyphomycetous genus. Molecular phylogenetic analyses showed that it belongs in Sordariomycetes, genera incertae sedis. Maharachchikumbura et al. (2016) accepted it as a genus in Xylariales.

Paraleptosphaeria Gruyter et al.

de Gruyter et al. (2012) introduced a new coelomycete genus to accommodate four species of Leptosphaeria spp. (L. dryadis, L. macrospora, $L$. nitschkei and $L$. praetermissa) and Phoma korfii. Phylogenetic analyses showed that Paraleptosphaeria resided in Leptosphaeriaceae. Ariyawansa et al. (2015b) also accepted the placement of Paraleptosphaeria in Leptosphaeriaceae.

Paramicrodochium Hern.-Restr. \& Crous 
Hernández-Restrepo et al. (2015) introduced this hyphomycetous genus and molecular phylogenetic analyses showed that it belongs in Sordariomycetes, genera incertae sedis.

Paramycoleptodiscus Crous \& M.J. Wingf.

Crous et al. (2016b) introduced this coelomycetous genus and accepted it as a genus in Acrospermales.

\section{Paramyrothecium L. Lombard \& Crous}

Lombard et al. (2016) introduced this genus with hyphomycetous asexual morph and showed that it belongs in Stachybotryaceae.

Paranannizziopsis Sigler et al.

Sigler et al. (2013) introduced this reptile pathogenic hyphomycetous genus with four species. Phylogenetic analyses showed that it belongs in Onygenaceae.

\section{Paraphaeomoniella Crous}

Crous et al. (2015d) introduced this genus and showed that it belongs in Phaeomoniellales, genera incertae sedis. However, Index Fungorum (2015) introduced Phaeomoniellaceae and placed this genus.

Parastagonospora Quaedvl. et al.

Quaedvlieg et al. (2013) introduced this genus which has stagonospora-like conidia and showed it belongs in Phaeosphaeriaceae. Phookamsak et al. (2014) and, Wijayawardene et al. (2014) confirmed this placement in their phylogenetic analyses.

Parawiesneriomyces Crous \& M.J. Wingf.

Crous et al. (2016b) introduced this hyphomycetous genus and accepted it as a genus in Wiesneriomycetaceae.

Parameliola Hongsanan et al.

$\mathrm{Li}$ et al. (2016a) introduced this hyphomycetous genus and showed that it belongs in Pleosporales.

\section{Parvothecium L. Lombard \& Crous}

Lombard et al. (2016) introduced this genus with hyphomycetous asexual morph and showed that it belongs in Stachybotryaceae.

Paulkirkia Wijayaw. et al.
Wijayawardene et al. (2016) introduced this genus and showed it belongs to Floricolaceae (current name Teichosporaceae).

\section{Perthomyces Crous}

Crous et al. (2017) introduced this coelomycetous genus and showed that it belongs in Pleosporales.

Perusta Egidi \& Stielow

Egidi et al. (2014) introduced this genus and showed that it grouped close to Dothideales in their phylogenetic analyses. However, we list Perusta as Dothideomycetes, genera incertae sedis.

Penidiellopsis Sandoval-Denis et al.

Crous et al. (2016b) introduced this hyphomycetous genus and accepted it as a genus in Teratosphaeriaceae.

Petrakia Syd. \& P. Syd.

Butin et al. (2013) stated that Petrakia showed Mycodidymella sexual morphs and resides in Pleosporales genera incertae sedis.

Petrophila de Hoog \& Quaedvl.

Egidi et al. (2014) introduced this hyphomycetous genus. Quaedvlieg et al. (2014) showed that it belongs in Extremaceae, Capnodiales.

Phaeomycocentrospora Crous et al.

Trakunyingcharoen et al. (2014) showed that this genus belongs in Didymellaceae in their phylogenetic analyses.

Phaeopoacea Thambug. et al.

Thambugala et al. (2017) introduced this genus with a coelomycetous asexual morph.

Phaeoschizotrichum Silva et al.

Silva et al. (2015) introduced this genus but sequence data is unavailable. Hence, taxonomic placement is uncertain.

Phaeothecoidiellaceae K.D. Hyde \& Hongsanan

Hongsanan et al. (2017b) introduced this family in Capnodiales and accepted two hyphomycetous genera as members in the family (viz. Houjia G.Y. Sun \& Crous and 
Phaeothecoidiella Batzer \& Crous along with one sexual morph viz. Chaetothyrina Theiss.

Phialemoniopsis Perdomo et al.

Perdomo et al. (2013) introduced Phialemoniopsis with sporodochium-like or pycnidium-like conidiomata. Phylogenetic analyses showed it does not group in any family, thus it was treated as Sordariomycetes, genera incertae sedis.

\section{Phomatodes Q. Chen \& L. Cai}

Chen et al. (2015) introduced this coelomycetous genus and DNA sequence analyses showed that it belongs in Didymellaceae.

Phragmocamarosporium Wijayaw. et al.

Wijayawardene et al. (2015) introduced this coelomycetous genus with two species and showed it belongs to Lentitheciaceae. This placement was confirmed by Wijayawardene et al. (2016).

\section{Platychora Petr.}

Hyde et al. (2016) reported a coelomycetous asexual morph in the culture of P. ulmi ( $\mathrm{J}$. Schröt) Petr. and confirmed the placement in Didymellaceae.

\section{Platytrachelon Réblová}

Réblová (2013) introduced this genus with hyphomycetous asexual morph. Zhang et al. (2017) showed that it belongs in Papulosaceae.

Pleiocarpon L. Lombard \& D. Aiello Aiello et al. (2017) introduced this hyphomycetous genus and showed that it belongs in Nectriaceae.

\section{Plenodomus Preuss}

de Gruyter et al. (2012) treated this genus as a distinct genus in Leptosphaeriaceae and showed that the type species of Plectophomella Moesz, P. visci (Sacc.) Moesz also resides in Plenodomus sensu stricto. Thus, Plectophomella was treated as a synonym of Plenodomus. Ariyawansa et al. (2015b) also accepted the placement of Plenodomus in Leptosphaeriaceae.
Pleomonodictys Hern.-Restr. et al.

Hernández-Restrepo et al. (2017) introduced this hyphomycetous genus and showed that it has distinct phylogenetic lineage in Dothideomycetes. Hence, they introduced Pleomonodictydaceae in Pleosporales.

Pleotrichocladium Hern.-Restr. et al.Hernández-Restrepo et al. (2017) introduced this hyphomycetous genus and showed that it belongs in Melanommataceae in their phylogenetic analyses.

Pleurophoma Höhn.

Crous et al. (2015d) introduced a new species and placed it in Lentitheciaceae.

Pleurophragmium Costantin

Dai et al. (2016) introduced P. bambusinum and showed that in belongs to Sordariomycetes genera incertae sedis.

Pleurotheciella Réblová et al.

Réblová et al. (2012) introduced this genus with a dactylaria-like asexual morph. Maharachchikumbura et al. (2016) listed this genus under Pleurotheciaceae.

Poaceicola W.J. Li et al.

Li et al. (2015) introduced this genus with coelomycetous asexual morph. Moreover, Li et al. (2015) showed that Poaceicola resides in Phaeosphaeriaceae.

Podosporiopsis Jian Ma et al.

$\mathrm{Ma}$ et al. (2016a) introduced this hyphomycetous genus. DNA sequences are unavailable thus placed in Ascomycota genera incertae sedis

Polyphialoseptoria Quaedvl. et al.

Quaedvlieg et al. (2013) introduced this coelomycetous genus which has septoria-like conidia. In phylogenetic analyses, it groups in Mycosphaerellaceae, but is distinct from Septoria sensu stricto.

\section{Polythrincium Kunze}

Wijayawardene et al. (2014c) treated Cymadothea F.A. Wolf as a synonym of Polythrincium and Rossman et al. (2015) accepted this. 
Porocercospora Amaradasa et al.

Amaradasa et al. (2014) introduced this genus to accommodate Cercospora seminalis Ellis \& Everh. which forms a distinct phylogenetic lineage in Pleosporaceae.

Prillieuxina G. Arnaud

Hongsanan et al. (2014) reduced the asexual typified name Leprieurina G. Arnaud under Prillieuxina.

\section{Proliferosphaera T.P. Devi}

Devi et al. (2012) introduced this monotypic, coelomycetous genus and compared it with other morphologically similar genera. The blast result of ITS sequence in GenBank indicate that it belongs in Dothideomycetes.

\section{Prosopidicola Crous \& C.L. Lennox}

Senanayake et al. (2017b) introduced Prosopidicolaceae to place this genus.

\section{Protostegia Cooke}

Crous et al. (2015a) showed that this genus belongs to Mycosphaerellaceae. They also designated an epitype for the type species.

Proxipyricularia Klaubauf et al.

Klaubauf et al. (2014) introduced this genus and showed that it belongs to Pyriculariaceae, Magnaporthales in their phylogenetic analyses.

Pseudoascochyta Valenzuela-Lopez et al.

Crous et al. (2016a) introduced this coelomycetous genus and showed that it is a member of Didymellaceae.

\section{Pseudoascotaiwania J. Yang et al.}

Yang et al. (2016) introduced this hyphomycetous genus and showed that showed that it belongs in Fuscosporellaceae.

Pseudoasteromassaria M. Matsum. \& Kaz. Tanaka

Ariyawansa et al. (2016a) introduced this genus with coelomycetous asexual morph and phylogenetic analyses show it belongs to Latoruaceae.

Pseudocamarosporium Wijayaw. \& K.D. Hyde
Wijayawardene et al. (2014c) introduced this coelomycete genus to accommodate five camarosporium-like taxa residing in Didymosphaeriaceae.

Pseudocatenomycopsis Crous \& L.A. Shuttlew. Crous et al. (2013b) introduced this monotypic, hyphomycetous genus and compared it with Catenomycopsis. Based on mega blast searches, Crous et al. (2013b) mentioned it has close relationship with Neofracchiaea. Maharachchikumbura et al. (2015) listed Pseudocatenomycopsis under Coronophorales, genera incertae sedis.

Pseudocoleodictyospora Doilom \& K.D. Hyde Doilom et al. (2017) introduced this hyphomycetous genus. Since it has distinct phylogenetic lineage in Dothideomycetes, Pseudocoleodictyosporaceae was introduced to accommodate the genus.

Pseudocoleophoma Kaz. Tanaka \& K. Hiray. Tanaka et al. (2015) introduced this genus with coelomycetous (coelophoma-like) asexual morph. Phylogenetic analyses showed that Pseudocoleophoma belongs in Dictyosporiaceae.

Pseudocosmospora C. Herrera \& P. Chaverri Herrera et al. (2013) introduced this genus with acremonium-like to verticillium-like asexual morphs. Further, Herrera et al. (2013b) showed that Pseudocosmospora belongs in Nectriaceae in their phylogenetic analyses.

Pseudocryptoclypeus A. Hashim. \& Kaz. Tanaka

Hashimoto et al. (2017) introduced this genus with a coelomycetous asexual morph. Phylogenetic analyses showed that it belongs in Lophiotremataceae.

Pseudodictyosporium Matsush.

Boonmee et al. (2016) showed that this genus belongs in Dictyosporiaceae.

Pseudodidymosphaeria Thambug. \& K.D. Hyde

Thambugala et al. (2015a) introduced this genus with coelomycetous asexual morph. 
Phylogenetic analyses showed that it belongs to Massarinaceae.

Pseudodinemasporium A. Hashim. \& Kaz. Tanaka

Hashimoto et al. (2015) introduced this genus and showed that it belongs in Chaetosphaeriaceae.

Pseudodiplococcium Hern.-Restr. et al. Hernández-Restrepo et al. (2017) introduced this hyphomycetous genus and showed it belongs in Xenospadicoidaceae.

Pseudohendersonia Crous \& M.E. Palm

Wijayawardene et al. (2016) introduced a new species and showed that it belongs to Didymellaceae.

\section{Pseudolachnella Teng}

Li et al. (2016a) introduced P. brevifusiformis A. Hashim. \& Kaz. Tanaka (coelomycetous) and showed that it belongs in Chaetosphaeriaceae.

Pseudomonodictys Doilom et al.

Ariyawansa et al. (2015a) introduced this hyphomycetous genus and showed that it belongs to Macrodiplodiopsidaceae.

Pseudopenidiella Crous \& Koukol

Crous et al. (2012c) introduced this genus and showed it to be distinct from Penidiella sensu stricto in Teratosphaeriaceae.

Pseudopestalotiopsis Maharachch. et al. 2014 Maharachchikumbura et al. (2014) introduced this genus to accommodate several Pestalotiopsis species which did not cluster in Pestalotiopsis sensu stricto. This placement was confirmed by Wijayawardene et al. (2016).

\section{Pseudophaeomoniella Nigro et al.}

Crous et al. (2015d) introduced this genus and showed that it belongs in Phaeomoniellales, genera incertae sedis. However, Index Fungorum (2015) introduced Phaeomoniellaceae and placed this genus.

Pseudophialophora J. Luo \& N. Zhang
Luo et al. (2015) introduced this genus and confirmed its placement in Magnaporthaceae in their phylogenetic analyses.

Pseudopithomyces Ariyaw. \& K.D. Hyde Ariyawansa et al. (2015a) introduced this hyphomycetous genus and showed that it belongs to Didymosphaeriaceae.

Pseudopyricularia Klaubauf et al.

Klaubauf et al. (2014) introduced this genus and showed that it belongs to Pyriculariaceae, Magnaporthales in their phylogenetic analyses.

Pseudoscopulariopsis M. Sandoval-Denis et al. Sandoval-Denis et al. (2016) introduced this genus to accommodate Scopulariopsis hibernica and Microascus schumacheri which group outside of Scopulariopsis sensu stricto and Microascus sensu stricto respectively.

Pseudosplanchnonema Chethana et al.

Chethana et al. (2015) introduced this genus with a phoma-like asexual morph, and showed that it belongs to Massarinaceae.

Pseudosporidesmium K.D. Hyde \& McKenzie $\mathrm{Su}$ et al. (2016) introduced this hyphomycetous genus and showed that it belongs in Xylariomycetidae genera incertae sedis.

Pseudotrichia Kirschst.

Liu et al. (2015) introduced P. rubriostiolata Phookamsak \& K.D. Hyde with a coelomycetous asexual morph and showed that it belongs to Melanommataceae in their phylogenetic analyses.

\section{Pseudoveronaea Crous \& Batzer}

Li et al. (2012) introduced this hyphomycetous genus and showed it belongs in Dissoconiaceae. Wijayawardene et al. (2014) also listed Pseudoveronaea in Dissoconiaceae.

Pseudoxylomyces Kaz. Tanaka \& K. Hiray. Tanaka et al. (2015) introduced this hyphomycetous genus and phylogenetic analyses showed that it belongs to Massrineae, genera incertae sedis.

Purpurepithecium Jayasiri \& K.D. Hyde 
Jayasiri et al. (2017) introduced this genus with hyphomycetous asexual morphs. In DNA sequences analyses, the genus resides in Gloniaceae.

Pustulomyces D.Q. Dai et al.

Notes: Dai et al. (2014c) introduced this bambusicolous coelomycetous genus and showed that it belongs in Diaporthaceae.

\section{Pyrenopeziza Fuckel}

Johnston et al. (2014) proposed to adopt sexual typified name Pyrenopeziza over Cylindrosporium Grev.

Queenslandipenidiella Quaedvl. \& Crous Quaedvlieg et al. (2014) introduced this hyphomycetous genus and in their phylogenetic analyses, showed it resides in Teratosphaeriaceae (Quaedvlieg et al. 2014).

\section{Querciphoma Crous}

Crous \& Groenewald (2017) introduced this coelomycetous genus and showed that it belongs in Leptosphaeriaceae.

Ramimonilia B. Stielow.\& Quaedvl.

Egidi et al. (2014) introduced this hyphomycetous genus and showed that it belongs in Capnodiales, genera incertae sedis.

Ramopenidiella Crous \& R.G. Shivas

Crous et al. (2014c) introduced this genus and listed it under Teratosphaeriaceae. Wijayawardene et al. (2014) accepted this familial placement.

Readerielliopsis Crous \& Decock

Crous et al. (2015d) introduced this coelomycetous genus and showed that it belongs to Capnodiaceae. However, Wijayawardene et al. (2016) showed that it belongs to Capnodiales, genera incertae sedis.

Rectifusarium L. Lombard

Lombard et al. (2015) introduced this genus with hyphomycetous asexual morph. Molecular phylogenetic analyses confirmed its placement in Nectriaceae.
Rhabdostromina Died.

Punithalingam \& Spooner (2011) reinstated the genus and treated Dendroseptoria Alcalde as a synonym.

Rhytidhysteron Speg.

Doilom et al. (2016) introduced Rhytidhysteron tectonae Doilom \& K.D. Hyde and showed that it belongs in Hysteriaceae.

\section{Roussoella Sacc.}

Liu et al. (2014), Dai et al. (2016) and Wijayawardene et al. (2016) accepted that this genus has coelomycetous asexual morphs (cytoplea-like).

Roussoellopsis I. Hino \& Katum.

Liu et al. (2014) accepted that this genus belongs in Roussoellaceae and showed melanconium-like asexual morphs.

Rupestriomyces Lei Su et al.

$\mathrm{Su}$ et al. (2015) introduced this genus and phylogenetic analyses showed that it belongs to Dothideomycetes, genera incertae sedis.

Ruptoseptoria Quaedvl. et al.

Quaedvlieg et al. (2013) introduced this coelomycetous genus which has septoria-like conidia. In phylogenetic analyses, it groups in Mycosphaerellaceae but is distinct to Septoria sensu stricto and Polyphialoseptoria.

Rutola J.L. Crane \& Schokn.

Crous et al. (2015a) showed that this genus belongs in Pleosporales, genera incertae sedis. They also designated an epitype for the type species.

Sabahriopsis Crous \& M.J. Wingf.

Crous et al. (2015d) introduced this genus and listed it in Helotiales, genera incertae sedis.

Saccothecium Fr.

Li et al. (2016a) introduced S. rubi Jayasiri et al. with an asexual morph which has sporodochial to acervular conidiomata.

Sakireeta Subram. \& K. Ramakr. 
Crous et al. (2015b) showed that this genus belongs to Botryosphaeriaceae and has a distinct phylogenetic lineage to Tiarosporella.

Sardiniella Linaldeddu et al.

Linaldeddu et al. (2016) introduced this coelomycetous genus and phylogenetic analyses confirmed its placement in Botryosphaeriaceae.

Sativumoides S.C. Ren et al.

Ren et al. (2012) introduced this monotypic, hyphomycetous genus, but sequence data is unavailable. Hence, taxonomic placement is uncertain.

Saxomyces L. Selbmann \& D. Isola

Selbmann et al. (2014) introduced this genus but it was invalidly published (Art. 40.1) thus Wijayawardene et al. (2017) validated the genus.

Saxophila Selbmann \& de Hoog

Isola et al. (2016) introduced this hyphomycetous genus and showed that it belongs in Extremaceae.

\section{Scleropezicula Verkley}

Johnston et al. (2014) proposed to adopt sexual typified name Scleropezicula over Cryptosympodula Verkley

Scolecobeltrania Iturr. et al.

Iturriaga et al. (2013) introduced this monotypic, hyphomycetous genus. Sequence data are unavailable, thus taxonomic placement is uncertain and it is presently accommodated in Ascomycota, genera incertae sedis.

Seifertia Partr. \& Morgan-Jones

Li et al. (2016b) introduced S. shangrilaensis and showed it belongs in Melanommataceae.

Seimatosporium Corda

Perera et al. (2016) introduced sexual morph of S. cornii.

Septoriella Oudem.

Crous et al. (2015a) reduced Wojnowicia under Septoriella as latter name is the oldest name.
Quaedvlieg et al. (2013) introduced this coelomycetous genus which has septoria-like conidia, and in their phylogenetic analyses showed that Septorioides belongs in Botryosphaeriaceae. However, Wyka and Broders (2016) showed that Septorioides has distinct phylogenetic lineage in Botryosphaeriales thus introduced Septorioideaceae.

Seriascoma Phookamsak et al.

Dai et al. (2016) introduced this genus with coelomycetous asexual morph. Phylogenetic analyses showed that this genus belongs to Occultibambusaceae.

Setophaeosphaeria Crous \& Y. Zhang ter Crous et al. (2014c) introduced this genus with a coelomycetous asexual morph and listed it under Phaeosphaeriaceae. However, Phookamsak et al. (2014) showed that Setophaeosphaeria grouped outside Phaeosphaeriaceae thus, threated the genus in Pleosporales, genera incertae sedis.

Setoseptoria Quaedvl. et al.

Quaedvlieg et al. (2013) introduced this coelomycetous genus and showed it belongs in Lentitheciaceae. Wijayawardene et al. (2014) also agreed with this familial placement.

Sheathnema Dubey \& Moonambeth

Dubey \& Moonnambeth (2014) introduced this genus. DNA sequence data unavailable, thus placed in Ascomycota, genera incertae sedis.

Shrungabeeja V.G. Rao \& K.A. Reddy Ariyawansa et al. (2015a) introduced $S$. longiappendiculata Sommai et al. and showed that it belongs in Tetraplosphaeriaceae.

Sigleria Hirooka et al.

Hirooka et al. (2016) introduced this hyphomycetous genus and showed that it belongs to Spiromastigaceae in their phylogenetic analyses.

Simmonsiella J.L. Crane \& A.N. Mill.

Crane \& Miller (2016) introduced this genus and compared it with other torula-like hyphomycetous genera. DNA sequence data

Septorioides Quaedvl. et al. 
are unavailable thus, taxonomic placement is uncertain.

Sirastachys L. Lombard \& Crous

Lombard et al. (2016) introduced this genus and showed that it belongs in Stachybotryaceae.

Slopeiomyces Klaubauf et al.

Klaubauf et al. (2014) introduced this genus with a hyphomycetous asexual morph and showed that it belongs in Magnaporthaceae, Magnaporthales in their phylogenetic analyses.

Smaragdiniseta L. Lombard \& Crous

Lombard et al. (2016) introduced this genus with hyphomycetous asexual morph and showed that it belongs in Stachybotryaceae.

Sodiomyces Grum-Grzhimaylo et al.

Grum-Grzhimaylo et al. (2013) introduced this genus with hyphomycetous asexual morph.

Soloacrosporiella Crous \& M.J. Wingf.

Crous et al. (2015d) introduced this monotypic genus and listed it under Dothideomycetes, genera incertae sedis.

Soosiella Hujslová \& M. Kolařík

Hujslová et al. (2014) introduced this hyphomycetous genus and showed that it belongs in Helotiales.

Sparticola Phukhams. et al.

Phukhamsakda et al. (2016a) introduced this genus with hyphomycetous asexual morph and showed that it belongs to Sporormiaceae.

Spissiomyces Lei Su et al.

$\mathrm{Su}$ et al. (2015) introduced this genus and phylogenetic analyses showed that it belongs to Dothideomycetes, genera incertae sedis.

Sporidesmioides Li et al.

$\mathrm{Li}$ et al. (2016c) introduced this hyphomycetous genus and showed that it belongs in Torulaceae

Sporidesmium Link
Zhang et al. (2017) reported the sexual morph of Sporidesmium and introduced $S$. thailandense W. Dong et al.

Stagonosporopsis Died.

Hyde et al. (2016) introduced S. centaureae Tennakoon et al. (sexual morph) which groups in Stagonosporopsis s. str.

\section{Staurosphaeria Rabenh.}

Wanasinghe et al. (2017) showed that this genus belongs in Coniothyriaceae and treated Hazslinszkyomyces Crous \& R.K. Schumach. as a synonym (Crous \& Groenewald 2017).

Stigmatodiscus Voglmayr \& Jaklitsch

Voglmayr et al. (2016) introduced this genus with coelomycetous asexual morph.

Strelitziana Arzanlou \& Crous

Crous et al. (2015e) introduced the new family, Strelitzianaceae Crous \& M.J. Wingf. to place this genus.

\section{Striatibotrys L. Lombard \& Crous}

Lombard et al. (2016) introduced this genus with hyphomycetous asexual morph and showed that it belongs in Stachybotryaceae.

Striaticonidium L. Lombard \& Crous

Lombard et al. (2016) introduced this genus with hyphomycetous asexual morph and showed that it belongs in Stachybotryaceae.

Stromatoseptoria Quaedvl. et al.

Quaedvlieg et al. (2013) introduced this coelomycetous genus and showed that it groups in Mycosphaerellaceae. However, Stromatoseptoria is distinct from Septoria sensu stricto and Polyphialoseptoria which also resides in the same family.

Subplenodomus Gruyter et al.

de Gruyter et al. (2012) introduced this coelomycetous genus and transferred four Phoma species (P. apiicola, P. drobnjacensis, $P$. valerianae and $P$. violicola) to new genus. Ariyawansa et al. (2015b) also accepted the placement of Subplenodomus in Leptosphaeriaceae. 
Sulcatispora Kaz. Tanaka \& K. Hiray.

Tanaka et al. (2015) introduced this genus with coelomycetous asexual morph. Phylogenetic analyses showed that it belongs to Sulcatisporaceae.

Superstratomyces van Nieuwenhuijzen et al. van Nieuwenhuijzen et al. (2016) introduced this coelomycetous genus. In their phylogenetic analyses, van Nieuwenhuijzen et al. (2016) showed that the genus formed a distinct lineage and thus was introduced in Superstratomycetales and Superstratomycetaceae in Dothideomycetes.

Suttonomyces Wijayaw. et al.

Wijayawardene et al. (2015) introduced this genus with two species and showed it belongs to Massarinaceae. This placement was confirmed by Wijayawardene et al. (2016).

Sympodiosynnema J.W. Xia \& X.G. Zhang

$\mathrm{Xia}$ et al. (2016) introduced this hyphomycetous genus. DNA sequences lack thus tqxonomic placement is uncertain.

Syncarpella Theiss. \& Syd.

Wijayawardene et al. (2017) treated Syntholus A.W. Ramaley \& M.E. Barr as synonym of Syncarpella.

Tamaricicola Thambug. et al.

Thambugala et al. (2016) introduced this genus with coelomycetous asexual morph. Phylogenetic analyses showed that it belongs to Pleosporaceae.

Tamhinispora K.C. Rajeshk. \& Rahul Sharma Rajeshkumar \& Sharma (2013) introduced this monotypic, hyphomycetous genus and showed it belongs to Tubeufiaceae in their phylogenetic analyses. Boonmee et al. (2014) also agreed with this familial placement.

Tangerinosporium L. Lombard \& Crous Lombard et al. (2016) introduced this genus with hyphomycetous asexual morph and showed that it belongs in Stachybotryaceae.

Tenuiappendicula Senan. et al.
Senanayake et al. (2017) introduced this genus with a coelomycetous asexual morph and showed it belongs in Sydowiellaceae.

Teratoramularia Videira et al.

Videira et al. (2016) introduced this hyphomycetous genus. Phylogenetic analyses showed that it belongs in Teratosphaeriaceae.

Thailandiomyces Pinruan et al.

Suetrong et al. (2015) stated that Thailandiomyces bisetulosus Pinruan et al. (the type species of Thailandiomyces) has Craspedodidymum licualae asexual morph.

Thermothelomyces Y. Marín et al.

Marin-Felix et al. (2015) introduced tis genus with hyphomycetous asexual morph. Phylogenetic analyses showed that Thermothelomyces belongs in Chaetomiaceae.

Ticosynnema R.F. Castañeda et al.

Castañeda-Ruiz et al. (2012a) introduced this monotypic, hyphomycetous genus, but sequence data is unavailable. Hence, the genus was listed under Ascomycota, genera incertae sedis.

Tirisporella E.B.G. Jones et al.

Suetrong et al. (2015) stated that $T$. beccariana (Ces.) E.B.G. Jones et al. (the type species of Tirisporella) has Phialophora $c f$. olivaceae asexual morph.

\section{Torulaceae Corda}

$\mathrm{Li}$ et al. (2017b) revisited the family and introduced several new species of Torula based on morpho-molecular analyses.

Towyspora Wanas. et al.

$\mathrm{Li}$ et al (2016a) introduced this genus with coelomycetous asexual morph and phylogenetic analyses showed that it belongs to Lentitheciaceae.

\section{Trematosphaeria Fuckel}

Kirk et al. (2008) reported zalerion-like asexual morphs and Hyde et al. (2013) reported hyphopodia-like structures from the cultures of this genus. 
Tretolylea Cantillo et al.

Cantillo-Pérez et al. (2016) introduced this hyphomycetous genus.

Triadelphia Shearer \& J.L. Crane

Crous et al. (2015d) introduced a new species, $T$. disseminata Madrid \& J. Edathodu and showed that it belongs to Microascales, genera incertae sedis.

\section{Trimmatostroma Corda}

Ekanayake et al. (2017) treated this genus belongs in Mollisiaceae.

Trinosporium Crous \& Decock

Crous et al. (2012c) introduced this coelomycetous genus and mega blast results showed that it belongs in Xylonomycetaceae.

Tristratiperidium Daranagama et al.

Daranagama et al. (2016) introduced this genus with hyphomycetous asexual morph.

Tubakia B. Sutton

Senanayake et al. (2017b) accepted the genus as a member in Melanconiellaceae.

Tubeufia Penz. \& Sacc.

Hyde et al. (2016) introduced T. hyalospora Y.Z. Lu et al. with a hyphomycetous asexual morph. Lu et al. (2017a) also introduced three Tubeufia species with hyphomycetous asexual morphs

Tubeufiales Boonmee \& K.D. Hyde

Boonmee et al. (2014) revised the family Tubeufiaceae and introduced Tubeufiales. Further the family was revisited.

Tylophoron Nyl. ex Stizenb. (= Blarneya D. Hawksw et al.)

Ertz et al. (2011) synonymised the asexual typified name Blarneya under the sexual typified name Tylophoron, based on phylogenetic analyses.

Uleothyrium Petr.

Hongsanan et al. (2014) treated Septothyrella Höhn. as a synonym of this genus.

Umthunziomyces Crous \& M.J. Wingf.
Crous et al. (2016a) introduced this coelomycetous genus and confirmed its placement in Planistromellaceae.

Uwemyces Hern.-Restr. et al.

Notes: Crous et al. (2016b) introduced this hyphomycetous genus and accepted it as a genus in Mycosphaerellaceae.

Valsaria Ces. \& De Not.

Jaklitsch et al. (2015) accepted this with coelomycetous asexual morphs. Further, Jaklitsch et al. (2015) showed that Valsaria has distinct phylogenetic placement in Dothideomycetes. Hence, Valsariaceae, Valsariales were introduced.

Vamsapriya Gawas \& Bhat

Dai et al. (2014a) introduced $V$. bambusicola D.Q. Dai et al. and V. khunkonensis D.Q. Dai et al. and showed that these species reside in Xylariaceae.

Varicosporella Lechat \& J. Fourn.

Lechat \& Fournier (2015) introduced this genus with fusarium-like asexual morph and phylogenetic analyses showed that it belongs in Nectriaceae.

Verkleyomyces Y. Marín \& Crous

Marin-Felix et al. (2016) introduced this genus with coelomycetous asexual morph. The genus accommodated in Dermateaceae in their phylogenetic analyses.

\section{Vermiconia Egidi \& Onofri}

Egidi et al. (2014) introduced this hyphomycetous genus and showed it belongs in Teratosphaeriaceae sensu lato. In the phylogenetic analyses of Quaedvlieg et al. (2014) Vermiconia resided in Extremaceae.

\section{Verrucoconiothyrium Crous}

Crous et al. (2015c) introduced this coelomycetous genus and showed it belongs to Didymosphaeriaceae. This familial placement was confirmed by Wijayawardene et al. (2016).

Verruconis Samerp. et al.

Samerpitak et al. (2014) introduced Verruconis with placement in Sympoventuriaceae.

Vikalpa D'souza et al. 
Boonmee et al. (2016) introduced this hyphomycetous genus and showed that it belongs in Dictyosporiaceae

Vrystaatia Quaedvl. et al. Quaedvlieg et al. (2013) introduced this monotypic, coelomycetous genus and their phylogenetic analyses showed that it groups in Phaeosphaeriaceae. Phookamsak et al. (2014) agreed with this familial placement.

Wiesneriomyces Koord.

See under Wiesneriomycetaceae.

Wiesneriomycetaceae Suetrong et al.

Suetrong et al. (2014) showed that it has distinct phylogenetic lineage in Pleosporales, thus introduced new family Wiesneriomycetaceae. Index Fungorum (2017) listed Wiesneriomycetaceae as an invalid family thus Wijayawardene et al. (2017) validate it with relevant corrections.

\section{Wojnowiciella Crous et al.}

Crous et al. (2015d) introduced this genus and showed that it belongs to Phaeosphaeriaceae. Wijayawardene et al. (2016) agreed with this placement.

Woswasia Jaklitsch et al.

Jaklitsch et al. (2013) introduced this genus with hyphomycetous asexual morphs. Zhang et al. (2017) introduced Woswasiaceae to accommodate this genus.

Xenoacremonium L. Lombard \& Crous

Lombard et al. (2015) introduced this genus with hyphomycetous asexual morph and phylogenetic analyses showed that it belongs in Nectriaceae.

Xenocamarosporium Crous \& M.J. Wingf.

Crous et al. (2015d) introduced this coelomycetous genus and showed it belongs to Didymosphaeriaceae. Wijayawardene et al. (2016) accepted this familial placement.

Xenodidymella Q. Chen \& L. Cai

Chen et al. (2015) introduced this genus with a coelomycetous asexual morph and DNA sequence analyses showed that it belongs in Didymellaceae.
Xenoleptographium Marinc. et al.

Crous et al. (2015e) introduced this genus and showed that it belongs to Nectriaceae in their phylogenetic analyses.

Xenomyrothecium L. Lombard \& Crous

Lombard et al. (2016) introduced this genus with hyphomycetous asexual morph and showed that it belongs in Stachybotryaceae.

Xenopenidiella Quaedvl. \& Crous

Quaedvlieg et al. (2014) introduced this hyphomycetous genus. In their phylogenetic analyses, Xenopenidiella resided in Teratosphaeriaceae (Quaedvlieg et al. 2014).

Xenopyricularia Klaubauf et al.

Klaubauf et al. (2014) introduced this hyphomycetous genus and showed that it belongs in Pyriculariaceae, Magnaporthales in their phylogenetic analyses.

Xenoramularia Videira et al.

Videira et al. (2016) introduced this hyphomycetous genus and in phylogenetic analyses, they showed that it belongs in Mycosphaerellaceae

Xenoseptoria Quaedvl. et al.

Quaedvlieg et al. (2013) introduced this monotypic, coelomycetous genus and showed that it groups in Phaeosphaeriaceae. Phookamsak et al. (2014) agreed with this familial placement.

\section{Xenosonderhenia Crous}

Crous et al. (2012c) introduced this coelomycetous genus and showed that it belongs in Capnodiales, genera incertae sedis.

Xenospadicoides Hern.-Restr. et al.

Hernández-Restrepo et al. (2017) introduced this hyphomycetous genus and showed it has distinct phylogenetic lineage in Sordariomycetes. Thus, Xenospadicoidales and Xenospadicoidaceae were introduced.

\section{Xenostigmina Crous}

Phookamsak et al. (2014) showed Xenostigmina belongs to Melanommataceaein their molecular phylogenetic analyses. Crous et 
al. (2013a) designated the epitype and exepitype culture.

Xerombrophila Baral

Baral et al. (2013) introduced this genus with hyphomycetous asexual morph. DNA sequence analyses showed that it belongs to Helotiaceae.

Xiuguozhangia K. Zhang et al.

Zhang et al. (2014) introduced this genus to accommodate five Piricaudiopsis species, but characterized by holoblastic conidial ontogeny. Sequence data is unavailable thus, taxonomic placement is uncertain.

Xyladictyochaeta Hern.-Restr. et al.

Hernández-Restrepo et al. (2017) introduced this hyphomycetous genus and showed that it belongs in Xylariales in their phylogenetic analyses.

Xylochrysis Réblová et al.Réblová et al. (2014) introduced this genus with hyphomycetous asexual morph. Zhang et al. (2017) placed the genus in Woswasiaceae based on their phylogenetic analyses.

Ypsilomyces D.A.C. Almeida \& Gusmão Almeida \& Gusmão (2014) introduced this hyphomycetous genus, but it lacks sequence data. Hence, taxonomic placement is uncertain.Zeloasperisporium R.F. Castañeda Crous et al. (2015e) introduced two species and showed that both reside in Zeloasperisporiaceae. Hongsanan et al. (2015) introduced new order to place Zeloasperisporiaceae.

Zelodactylaria A.C. Cruz et al.

Da Cruz et al. (2012) introduced this monotypic, hyphomycetous genus. Sequence data are unavailable; thus, the taxonomic placement is uncertain. 


\section{Acknowledgements}

We would like to thank Dr. Cletus P. Kurtzman for his valuable suggestions on genera in Saccharomycetes. This study was jointly funded by the Youth Research Fund of Shandong Academy of Agricultural Sciences (2016YQN32), the Science and Technology Program of Tai'an, China (201540699), and Agricultural science and technology innovation project of Shandong Academy of Agricultural Sciences (CXGC2016B07).

\section{References}

Abdel-Wahab MA, Dayarathne MC, Suetrong S, Guo SY et al. 2017 - New saprobic marine fungi and a new combination. Botanica Marina. DOI: https://doi.org/10.1515/bot-2016-0118

Almeida DAC, Gusmão LFP. 2014 - Ypsilomyces, a new thallic genus of conidial fungi from the semi-arid Caatinga biome of Brazil. Mycotaxon 129, 181-186.

Amaradasa BS, Madrid H, Groenewald JZ, Crous PW et al. 2014 - Porocercospora seminalisgen et comb. nov., the causal organism of buffalograss false smut. Mycologia 106(1), 77-85.

Ariyawansa HA, Hyde KD, Jayasiri SC, Buyck B et al. 2015a - Fungal diversity notes 111-252 taxonomic and phylogenetic contributions to fungal taxa. Fungal Diversity 75, 1-248.

Ariyawansa HA, Phukhamsakda C, Thambugala KM, Bulgakov TS, et al. 2015b Revision and phylogeny of Leptosphaeriaceae. Fungal Diversity. 74, 19-51.

Ariyawansa HA, Tanaka K, Thambugala KM, Phookamsak R et al. 2014 - A molecular phylogenetic reappraisal of the Didymosphaeriaceae (= Montagnulaceae). Fungal Diversity 68, 69-104.

Ariyawansa HA, Thambugala KM, Manamgoda DS, Jayawardena R et al. 2015c - Towards a natural classification and backbone tree for Pleosporaceae. Fungal Diversity 71, 85-139.

Augustin JO, Groenewald JZ, Nascimento RJ, Mizubuti ESG et al. 2012 - Yet more Bweeds in the garden: fungal novelties from nests of leaf-cutting ants. PLoS ONE 8, e82265.

Bakhshi M, Arzanlou M, Babai-Ahari A, Groenewald JZ. 2015 - Is morphology in Cercospora a reliable reflection of generic affinity? Phytotaxa 213, 22-34.

Baral HO, Marson G, Bogale M, Untereiner WA. 2013 - Xerombrophila crystallifera, a new genus and species in the Helotiales. Mycological Progress 12, 475-488.

Baral H-O, Weber E, Stadler M, Gams W. 2016 - Recommendations about genera to be protected or suppressed in the Orbiliaceae (Orbiliomycetes).Mycological Progress doi:10.1007/s11557017-1300-6.

Baschien C, Tsui CK, Gulis V, Szewzyk U et al. 2013 - The molecular phylogeny of aquatic hyphomycetes with affinity to the Leotiomycetes. Fungal Biology 117, 660-672.

Beier GL, Hokanson SC, Bates ST, Blanchette RA. 2015 - Aurantioporthe corni gen. et comb. nov., an endophyte and pathogen of Cornus alternifolia. Mycologia 107, 66-79.

Boonmee S, D'souza MJ, Luo Z, Pinruan U et al. 2016 - Dictyosporiaceae fam. nov. Fungal Diversity 80, 457-482.

Boonmee S, Rossman AY, Liu JK, Li WJ et al. 2014 - Tubeufiales, ord. nov., integrating sexual and asexual generic names. Fungal Diversity 68, 239-298.

Bose T, Reynolds DR, Berbee ML. 2014 - Common, unsightly and until now undescribed: Fumiglobus pieridicola sp. nov., a sooty mold infesting Pieris japonica from western North America. Mycologia 106, 746-756.

Brahmanage RS, Lu YZ, Bhat DJ, Wanasinghe DN et al. 2017 - Phylogenetic investigations on freshwater fungi in Tubeufiaceae (Tubeufiales) reveals the new genus Dictyospora and new species Chlamydotubeufia aquatica and Helicosporium flavum. Mycosphere 8, 917-933.

Braun U, Crous PW, Nakashima C. 2013 - Cercosporoid fungi (Mycosphaerellaceae) 2. Species on monocots (Acoraceae to Xyridaceae, excluding Poaceae). IMA fungus 5, 203-390.

Butin H, Holdenrieder O, Sieber TN. 2013 - The complete life cycle of Petrakia echinata. Mycological Progress 12, 427-435. 
Cannon PF, Buddie AG, Bridge PD, de Neergaard E et al. 2012 - Lectera, a new genus of the Plectosphaerellaceae for the legume pathogen Volutella colletotrichoides. MycoKeys 3, 23-36.

Cantillo-Pérez T, Gusmão LF, Castañeda-Ruiz RF. 2016 - Tretolylea, a new genus from the Brazilian semiarid region. Mycotaxon 130, 977-981.

Carmo LT, Santana JM, Gusmão LFP, Sótão HMP et al. 2014 - Anabahusakala, a new genus from the Brazilian Amazon rainforest. Mycotaxon 127, 11-15.

Castañeda-Ruiz RF, Granados MM, Mardones M, Stadler M et al. 2012a - A microfungus from Costa Rica: Ticosynnema gen. nov. Mycotaxon 122, 255-259.

Castañeda-Ruiz RF, Heredia GA, Arias RMM, Stadler M et al. 2012b - Two new fungi from Mexico: Anaseptoidium gen. nov. and Cylindrosympodium sosae sp. nov. Mycotaxon 119, 141-148.

Castañeda-Ruiz RF, Hernández-Restrepo M, Gené J, Guarro J et al. 2013 - Two new microfungi from Portugal: Magnohelicospora iberica gen. \& sp. nov. and Phaeodactylium stadleri sp. nov. Mycotaxon 121, 171-179.

Castañeda-Ruiz RF, Leão-Ferreira SM, Gusmão LFP. 2015 - Distophragmia, a new genus of microfungi to accommodate Endophragmiella rigidiuscula. Mycotaxon 130, 499-503.

Checa J, Jaklitsch WM, Blanco MN, Moreno G et al. 2015 - Two new species of Thyronectria from Mediterranean Europe. Mycologia 107, 1314-1322.

Chen C, Verkley GJ, Sun G, Groenewald JZ et al. 2016 - Redefining common endophytes and plant pathogens in Neofabraea, Pezicula, and related genera. Fungal Biology 120, 1291-1322.

Chen Q, Jiang JR, Zhang GZ, Cai L, Crous PW. 2015 - Resolving the Phoma enigma. Studies in Mycology 82, 137-217.

Chen SF, Wingfield MJ, Roux J. 2013 - Diversimorbus metrosiderotis gen. et sp. nov. and three new species of Holocryphia (Cryphonectriaceae) associated with cankers on native Metrosideros angustifolia trees in South Africa. Fungal Biology 117, 289-310.

Chethana T, Liu M, Ariyawansa HA, Konta S, Wanasinghe DN et al. 2015 - Splanchnonema-like species in Pleosporales: introducing Pseudosplanchnonema gen. nov. in Massarinaceae. Phytotaxa 231, 133-44.

Choi IY, Kim BS, Park JH, Cho SE, Shin HD. 2014 - First report of black stem caused by Botryosporium longibrachiatum on statice in Korea. Plant Disease 98, 1431.

Costa PM, Barbosa MA, Malosso E, Castañeda-Ruiz RF. 2016 - Anaverticicladus uncinatus gen. \& sp. nov. from decaying leaves from Brazil. Mycotaxon 131, 687-691.

Crane C, Burgess TI. 2013 - Luteocirrhus shearii gen. sp. nov.(Diaporthales, Cryphonectriaceae) pathogenic to Proteaceae in the south Western Australian Floristic Region. IMA fungus 4, $111-122$.

Crane JL, Miller AN. 2016 - Studies in genera similar to Torula: Bahusaganda, Bahusandhika, Pseudotorula, and Simmonsiella gen. nov. IMA fungus 7, 29-45.

Crous PC, Shivas RG, Wingfield MJ, Summerell BA et al. 2012a. Fungal Planet description sheets: 128-153. Persoonia. 29, 146-201.

Crous PW, Braun U, Hunter GC, Wingfield MJ et al. 2013a - Phylogenetic lineages in Pseudocercospora. Studies in Mycology 75, 37-114.

Crous PW, Carris LM, Giraldo A, Groenewald JZ et al. 2015a - The Genera of Fungi - fixing the application of the type species of generic names - G 2: Allantophomopsis, Latorua, Macrodiplodiopsis, Macrohilum, Milospium, Protostegia, Pyricularia, Robillarda, Rotula, Septoriella, Torula, and Wojnowicia. IMA fungus 6, 163-198.

Crous PW, Giraldo A, Hawksworth DL, Robert V et al. 2014a The Genera of Fungi: fixing the application of type species of generic names. IMA fungus 5, 141-160.

Crous PW, Müller M, Sánchez RM, Giordano L et al. 2015b - Resolving Tiarosporella spp. allied to Botryosphaeriaceae and Phacidiaceae. Phytotaxa 202, 73-93. 
Crous PW, Quaedvlieg W, Hansen K, Hawksworth DL et al. 2014b - Phacidium and Ceuthospora (Phacidiaceae) are congeneric: taxonomic and nomenclatural implications. IMA fungus 5, 173-193.

Crous PW, Schumacher RK, Wingfield MJ, Lombard L et al. 2015c - Fungal Systematics and Evolution: FUSE 1. Sydowia 67, 81-118.

Crous PW, Shivas RG, Quaedvlieg W, van der Bank M et al. 2014c - Fungal Planet Description Sheets: 214-280. Persoonia. 32, 184-306.

Crous PW, Summerell BA, Alfenas AC, Edwards J et al. 2012b - Genera of diaporthalean coelomycetes associated with leaf spots of tree hosts. Persoonia 28, 66-75.

Crous PW, Summerell BA, Shivas RG, Burgess TI et al. 2012c - Fungal Planet Description Sheets, 107-127. Persoonia 28, 138-182.

Crous PW, Verkley GJM, Christensen M, Castañeda-Ruiz RF et al. 2012d - How important are conidial appendages? Persoonia 28, 126-137.

Crous PW, Wingfield MJ, Burgess TI, Hardy GS et al. 2016a - Fungal Planet description sheets: 469-557; Persoonia 37, 218-403.

Crous PW, Wingfield MJ, Burgess TI, Hardy GS et al. 2017 - Fungal Planet description sheets: 558-624. Persoonia 38, 240-384.

Crous PW, Wingfield MJ, Guarro J, Cheewangkoon R et al. 2013b - Fungal Planet description sheets: 154-213; Persoonia 31, 188-296.

Crous PW, Wingfield MJ, Guarro J, Hernández-Restrepo M et al. 2015d - Fungal Planet Description Sheets: 320-370; Persoonia 34, 167-266.

Crous PW, Wingfield MJ, Le Roux JJ, Richardson DM et al. 2015e - Fungal Planet Description Sheets, 371-399; Persoonia 35, 264-327.

Crous PW, Wingfield MJ, Richardson DM, Le Roux JJ et al. 2016b - Fungal Planet description sheets, 400-468. Persoonia: Molecular Phylogeny and Evolution of Fungi, 36, 316.

Crous PW, Wingfield MJ, Schumacher RK, Summerell BA et al. 2014d - Fungal Planet Description Sheets, 281-319; Persoonia 33, 212-289.

Da Cruz RAC, Gusmão LFP, Castañeda Ruiz RF, Stadler M, et al. 2012 - Zelodactylaria, an interesting new genus from semi-arid northeast Brazil. Mycotaxon 119, 241-248.

da Silva M, Pinho DB, Pereira OL, Fernandes FM, Barreto RW. 2016 - Naming potentially endangered parasites: foliicolous mycobiota of Dimorphandra wilsonii, a highly threatened Brazilian tree species. PloS one. 11, e0147895.

Dai DQ, Bahkali AH, Li QR, Bhat DJ et al. 2014a - Vamsapriya (Xylariaceae) re-described, with two new species and molecular sequence data. Cryptogamie Mycologie 35, 339-357.

Dai DQ, Bhat DJ, Liu JK, Chukeatirote E et al. 2012 - Bambusicola, a new genus from bamboo with asexual and sexual morphs. Cryptogamie Mycologie 33, 363-379.

Dai DQ, Phookamsak R, Wijayawardene NN, Li WJ et al. 2016 - Bambusicolous fungi. Fungal Diversity 82, 1-105.

Dai DQ, Wijayawardene NN, Bhat DJ, Chukeatirote E et al. 2014b - Pustulomyces gen. nov. accommodated in Diaporthaceae, Diaporthales, as revealed by morphology and molecular analyses. Cryptogamie Mycologie 35, 63-72.

Dai DQ, Wijayawardene NN, Bhat DJ, Chukeatirote E et al. 2014c - The phylogenetic placement of Eriosporella bambusicola sp. nov. in Capnodiales. The Scientific World Journal 35, 4149.

Daranagama DA, Camporesi E, Liu XZ, Bhat DJ et al. 2016 - Tristratiperidium microsporum gen. et sp. nov.(Xylariales) on dead leaves of Arundo plinii. Mycological Progress15, 8.

Daranagama DA, Camporesi E, Tian Q, Liu X et al. 2015 - Anthostomella is polyphyletic comprising several genera in Xylariaceae. Fungal Diversity 73, 203-238.

De Beer ZW, Duong TA, Barnes I, Wingfield BD, Wingfield MJ. 2014 - Redefining Ceratocystis and allied genera. Studies in Mycology 79, 187-219. 
De Beer ZW, Marincowitz S, Duong TA, Kim JJ et al. 2016 - Hawksworthiomyces gen. nov. (Ophiostomatales), illustrates the urgency for a decision on how to name novel taxa known only from environmental nucleic acid sequences (ENAS). Fungal Biology 120, 1323-1340.

De Gruyter J, Woudenberg JHC, Aveskamp MM, Verkley GJM et al. 2012 - Redisposition of Phoma-like anamorphs in Pleosporales. Studies in Mycology 75, 1-36.

De Silva NI, Phookamsak R, Maharachchikumbura SS, Thambugala KM et al. 2017 Monochaetia ilexae sp. nov. (Pestalotiopsidaceae) from Yunnan Province in China. Phytotaxa 291, 123-132.

Devi PT, Mathur N, Kamil D, Patel R1. 2012 - Proliferosphaera, a new genus from India. Indian Phytopathology 65, 395-398.

Diederich P, Lawrey JD, Sikaroodi M, van den Boom PPG, Ertz D. 2012 - Briancoppinsia, a new coelomycetous genus of Arthoniaceae (Arthoniales) for the lichenicolous Phoma cytospora, with a key to this and similar taxa. Fungal Diversity 52, 1-12.

Doilom M, Dissanayake AJ, Wanasinghe DN, Boonmee S et al. 2016 - Microfungi on Tectona grandis (teak) in Northern Thailand. Fungal Diversity 82, 107-182.

Dubey R, Moonnambeth AM. 2014 - Sheathnema indicum gen. et sp. nov. a new sooty mold fungus from northern Western Ghats, India. Journal of Threatened Taxa 6(12), 6549-6555.

Egidi E, de Hoog GS, Isola D, Onofri S et al. 2014 - Phylogeny and taxonomy of meristematic rock-inhabiting black fungi in the Dothideomycetes based on multi-locus phylogenies. Fungal Diversity 65, 127-165.

Ekanayaka AH, Ariyawansa HA, Hyde KD, Jones EB et al. 2017 - Discomycetes: the apothecial representatives of the phylum Ascomycota. Fungal Diversity: doi:10.1007/s13225-0170389-x.

Ertz D, Lawrey JD, Common RS, Diederich P. 2014 - Molecular data resolve a new order of Arthoniomycetes sister to the primarily lichenized Arthoniales and composed of black yeasts, lichenicolous and rock-inhabiting species. Fungal Diversity 66, 113-137.

Ertz D, Tehler A. 2011 - The phylogeny of Arthoniales (Pezizomycotina) inferred from nucLSU and RPB2 sequences. Fungal Diversity 49, 47-71.

Etayo J, Berger F. 2013 - Isthmoconidium bacidiicola, a new genus and species of lichenicolous hyphomycetes on Bacidia from Bermuda. Opuscula Philolichenum 13, 1-5.

Fu C-H, Hsieh HM, Chen C-Y, Chang TT et al. 2013 - Ophiodiaporthe cyatheae gen. et sp. nov., a diaporthalean pathogen causing a devastating wilt disease of Cyathea lepifera in Taiwan. Mycologia 105, 861-872.

Ganie AA, Azam MF, Wani AH. 2012 - Exosporodiella gen. nov. - a new coelomycetous fungus on leaves of Phoenix from India. Archives of Phytopathology and Plant Protection 45, $1582-1585$.

Grassi E, Belen Pildain M, Levin L, Carmaran C. 2014 - Studies in Diatrypaceae: the new species Eutypa microasca and investigation of ligninolytic enzyme production. Sydowia 66, 99-114.

Grum-Grzhimaylo AA, Debets AJ, van Diepeningen AD, Georgieva ML et al. 2013 -Sodiomyces alkalinus, a new holomorphic alkaliphilic ascomycete within the Plectosphaerellaceae. Persoonia 31, 147-158.

Grum-Grzhimaylo AA, Georgieva ML, Bondarenko SA, Debets AJM, Bilanenko EN. 2016 - On the diversity of fungi from soda soils. Fungal Diversity 76, 27-74.

Guatimosim E, Schwartsburd PB, Barreto RW, Crous PW. 2016 - Novel fungi from an ancient niche: cercosporoid and related sexual morphs on ferns. Persoonia 37, 106-141.

Han JG, Hosoya T, Sung GH, Shin HD. 2014 - Phylogenetic reassessment of Hyaloscyphaceae sensu lato (Helotiales, Leotiomycetes) based on multigene analyses. Fungal Biology 118, $150-167$.

Hashimoto A, Matsumura M, Hirayama K, Tanaka K. 2017 - Revision of Lophiotremataceae (Pleosporales, Dothideomycetes): Aquasubmersaceae, Cryptocoryneaceae, and Hermatomycetaceae fam. nov. Persoonia 39, 51-73 
Hashimoto A, Sato G, Matsuda T, Matsumura M et al. 2015 - Taxonomic revision of Pseudolachnea and Pseudolachnella and establishment of Neopseudolachnella and Pseudodinemasporium gen. nov. Mycologia 107, 383-408.

Healy R, Pfister DH, Rossman AY, Marvanová L et al. 2016 - Competing sexual-asexual generic names of Pezizomycetes and recommendations for use. IMA fungus 7, 285-288.

Heredia G, Ruiz R F, Arias RM, Gamboa-Angulo M et al. 2013 - Minteriella cenotigena anam. gen. \& sp. nov. from submerged plant material in Mexico. Mycological Progress 12, 271275.

Hernández Gutiérrez A. 2013 - New or rare fungi from eastern Amazonia. 1. Circinoconiopsis amazonica gen. and sp. nov. Mycotaxon 123, 107-111.

Hernández-Restrepo M, Gené J, Castañeda-Ruiz RF, Mena-Portales J et al. 2017 - Phylogeny of saprobic microfungi from Southern Europe. Studies in Mycology 86, 53-97.

Hernández-Restrepo M, Groenewald JZ, Crous PW. 2015 - Neocordana gen. nov., the causal organism of Cordana leaf spot on banana. Phytotaxa 205, 229-238.

Hernández-Restrepo M, Schumacher RK, Wingfield MJ, Ahmad I, Cai L et al. 2016 - Fungal Systematics and Evolution: FUSE 2. Sydowia 68, 193-230.

Herrera CS, Rossman AY, Samuels GJ, Chaverri P. 2013 - Pseudocosmospora, a new genus to accommodate Cosmospora viliorand related species. Mycologia 105, 1287-1305.

Heucherta B, Sanchoa LG, Braun U, Hawksworth DL. 2015 - Bicoloromyces kyffinensis: a new genus and species of lichen-inhabiting conidial fungi from $83^{\circ} 46^{\prime} \mathrm{S}$. Antarctic Science 27, 421-428.

Hongsanan S, Li YM, Liu JK, Hofmann T et al. 2014 - Revision of Asterinales. Fungal Diversity $68,1-68$.

Hongsanan S, Tian Q, Peršoh D, Zeng XY et al. 2015 - Meliolales. Fungal Diversity 74, 91-141.

Hubka V, Réblová M, Řehulka J, Selbmann L et al. 2014 - Bradymyces gen. nov.(Chaetothyriales, Trichomeriaceae), a new ascomycete genus accommodating poorly differentiated melanized fungi. Antonie van Leeuwenhoek 106, 979-992.

Hujslová M, Kubátová A, Kostovčík M, Blanchette RA et al. 2014 - Three new genera of fungi from extremely acidic soils. Mycological Progress 13, 819-831.

Hyde KD, Hongsanan S, Jeewon R, Bhat DJ et al. 2016 - Fungal diversity notes 367-490: taxonomic and phylogenetic contributions to fungal taxa. Fungal Diversity 80, 1-270.

Hyde KD, McKenzie EHC, KoKo TW. 2011 - Towards incorporating anamorphic fungi in a natural classification - checklist and notes for 2010. Mycosphere 2, 1-88.

Hyde KD, Jones EBG, Liu JK, Ariyawansa H et al. 2013 - Families of Dothideomycetes. Fungal Divers 63, 1-313.

Index Fungorum 2017 - http://www.indexfungorum.org/Names/Names. Accession Date -20 January 2017.

Ismail SI, Batzer JC, Harrington TC, Crous PW et al. 2016 - Ancestral state reconstruction infers phytopathogenic origins of sooty blotch and flyspeck fungi on apple. Mycologia 108, 292302.

Isola D, Zucconi L, Onofri S, Caneva G et al. 2016 - Extremotolerant rock inhabiting black fungi from Italian monumental sites. Fungal Diversity 76, 75-96.

Iturriaga T, Fernández R, Castañeda-Ruiz RF, Minter DW, Cruz ACR. 2013 -Scolecobeltrania, an interesting new microfungus from Venezuela. Mycotaxon 124, 143-148.

Jaklitsch WM, Fournier J, Dai DQ, Hyde KD et al. 2015 - Valsaria and the Valsariales. Fungal Diversity 73, 159-202.

Jaklitsch WM, Reblova M, Voglmayr H 2013 - Molecular systematics of Woswasia atropurpurea gen. et sp. nov. (Sordariomycetidae), a fungicolous ascomycete with globose ascospores and holoblastic conidiogenesis. Mycologia 105, 476-485.

Jaklitsch WM, Voglmayr H 2016 - Hidden diversity in Thyridaria and a new circumscription of the Thyridariaceae. Studies in Mycology 85, 35-64. 
Jayasiri SC, Hyde KD, Jones EB, Jeewon R, et al. (2017b) Taxonomy and multigene phylogenetic evaluation of novel species in Boeremia and Epicoccum with new records of Ascochyta and Didymella (Didymellaceae). Mycosphere 8, 1080-1101.

Jayasiri SC, Hyde KD, Jones EG, Ariyawansae HA et al. 2017a - A New Hysteriform Dothideomycete (Gloniaceae, Pleosporomycetidae Incertae sedis), Purpurepithecium murisporum gen. et sp. nov. on Pine Cone Scales. Cryptogamie Mycologie 38, 241-251.

Johnston PR, Seifert KA, Stone JK, Rossman AY et al. 2014 - Recommendations on generic names competing for use in Leotiomycetes (Ascomycota). IMA Fungus 5, 91-120.

Jones EG, Suetrong S, Cheng WH, Rungjindamai N et al. 2014 - An additional fungal lineage in the Hypocreomycetidae (Falcocladium species) and the taxonomic re-evaluation of Chaetosphaeria chaetosa and Swampomyces species, based on morphology, ecology and phylogeny. Cryptogamie, Mycologie 35, 119-138.

Kepler RM, Humber RA, Bischoff JF, Rehner SA. 2014 - Clarification of generic and species boundaries for Metarhizium and related fungi through multigene phylogenetics. Mycologia $106,811-829$.

Kirk P, Cannon PF, Minter DW, Stalpers JA. 2008 - Ainsworth \& Bisby's Dictionary of the Fungi. 10th edn CAB International, Wallingford, UK.

Kirk PM, Stalpers JA, Braun U, Crous PW. 2013 - A without-prejudice list of generic names of fungi for protection under the International Code of Nomenclature for algae, fungi, and plants. IMA Fungus 4, 381-443.

Klaubauf S, Tharreau D, Fournier E, Groenewald JZ et al. 2014 - Resolving the polyphyletic nature of Pyricularia (Pyriculariaceae). Studies in Mycology 79, 85-120.

Leão-Ferreira SM, Gusmão LFP, de Almeida DAC, Castañeda-Ruiz RF. 2015 Digicatenosporium polyramosum, a new hyphomycete from Brazil. Mycotaxon 130, 479-482.

Lechat C, Fournier J. 2015 - Varicosporella, a new aquatic genus in the Nectriaceae from France. Ascomycete.org. 7, 1-8.

Li GJ, Hyde KD, Zhao RL, Hongsanan S et al. 2016a - Fungal diversity notes 253-366: taxonomic and phylogenetic contributions to fungal taxa. Fungal Diversity 78, 1-237.

Li HY, Sun GY, Zhai XR, Batzer JC et al. 2012 - Dissoconiaceae associated with sooty blotch and flyspeck on fruits in China and the United States. Persoonia 28, 113-125.

Li J, Jeewon R, Luo Z, Phookamsak R et al. (2017a) Morphological characterization and DNA based taxonomy of Fusiconidium gen. nov. with two novel taxa within Melanommataceae (Pleosporales). Phytotaxa 308, 206-218.

Li J, Phookamsak R, Mapook A, Boonmee S, Bhat JD et al. 2016b - Seifertia shangrilaensis sp. nov.(Melanommataceae), a new species from Southwest China. Phytotaxa 273, 34-42.

Li JF, Bhat DJ, Phookamsak R, Mapook A et al. 2016c - Sporidesmioides thailandica gen. et sp. nov. (Dothideomycetes) from northern Thailand. Mycological Progress 15, 1169-1178.

Li JF, Phookamsak R, Jeewon R, Bhat DJ et al. (2017b) Molecular taxonomy and morphological characterization reveal new species and new host records of Torula species (Torulaceae, Pleosporales). Mycological Progress 16, 447-461.

Li WJ, Bhat DJ, Camporesi E, Tian Q, Wijayawardene NN, Dai DQ, Phookamsak R, Chomnunti P, Bahkali AH, Hyde KD. 2015 - New asexual morph taxa in Phaeosphaeriaceae. Mycosphere 6, 681-708.

Linaldeddu BT, Alves A, Phillips AJ. 2016 - Sardiniella urbana gen. et sp. nov. A new member of the Botryosphaeriaceae isolated from declining Celtis australis trees in Sardinian streetscapes. Mycosphere 7, 893-905.

Liu F, Hu DM, Cai L. 2012 - Conlarium duplumascospora gen. et. sp. nov. and Jobellisia guangdongensis sp. nov. from freshwater habitats in China. Mycologia 104, 1178-1186.

Liu JK, Hyde KD, Jones EG, Ariyawansa HA, Bhat DJ et al. 2015 - Fungal diversity notes 1-110: taxonomic and phylogenetic contributions to fungal species. Fungal Diversity 72, 1-97. 
Liu JK, Phookamsak R, Dai DQ, Tanaka K, Jones EG et al. 2014 - Roussoellaceae, a new pleosporalean family to accommodate the genera Neoroussoella gen. nov., Roussoella and Roussoellopsis. Phytotaxa 181, 1-33.

Lombard L, Houbraken J, Decock C, Samson RA et al. 2016 - Generic hyper-diversity in Stachybotryaceae. Persoonia 36,156.

Lombard L, van der Merwe NA, Groenewald JZ, Crous PW. 2014 - Lineages in Nectriaceae: reevaluating the generic status of Ilyonectria and allied genera. Phytopathologia Mediterranea $53,515-532$.

Lombard L, van der Merwe NA, Groenewald JZ, Crous PW. 2015 - Generic concepts in Nectriaceae. Studies in Mycology 80, 189-245.

Lu YZ, Boonmee S, Bhat DJ, Hyde KD et al. (2017c) Helicosporium luteosporum sp. nov. and Acanthohelicospora aurea (Tubeufiaceae, Tubeufiales) from terrestrial habitats. Phytotaxa 319, 241-253.

Lu YZ, Boonmee S, Dai DQ, Liu JK et al. 2017a - Four new species of Tubeufia (Tubeufiaceae, Tubeufiales) from Thailand. Mycological Progress 16, 403-417.

Lu YZ, Boonmee S, Liu JK, Hyde KD et al. 2017b - Novel Neoacanthostigma species from aquatic habitats. Cryptogamie, Mycologie 38, 1-22.

Lumbsch HT, Huhndorf SM. 2010 - Myconet Volume 14. Part one. Outline of Ascomycota 2009. Part Two. Notes on Ascomycete Systematics. Nos. 4751-5113. Fieldiana Life Earth Sci 1, $1-64$.

Luo J, Walsh E, Zhang N. 2015 - Toward monophyletic generic concepts in Magnaporthales: species with Harpophora asexual states. Mycologia 107, 641-646.

Luo J, Zhang N. 2013 - Magnaporthiopsis, a new genus in Magnaporthaceae (Ascomycota). Mycologia 105, 1019-1029.

Luo J, Zhuang WY. 2012 - Volutellonectria (Ascomycota, Fungi), a new genus with Volutella anamorphs. Phytotaxa 44, 1-10.

Luo ZL, Bhat DJ, Jeewon R, Boonmee S et al. 2017 - Molecular phylogeny and morphological characterization of asexual fungi (Tubeufiaceae) from freshwater habitats in yunnan, China. Cryptogamie, Mycologie 38, 1-28.

Ma J, Zhang XG, Castañeda-Ruíz RF. 2016a - Podosporiopsis, a new genus of synnematous hyphomycetes from China. Mycotaxon 131, 773-780.

Ma YR, Xia JW, Gao JM, Li XU et al. 2015 - Atrokylindriopsis, a new genus of hyphomycetes from Hainan, China, with relationship to Chaetothyriales. Mycological Progress 14, 76-80.

Ma YR, Xia JW, Gao JM, Li Z, Zhang XG. 2016b - Anacacumisporium, a New Genus Based on Morphology and Molecular Analyses from Hainan, China. Cryptogamie Mycologie 37, 45-59.

Ma YR, Xia JW, Gao JM, Li Z, Zhang XG. 2016c - Dictyoceratosporella, gen. nov., with the description of two new species collected from Hainan, China. Sydowia 68, 57-61.

Maharachchikumbura SS, Hyde KD, Jones EBG, McKenzie EHC et al. 2015 - Towards a natural classification and backbone tree for Sordariomycetes. Fungal Diversity 72, 199-301.

Maharachchikumbura SSN, Hyde KD, Groenewald JZ, Xu J, Crous PW. 2014 - Pestalotiopsis revisited. Studies in Mycology 79, 121-186.

Maharachychikumbra SSS, Hyde KD, Jones EBG, McKenzie EHC, Bhat JD, et al. 2016 - Families of Sordariomycetes. Fungal Diversity 79, 1-317.

Marin-Felix Y, Stchigel AM, Miller AN, Guarro J, Cano-Lira JF. 2015 - A re-evaluation of the genus Myceliopthora (Sordariales, Ascomycota): its segregation into four genera and description of Corynascus fumimontanus sp. nov. Mycologia 107, 619-632.

Marvanová L, Laichmanová M. 2014 -Campylospora leptosome sp. nov. and characteristics of Campylospora spp., based on morphology and on ITS sequences. Mycosphere 5, 245-261.

Mel'nik VA, Crous PW. 2014 - Braunomyces dictyosporus gen. et sp. nov. from Vietnam. IMA Fungus 5, 1-5. 
Monteiro JS, Castañeda-Ruiz RF, Gusmão LFP. 2014a - Helicodochium new microfungus from submerged wood from Brazil. Mycotaxon 127, 5-9.

Monteiro JS, Conceição LB, Marques MF, Gusmão LF, Castañeda-Ruiz RF. 2016a Dictyoaquaphila appendiculata gen. \& sp. nov. from submerged wood from Brazil. Mycotaxon 131, 177-183.

Monteiro JS, Gusmão LF, Castañeda-Ruiz RF. 2014b - Two new microfungi from Brazilian Amazon Forest: Atrogeniculata submersa and Nigrolentilocus amazonicus. Mycotaxon 127, 39-45.

Monteiro JS, Gusmão LF, Castañeda-Ruiz RF. 2015 - Brachycephala exotica, a new hyphomycete from Brazil. Mycotaxon 130, 489-493.

Monteiro JS, Gusmão LF, Castañeda-Ruiz RF. 2016b - Anacoronospora diversiseptata gen. \& sp. nov. from Brazil. Mycotaxon 131, 185-192.

Nascimento MM, Selbmann L, Sharifynia S, Al-Hatmi AM et al. 2016 - Arthrocladium, an unexpected human opportunist in Trichomeriaceae (Chaetothyriales). Fungal Biology 120, 207-218.

Norphanphoun C, Hongsanan S, Doilom M, Bhat DJ et al. 2016 - Lamproconiaceae fam. nov. to accommodate Lamproconium desmazieri. Phytotaxa 270, 89-102.

Otálora MA, Jørgensen PM, Wedin M. 2014 - A revised generic classification of the jelly lichens, Collemataceae. Fungal Diversity 64, 275-293.

Pärtel K, Baral HO, Tamm H, Põldmaa K. 2016 - Evidence for the polyphyly of Encoelia and Encoelioideae with reconsideration of respective families in Leotiomycetes. Fungal Diversity 82, 183-219.

Pažoutová S, Šrůtka P, Holuša J, Chudíčková M et al. 2012 - Liberomyces gen. nov. with two new species of endophytic coelomycetes from broadleaf trees. Mycologia 104, 198-210.

Perdomo H, García D, Gené J, Cano J et al. 2013 - Phialemoniopsis, a new genus of Sordariomycetes, and new species of Phialemonium and Lecythophora. Mycologia 105, 398-421.

Perera RH, Maharachchikumbura SS, Bahkali AH, Camporesi E et al. 2016 - Sexual morph of Seimatosporium cornii found on Cornus sanguinea in Italy. Phytotaxa 257, 51-60.

Phillips AJL, Alves A, Abdollahzadeh J, Slippers B et al. 2013 - The Botryosphaeriaceae: genera and species known from culture. Studies in Mycology 76, 51-167.

Phookamsak R, Liu J-K, McKenzie EHC, Manamgoda DS et al. 2014 - Revision of Phaeosphaeriaceae. Fungal Diversity 68, 159-238.

Phukhamsakda C, Ariyawansa HA, Phillips AJ, Wanasinghe DN et al. 2016a - Additions to Sporormiaceae: Introducing two novel genera, Sparticola and Forliomyces, from Spartium. Cryptogamie Mycologie 37, 75-97.

Phukhamsakda C, Ariyawansa HA, Phookamsak R, Chomnunti P et al. 2015 - Muriphaeosphaeria galatellae gen. et sp. nov. in Phaeosphaeriaceae (Pleosporales). Phytotaxa 227, 55-65.

Phukhamsakda C, Hongsanan S, Ryberg M, Ariyawansa HA et al. (2016b) The evolution of Massarineae with Longipedicellataceae fam. nov. Mycosphere 7, 1713-1731.

Prabhugaonkar A, Bhat DJ. 2011 - New record of Megacapitula villosa and Paradictyoarthrinium diffractum from India. Mycosphere 2, 463-467.

Pratibha J. 2013 - Jayarambhatia rhizophorae gen. et sp. nov., an asexually reproducing fungus from Goa, India. Mycotaxon 125, 139-144.

Punithalingam E, Spooner BM. 2011 - A new fungicolous Scolecobasidium (hyphomycetes) and Caducirostrum gen. nov. (coelomycetes) from leaf litter in the UK and Italy. Kew Bulletin 66, 309-324.

Quaedvlieg W, Binder M, Groenewald JZ, Summerell BA et al. 2014 - Introducing the consolidated species concept to resolve species in the Teratosphaeriaceae. Persoonia 33, 140.

Quaedvlieg W, Verkley GJM, Shin H-D, Barreto RW et al. 2013 - Sizing up Septoria. Studies in Mycology 75, 307-390. 
Rajeshkumar KC, Sharma R. 2013 - Tamhinispora a new genus belongs to family Tubeufiaceae from the Western Ghats, India based on morphology and phylogenetic analysis. Mycosphere 4, 165-174.

Rämä T, Mathiassen GH, Kauserud H. 2014 - Marine fungi new to Norway, with an outlook to the overall diversity. Agarica 35, 35-47.

Réblová M 2013 - Two taxonomic novelties in the Sordariomycetidae: Ceratolenta caudata gen. et sp. nov. and Platytrachelon abietis gen. et comb. nov. for Ceratosphaeria abietis. Mycologia 105, 462-475.

Réblová M, Miller AN, Rossman AY, Seifert KA et al. 2016a - Recommendations for competing sexual-asexually typified generic names in Sordariomycetes (except Diaporthales, Hypocreales, and Magnaporthales). IMA fungus 7, 131-153.

Réblová M, Seifert KA, Fournier J, Stepánek V. 2012 - Phylogenetic classification of Pleurothecium and Pleurotheciella gen. nov. and its dactylaria-like anamorph (Sordariomycetes) based on nuclear ribosomal and protein-coding genes. Mycologia 104, 1299-1314.

Réblová M, Seifert KA, Fournier J, Štěpánek V. 2016b - Newly recognised lineages of perithecial ascomycetes: the new orders Conioscyphales and Pleurotheciales. Persoonia 37, 57-81.

Réblová M, Štěpánek V, Schumacher RK 2014 - Xylochrysis lucidagen. et sp. nov., a new lignicolous ascomycete (Sordariomycetidae) with holoblastic conidiogenesis. Mycologia $106,564-572$.

Réblová M, Untereiner WA, Réblová K. 2013 - Novel Evolutionary Lineages Revealed in the Chaetothyriales (Fungi) Based on multigene phylogenetic analyses and comparison of its secondary structure. PLoS ONE 8: doi: 10.1371/annotation/acfe0686-dd9d-4673-8cc9$3 b b 9 f 457 a 8 c 4$.

Ren Sh.C, Ma J, Ma LG, Zhang YD, Zhang XG. 2012 - Sativumoides and Cladosporiopsis, two new genera of hyphomycetes from China. Mycological Progress 11, 443-448.

Rossman AY, Adams GC, Cannon PF, Castlebury LA et al. 2015a - Recommendations of generic names in Diaporthales competing for protection or use. IMA fungus 6, 145-154.

Rossman AY, Allen WC, Braun U, Castlebury LA et al. 2016 - Overlooked competing asexual and sexually typified generic names of Ascomycota with recommendations for their use or protection. IMA fungus 7, 289-308.

Rossman AY, Crous PW, Hyde KD, Hawksworth DL et al. 2015b - Recommended names for pleomorphic genera in Dothideomycetes. IMA fungus 6, 507-523.

Rossman AY, Seifert KA, Samuels GJ, Minnis AM et al. 2013 - Genera in Bionectriaceae, Hypocreaceae, and Nectriaceae (Hypocreales) proposed for acceptance or rejection. IMA fungus $4,41-51$.

Samerpitak K, Van der Linde E, Choi HJ, van den Ende AG, Machouart M et al. 2014 - Taxonomy of Ochroconis, genus including opportunistic pathogens on humans and animals. Fungal Diversity 65, 89-126.

Sandoval Denis M, Gené J, Sutton DA, Cano-Lira JF et al. 2016 - Redefining Microascus, Scopulariopsis and allied genera. Persoonia 36,1-36.

Sanoamuang N, Jitjak W, Rodtong S, Whalley JS. 2013 - Gelatinomyces siamensis gen. sp. nov. (Ascomycota, Leotiomycetes, incertae sedis) on bamboo in Thailand. IMA fungus 4, 71-80.

Santa-Izabel TS, Almeida DAC, Monteiro JS, Marques MFO et al. 2015 - Anaexserticlava caatingae, a new conidial fungus from the semi-arid Caatinga biome of Brazil. Mycotaxon 130, 445-449.

Santa-Izabel TS, Cruz ACR, Gusmão LFP. 2013 - Conidial fungi from the semi - arid Caatinga biome of Brazil. Ellisembiopsis gen. nov., new variety of Sporidesmiella and some notes of Sporidesmium complex. Mycosphere 4, 156-163.

Seifert K, Jones MG, Gams W, Kendrick B. 2011 - The genera of hyphomycetes. CBS biodiversity series no. 9:1-997. CBS-KNAW Fungal Biodiversity Centre, Utrecht. 
Selbmann L, Isola D, Egidi E, Zucconi L et al. 2014 - Mountain tips as reservoirs for new rockfungal entities: Saxomyces gen. nov. and four new species from the Alps. Fungal Diversity 65, 167-182.

Senanayake IC, Maharachchikumbura SSN, Hyde KD, Bhat JD et al. 2015 - Towards unraveling relationships in Xylariomycetidae (Sordariomycetes). Fungal Diversity 73, 73-144.

Senanayake IC, Maharachchikumbura SSN, Jeewon R, Promputtha I et al. 2017 Morphophylogenetic study of Sydowiellaceae reveals several new genera. Mycosphere 8, 172-217.

Sharma R, Sharma R, Crous PW. 2015 - Matsushimamyces, a new genus of keratinophilic fungi from soil in central India. IMA Fungus 6, 337-343.

Sigler L, Hambleton S, Paré JA. 2013 - Molecular characterization of reptile pathogens currently known as members of the Chrysosporium anamorph of Nannizziopsis vriesii (CANV) complex and relationship with some human-associated isolates. Journal of Clinical Microbiology 51, 3338-3357.

Silva CR, Castañeda Ruiz RF, Gusmão LFP. 2014 - Anacraspedodidymum, a new genus from submerged wood in Brazil. Mycotaxon 128, 11-15.

Silva CR, Gusmão LFP, Castañeda-Ruiz RF 2015 - Two new conidial fungi from Chapada Diamantina, Brazil. Mycotaxon 130, 437-443.

Silva M da, Castañeda Ruiz RF, Pereira OL, Barreto RW. 2012 - Alveariospora, a new anamorphic genus from trichomes of Dimorphandra mollis in Brazil. Mycotaxon 119, 109-116.

Silva SS, Gusmão LF, Castañeda-Ruiz RF 2016 - Arthromoniliphora araucariae gen. \& sp. nov. from Brazilian pine. Mycotaxon 131, 821-826.

Sri-indrasutdhi V, Tsui CKM, Chuaseeharonnachai C, Yamaguchi K et al. 2015 - Helicocentralis hyalina gen. et sp. nov., an aero-aquatic helicosporous fungus (Leotiomycetes, Ascomycota) in Thailand Mycological Progress 14, 80-92.

Su HY, Hyde KD, Maharachchikumbura SSN, Ariyawansa HA et al. 2016 - The families Distoseptisporaceae fam. nov., Kirschsteiniotheliaceae, Sporormiaceae and Torulaceae, with new species from freshwater in Yunnan Province, China. Fungal Diversity 80, 375409.

Su L, Guo L, Hao Y, Xiang M, Cai L, Liu X. 2015 - Rupestriomyces and Spissiomyces, two new genera of rock-inhabiting fungi from China. Mycologia 107, 831-844.

Suetrong S, Klaysuban A, Sakayaroj J, Preedanon S et al. 2015 - Tirisporellaceae, a new family in the order Diaporthales (Sordariomycetes, Ascomycota). Cryptogamie Mycologie 36, 319-330.

Suetrong S, Rungjindamai N, Sommai S, Rung-Areerate P et al. 2014 - Wiesneriomyces a new lineage of Dothideomycetes (Ascomycota) basal to Tubeufiales. Phytotaxa 176, 283-297.

Tan YP, Madrid H, Crous PW, Shivas RG. 2014 - Johnalcornia gen. et. comb. nov., and new combinations and synonymies in Bipolaris and Curvularia. 43, 589-603.

Tanaka K, Hirayama K, Yonezawa H, Sato G et al. 2015 - Revision of the Massarineae (Pleosporales, Dothideomycetes). Studies in Mycology 82, 75-136.

Thambugala KM, Ariyawansa HA, Li YM, Boonmee S et al. 2014 - Dothideales. Fungal Diversity $68,105-158$.

Thambugala KM, Chunfang Y, Camporesi E, Bahkali AH, Liu ZY et al. 2015a Pseudodidymosphaeria gen. nov. in Massarinaceae. Phytotaxa 231, 271-282.

Thambugala KM, Daranagama DA, Phillips AJ, Bulgakov TS et al. 2016 - Microfungi on Tamarix. Fungal Diversity 82, 239-306.

Thambugala KM, Hyde KD, Tanaka K, Tian Q et al. 2015b - Towards a natural classification and backbone tree for Lophiostomataceae, Floricolaceae and Amorosiaceae fam. nov. Fungal Diversity 74, 199-266.

Thambugala KM, Wanasinghe DN, Phillips AJL, et al. 2017 - Mycosphere notes 1-50: Grass (Poaceae) inhabiting Dothideomycetes. Mycosphere 8, 697-796. 
Tian Q, Doilom M, Luo ZL, Chomnunti P et al. 2016b - Introducing Melanoctona tectonae gen. et sp. nov. and Minimelanolocus yunnanensis sp. nov. (Herpotrichiellaceae, Chaetothyriales). Cryptogamie Mycologie 37, 477-492.

Tian Q, Liu JK, Hyde KD, Wanasinghe DN et al. 2015 - Phylogenetic relationships and morphological reappraisal of Melanommataceae (Pleosporales). Fungal Diversity 74, 267324.

Tibpromma S, Hyde KD, Jeewon R, Maharachchikumbura SSN et al. 2017 - Fungal diversity notes 491-602: taxonomic and phylogenetic contributions to fungal taxa. Fungal Diversity 83, 1261

Trakunyingcharoen T, Lombard L, Groenewald JZ, Cheewangkoon R et al. 2014 - Mycoparasitic species of Sphaerellopsis, and allied lichenicolous and other genera. IMA fungus 5, 391-414.

Vázquez-Campos X, Kinsela AS, Waite TD, Collins RN, Neilan BA. 2014 - Fodinomyces uranophilus gen. nov. sp. nov. and Coniochaeta fodinicola sp. nov., two uranium mine inhabiting Ascomycota fungi from northern Australia. Mycologia 106, 1073-1089.

Verkley GJM, Dukik K, Renfurm R, Göker M, Stielow JB. 2014 - Novel genera and species of coniothyrium-like fungi in Montagnulaceae (Ascomycota). Persoonia 32, 25-51.

Verkley GJM, Quaedvlieg W, Shin HD, Crous PW. 2013 - A new approach to species delimitation in Septoria. Studies in Mycology 75, 213-305.

Videira SI, Groenewald JZ, Braun U, Shin HD, Crous PW. 2016 - All that glitters is not Ramularia. Studies in Mycology 83, 49-163.

Voglmayr H, Castlebury LA, Jaklitsch WM 2017 - Juglanconis gen. nov. on Juglandaceae, and the new family Juglanconidaceae (Diaporthales). Persoonia 38, 136-155.

Voglmayr H, Gardiennet A, Jaklitsch WM. 2016 - Asterodiscus and Stigmatodiscus, two new apothecial dothideomycete genera and the new order Stigmatodiscales. Fungal Diversity 80, 271-284.

Voglmayr H, Jaklitsch WM 2017 - Corynespora, Exosporium and Helminthosporium revisited New species and generic reclassification. Studies in Mycology 87, 43-76.

Wäli PP, Huhtinen S, Pino-Bodas R, Stenroos S. 2014 - Three common bryophilous fungi with meristematic anamorphs and phylogenetic alliance to Teratosphaeriaceae, Capnodiales. Fungal Biology 118, 956-969.

Walsh E, Luo J, Naik A, Preteroti T, Zhang N. 2015 - Barrenia, a new genus associated with roots of switchgrass and pine in the oligotrophic pine barrens. Fungal Biology 119, 1216-1225.

Walsh E, Luo J, Zhang N. 2014 - Acidomelania panicicola gen. et sp. nov. from switchgrass roots in acidic New Jersey pine barrens. Mycologia 106, 856-864.

Wanasinghe DN, Jones EBG, Camporesi E, Boonmee S et al. 2014 - An exciting novel member of Lentitheciaceae in Italy from Clematis vitalba. Cryptogamie Mycologie 35, 323-337.

Wanasinghe DN, Jones EG, Camporesi E, Mortimer PE, Xu J et al. 2015 - The genus Murispora. Cryptogamie Mycologie 36, 419-448.

Wanasinghe DN, Phookamsak R, Jeewon R, Li WJ et al. 2017 - Fenestellaceae with descriptions of new Fenestella species and Neocucurbitaria gen. nov. Mycosphere 8, 397-414.

Wen TC, Xiao YP, Han YF, Huang SK et al. 2017 - Multigene phylogeny and morphology reveal that the Chinese medicinal mushroom 'Cordyceps gunnii' is Metacordyceps neogunnii sp. nov. Phytotaxa 302, 27-39.

Wendt L, Sir EB, Kuhnert E, Heitkämper S et al. 2017 - Resurrection and emendation of the Hypoxylaceae, recognised from a multigene phylogeny of the Xylariales. Mycological Progress DOI 10.1007/s11557-017-1311-3.

Wijayawardene DNN, McKenzie EHC, Hyde KD. 2012 - Towards incorporating anamorphic fungi in a natural classification-checklist and notes for 2011. Mycosphere 3, 157-228.

Wijayawardene NN, Bhat DJ, Hyde KD, Camporesi E et al. 2014a - Camarosporium sensu stricto in Pleosporinae, Pleosporales with two new species. Phytotaxa 183, 16-26. 
Wijayawardene NN, Camporesi E, Song Y, Dai DQ et al. 2013 - Multigene analyses reveal taxonomic placement of Scolicosporium minkeviciusiiin Phaeosphaeriacea (Pleosporales). Cryptogam Mycol 34(4):357-366.

Wijayawardene NN, Crous PW, Kirk PM, Hawksworth DL et al. 2014b - Naming and outline of Dothideomycetes-2014 including proposals for the protection or suppression of generic names. Fungal Diversity 69, 1-55.

Wijayawardene NN, Hyde KD, Bhat DJ, Camporesi E et al. 2014c - Camarosporium-like species are polyphyletic in Pleosporales; introducing Paracamarosporium and Pseudocamarosporium gen. nov. in Montagnulaceae. Cryptog Mycol 35:177-219.

Wijayawardene NN, Hyde KD, Camporesi E, Bhat DJ et al. 2015 - Additions to brown spored coelomycetous taxa in Massarinae, Pleosporales: introducing Phragmocamarosporium gen. nov. and Suttonomyces gen. nov. Cryptogamie Mycologie 36, 213-224.

Wijayawardene NN, Hyde KD, Rajeshkumar KC, Hawksworth DL et al. 2017 - Notes for genera Ascomycota. Fungal Diversity (in press).

Wijayawardene NN, Hyde KD, Wanasinghe DN, Papizadeh M et al. 2016 - Taxonomy and phylogeny of dematiaceous coelomycetes. Fungal Diversity Fungal Diversity 77, 1-316.

Woudenberg JHC, Groenewald JZ, Binder M, Crous PW. 2013 - Alternaria redefined. Studies in Mycology 75, 171-212.

Xia JW, Ma YR, Gao JM, Li Z et al. 2016 - Sympodiosynnema, a new genus of dematiaceous hyphomycetes from southern China. Mycotaxon 131, 45-48.

Xia JW, Ma YR, Gao JM, Zhang XG, Li Z. 2016 - Atrosynnema, a new hyphomycete from dead branches in China. Mycotaxon 131, 287-290.

Yang J, Maharachchikumbura SS, Bhat DJ, Bahkali AH et al. 2016 - Aquapteridospora lignicola gen. et sp. nov., a new hyphomycetous taxon (Sordariomycetes) from wood submerged in a freshwater stream. Cryptogamie Mycologie 36, 469-478.

Yang J, Maharachchikumbura SS, Liu JK, Hyde KD et al. 2017 - Pseudostanjehughesia aquitropica gen. et sp. nov. and Sporidesmium sensu lato species from freshwater habitats. Mycological Progress https://doi.org/10.1007/s11557-017-1339-4.

Zawadneak MA, Pimentel IC, Robl D, Dalzoto P et al. 2015 - Paecilomyces niveus Stolk \& Samson, 1971 (Ascomycota: Thermoascaceae) as a pathogen of Nasonovia ribisnigri (Mosley, 1841) (Hemiptera, Aphididae) in Brazil. Brazilian Journal of Biology 75, $158-162$.

Zhang H, Dong W, Hyde KD, Bahkali AH et al. 2016 - Molecular data shows Didymella aptrootii is a new genus in Bambusicolaceae. Phytotaxa 247, 99-108.

Zhang H, Hyde KD, Mckenzie EH, Bahkali AH et al. 2012a - Sequence data reveals phylogenetic affinities of Acrocalymma aquatica sp. nov., Aquasubmersa mircensis gen. et sp. nov. and Clohesyomyces aquaticus (freshwater coelomycetes). Cryptogamie Mycologie 33, 333-346.

Zhang K, Ma LG, Ma J. Castañeda-Ruiz RF. 2014 - Xiuguozhangia, a new genus of microfungi to accommodate five Piricaudiopsis species. Mycotaxon 128, 131-135.

Zhang N, Luo J, Rossman AY, Aoki T, Chuma I et al. 2016 - Generic names in Magnaporthales. IMA fungus 7, 155-159.

Zhang YD, Ma J, Ma LG, Zhang XG. 2012b - Parablastocatena tetracerae gen. et sp. nov. and Corynesporella licualae sp. nov. from Hainan, China. Mycoscience 53, 381-385.

Zhao KN, Yin GH, Zhao GZ, Cao AX. 2013 - Embryonispora, a new genus of hyphomycetes from China. Mycotaxon 126, 77-81.

Zhurbenko MP, Braun U. 2013 - Ameroconium cladoniae gen. et sp. nov. and Phoma psoromatis sp. nov., new anamorphic lichenicolous fungi from the Holarctic. The Lichenologist 45, 583-591.

Zhurbenko MP, Himelbrant DE, Kuznetsova ES, Stepanchikova IS. 2012 - Lichenicolous fungi from the Kamchatka Peninsula, Russia. The Bryologist 115, 295-312. 
Zhurbenko MP, Pino-Bodas RA. 2017 - A revision of lichenicolous fungi growing on Cladonia, mainly from the Northern Hemisphere, with a worldwide key to the known species. Opuscula Philolichenum 16, 188-266. 